\title{
The Limit of Strength and Toughness of Steel
}

\author{
Zhen Guo \\ $\mathrm{Ph}$. D. Thesis \\ Department of Materials Science and Mineral Engineering \\ University of California, Berkeley \\ and \\ Materials Science Division \\ Ernest Orlando Lawrence Berkeley National Laboratory \\ University of California \\ Berkeley, CA 94720
}

December, 2001

This work was supported by the Director, Office of Science, Office of Basic Energy Science, Materials

Science Division, of the U. S. Department of Energy under Contract of No. DE-AC03-76SF00098 


\title{
The Limit of Strength and Toughness of Steel
}

by

\author{
Zhen Guo \\ B. E. (Tsinghua University) 1994 \\ M. S. (University of Michigan) 1998
}

A dissertation submitted in partial satisfaction of the requirements for the degree of

Doctor of Philosophy

in

Engineering - Materials Science and Engineering

in the

GRADUATE DIVISION

of the

UNIVERSITY OF CALIFORNIA, BERKELEY

Committee in charge:

Professor John Williams Morris, Jr., Chair

Professor Daryl C. Chrzan

Professor Zi Q. Qiu

Fall, 2001 
The dissertation of Zhen Guo is approved:

\begin{tabular}{lr}
\hline Chair & Date \\
\hline & Date \\
\hline & Date
\end{tabular}

University of California, Berkeley

Fall, 2001 


\section{The Limit of Strength and Toughness of Steel}

Copyright (C) 2001

by

Zhen Guo

The U.S. Department of Energy has right to use this document of any purpose whatsoever including the right to reproduce all or any part thereof. 


\author{
Abstract \\ The Limit of Strength and Toughness in Steel \\ by \\ Zhen Guo \\ Doctor of Philosophy in Materials Science and Engineering \\ University of California, Berkeley
}

Professor John Williams Morris, Jr., Chair

The ideal structural steel combines high strength with high fracture toughness. This dissertation discusses the governing principles of strength and toughness, along with the approaches that can be used to improve these properties and the inherent limits to how strong and tough a steel can be.

Chapter I provides the background on fracture mechanics and describes the factors that affect cleavage fracture from atomic, microstructural and macroscopic levels. From such considerations, the inherent resistance to cleavage fracture is apparent.

Chapter II discusses the ideal fracture strength and the critical fracture strength in high strength steels. Recent $a b$ intio computations predict an ideal cleavage strength of Fe near $14 \mathrm{GPa}$ on $\{100\}$ planes, indicating that the normal fracture mode of high strength steel, 
characterized by a yield strength greater than about $4 \mathrm{GPa}$ is brittle. A preliminary study of the effect of alloying with $\mathrm{Ni}$ shows a trend of decreasing brittle behavior with increasing Ni content. More sophisticated methods to study this effect are proposed.

Chapter III examines the practical methods for achieving properties that approach the limits of strength and toughness of steel. The discussion focuses on grain refinement through thermal treatments in lath martensitic steels. The objective is to limit the crystallographic coherence length for transgranular cleavage propagation. A new method is presented for directly disrupting the crystallographic orientation alignment within a martensitic packet, and thereby achieving submicron effective grain size. This has been accomplished in AerMet 100 and 9Ni steel by an alternate "intercritical annealing" (L) and "reversion" $(\mathrm{Q})$ treatment. An "L" treatment creates a dual-phase microstructure containing fine parallel laths with different alloy contents. Subsequent austenite reversion ("Q" treatment) leads to a two-step martensitic transformation during cooling, disrupting the laths within a packet and generating a large misorientation between the adjacent laths.

Chapter IV explains why this grain refinement can be accomplished, as described by martensitic transformation theory. It is shown that all KS and NW relationships can be divided into three Bain variant groups based on their transformation strain matrix. Only the variants from different Bain groups will have a large angle of misorientation. Furthermore, multiple variants from different Bain groups might appear if the geometric constraint prevents the relaxation of transformation strain. 
To my wife, Nan, and my parents 


\section{Acknowledgments}

First of all, I deeply appreciate the fully trust, freedom and guidance that my advisor, J. W. Morris, Jr. has given me during my graduate career in Berkeley. Without all the discussions, brainstorms and lunches we had together, it is impossible for me to finish my Doctor degree on martensitic steels, a topic that I knew very little about before. I also enjoyed the challenging questions posed by my qualifying exam committee: R. O. Ritchie (Chair), D. C. Chrzan, T. D. Sands and Z. Q. Qiu. I am thankful for the helpful suggestion and criticism offered by my dissertation committee: J. W. Morris, Jr., D. C. Chrzan and Z. Q. Qiu.

I am grateful for the support provided by the people outside and within our group. Chip Flor and James $\mathrm{Wu}$ helped me to prepare the materials and samples. Eric Stach and Chuck Echer gave me a lot of help on Transmission Electron Microscope. Jin taught me how to use the equipment in our Lab and Chris is the "to-go" people in the "imaginary world (computer and theory)". Dave Mitlin, Andy and Hogeon, thank you so much for all the discussions and helps among our "new generation grads" across 38 degree line, the wall between 337 and 336. It has been a great pleasure to work with and learn from Carol, Tae-Kyu, Dave Clatterbuck, Brian, Erica, Miao, Dr. Kim, Dr. Lee, Dr. Liu. I am also very thankful to Andy and Erica, who reviewed the draft of this dissertation and gave me a lot of technical suggestions as well as corrected me numerous English mistakes. 
Finally, my wife Henan (Anne) deserves a special appreciation for the continued encouragement and support she has entrusted me, for all the happiness, difficulties and dreams we share together in these years. I also thank my Mom and Dad for their love and care, for they always say that I am their greatest achievement.

This work was funded by the Director, Office of Energy Research, Office of Basic Energy Science, Materials Science Division of the U.S. Department of Energy, under contract No. DE-AC03-76SF00098. 
Contents

List of Figures viii

List of Tables $\quad$ xvi

Chapter I General Introduction 1

1.1 Fracture Modes of Steels 3

1.2 Transgranular Cleavage Fracture 7

1.3 General Consideration and Approaches 10

$\begin{array}{lll}\text { Chapter II Fracture Strength Criterion } & 14\end{array}$

2.1 Ideal Fracture Strength 14

2.1.1 Classic model for ideal fracture strength 14

2.1.2 The strain at elastic instability 16

2.2 Apparent Fracture Strength and Ductile - Brittle Fracture

$\begin{array}{ll}\text { Transitions } & 20\end{array}$

2.2.1 In flaw-free single crystal Fe 20

2.2.2 In pure iron and plain steels with defects 21

2.2.3 In high and ultrahigh strength steels 23

2.3 Effect of Alloying Chemistry on Fracture Strength 25

$2.4 \quad$ Future Work 28 
3.1 Introduction 31

3.1.1 Why grain refinement 31

3.1.2 Approaches to grain refinement 32

3.1.3 Effective grain size in lath martensitic steels 33

3.1.4 Thermal mechanisms of grain refinement in lath martensitic steel 36

3.2 Experimental Procedures 38

3.3 Microstructural Evolutions 41

3.3.1 Normalization (N) treatment 41

3.3.2 Reversion (Q) treatment 41

3.3.3 Intercritical annealing (L) treatment 46

3.3.4 Intercritical annealing and reversion $(L Q)$ treatment 51

3.3.5 Microstructure observation in 9Ni steel 60

3.4 Mechanical Properties 63

$\begin{array}{lll}3.5 & \text { Conclusions } & 68\end{array}$

Chapter IV Martensitic Transformation Theory

for Grain Refinement 69

$\begin{array}{lll}4.1 & \text { Introduction } & 69\end{array}$

4.1.1 Bain correspondence and distortion $\quad 70$

4.1.2 Shear Mechanism models 71

4.1.3 Double shear mechanism - crystallographic theory 72 
4.1.4 Linear elasticity theory of martensitic transformation

4.2 Calculations of Elastic Strain

4.2.1 Deformation and strain matrices for $N$ - $W$ relationship using FCC coordinates

4.2.2 Deformation and strain matrices for $K-S$ relationship using FCC coordinates

4.2.3 Deformation and strain matrices using BCC coordinates

4.3 Invariant Plane Strain

4.3.1 Eshelby Circle for elastic inclusion problem

4.3.2 Stress and Strain field calculations

4.3.3 Necessity of invariant plane strain condition

4.3.4 Achievement of invariant strain condition

4.4 Crystallographic Relationship and Grain Refinement

4.4.1 Grouping $K-S$ and $N-W$ relations into three Bain variant groups

4.4.2 Orientation relations between different and within same Bain variant groups

4.4.3 Four-color theorem and grain refinement of ferrite

4.5 Elastic Theory and Thermal Mechanisms of Grain

Refinement 
4.6 Revisiting on the Meaning of Effective Grain Size and Grain

Refinement

4.7 Conclusions 


\section{List of Figures}

Fig. 1-1 Mechanical properties of typical high and ultrahigh strength steels

Fig. 1-2 Three typical failure modes of high and ultrahigh strength steels

Fig. 1-3 (a) Typical ductile - brittle transition in high and ultrahigh strength steels and (b) a schematic drawing of Yoffee Diagram as an explanation

Fig. 1-4 Schematic illustrations of factors in (a) atomic, (b) microscopic and (c) macroscopic level that affect brittle transgranular fracture behavior.

Fig. 2-1 Potential energy and force as a function of atomic separation

Fig. 2-2 The Bain strain connecting the $\mathrm{BCC}$ and $\mathrm{FCC}$ structure. If $\mathrm{BCC}$ is pulled in tension along [001] direction while contracting along [100] and [010], it generates a FCC crystal as shown.

Fig. 2-3 (a) The stress-strain curve of AerMet 100 at 77K. (b) Elastic-Plastic FEM solutions for the maximum tensile stress as a function of the applied stress intensity. The value of $\mathrm{K}_{\mathrm{IC}}$ measured in a specific test is indicated. 
Fig. 2-4 The influence of Ni on the ideal cleavage and shear strength of Fe. The lower dash line shows the resolved shear stress on $<111>\{112\}$ generated by cleavage stress normal to $\{100\}$

Fig. 3-1 The microstructure of a lath martensitic steel. (a) Optical microstructure of lath martensitic Steel; (b) Illustration of subdivided packet within a prior austenite grain; (c) dislocated lath martensite within a packet showing alignment of laths of less than $0.5 \mathrm{~mm}$ thickness; (d) The diffraction pattern shows the laths are almost identical in crystallographic orientation

Fig. 3-2 Scanning electron micrograph of etched cross-section of lath martensitic steel broken below $\mathrm{T}_{\mathrm{B}}$. The cleavage cracks follow common $\{100\}$ planes across parallel laths in a packet, indicating that the effective grain size is the packet size.

Fig. 3-3 Schematic phase diagram of the five heat treatments used in this research.

Fig. 3-4 TEM microstructure of AerMet 100 in a standard QT condition (1158, 1hr, oil quenched; 77K $1 \mathrm{hr}$ for chilling; 755K, 5hrs for tempering). Local diffraction pattern indicates two sets of diffraction pattern (variants) in a martensite packet. 
Fig. 3-5 Optical and TEM Microstructure of AerMet 100 after the $\mathrm{N}$ treatment (1423K, 18 hours for normalization; 77K, 1 hour for chilling), showing large prior austenite grains. Diffraction patterns taken inside the packet exhibit only one variant, indicating well-aligned martensite laths.

Fig. 3-6 Optical and TEM microstructure of AerMet 100 in the NQT condition (1423K, 18hrs for normalization; $1158 \mathrm{~K} 1 \mathrm{hr}$ oil quenched; $77 \mathrm{~K} 1 \mathrm{hr}$ for chilling; $755 \mathrm{~K}$, 5hrs for tempering), showing prior austenite grains have been significantly refined. However, the diffraction pattern taken within a packet still indicates that good crystallographic alignment remains in martensite packets.

Fig. 3-7 Optical microstructure of AerMet 100 after heat-treated at 1423K, $18 \mathrm{hrs}$ for normalization; and $1158 \mathrm{~K}$ for (a) 1 minute and (b) 2 minutes, indicating that recrystallization occurs through nucleation and growth of new austenite grains from grain boundaries in most cases and sometimes inside grains.

Fig. 3-8 Optical and TEM microstructure of AerMet 100 in NL condition (1423K 18hrs for normalization; 950K, $1 \mathrm{hr}$ for intercritical annealing), showing a lamellar pattern alternating between heavily dislocated fresh martensite laths and tempered martensite laths. However, diffraction pattern exhibits only one variant within the packet. 
Fig. 3-9 EDAX results on individual lath of AerMet 100 in NL condition (1423K $18 \mathrm{hrs}$ for normalization; 950K, $1 \mathrm{hr}$ for intercritical annealing) revealed that solute distribute alternatively in the adjacent laths in a lamellar pattern. However, $\mathrm{Ni}$ and $\mathrm{Co}$ have opposite trends since $\mathrm{Ni}$ is austenite stabilizer and Co favors ferrite.

Fig. 3-10 Schematic Illustrations of (a) austenite nucleation at lath boundaries, (b) austenite growth along lath boundary to form a thin layer of precipitated austenite and (c) adjacent martensite laths have been tempered during intercritical annealing treatment (L), resulting in a lamellar structure of dual phase steel.

Fig. 3-11 TEM microstructure of AerMet 100 in the NLQT condition (1423K, 18hrs for normalization; 950K, $1 \mathrm{hr}$ for intercritical annealing; $1158 \mathrm{~K}, 1 \mathrm{hr}$, oil quenched; $77 \mathrm{~K}$, $1 \mathrm{hr}$ for chilling, $755 \mathrm{~K}$, 5hrs for tempering). Local diffraction pattern indicates that adjacent laths have different variants in the same martensite packet.

Fig. 3-12 Crystallographic illustration of K-S and N-W orientation relationships between martensite and austenite, indicating that there are small misfit but large misorientation on the boundaries between two adjacent laths if they 
have $\mathrm{K}-\mathrm{S}$ and $\mathrm{N}-\mathrm{W}$ relations with parent austenite phase. As a result, the cleavage propagation will be stopped and diverged at the lath boundaries.

Fig. 3-13 Dilatometric measurement of AerMet 100 samples after (a) Normalization (N) treatment $\left(1150^{\circ} \mathrm{C}\right.$ for $\left.18 \mathrm{hrs}\right)$ and (b) Normalization (N) and Intercritical Annealing (L) treatment $\left(1150^{\circ} \mathrm{C}\right.$ for $18 \mathrm{hrs}$ and $677^{\circ} \mathrm{C}$ for 1 hr). Samples are heated to $900^{\circ} \mathrm{C}$ and then cooled down.

Fig. 3-14 TEM Bright field, dark field microstructure, and diffraction pattern for 9Ni Steel in the NQ condition (1423K, 18hrs for normalization; 1093K $1 \mathrm{hr}$ oil quenched; $77 \mathrm{~K} 1 \mathrm{hr}$ for chilling), showing that good crystallographic alignment remains in martensite packets.

Fig. 3-15 TEM micrographs of 9Ni steel in the NLQT condition (1423K, 18hrs for normalization; 963K, $1 \mathrm{hr}$ for intercritical annealing; 1093K, $1 \mathrm{hr}$, oil quenched; $77 \mathrm{~K}, 1 \mathrm{hr}$ for chilling). Local diffraction pattern indicates two variants within the same martensite packet.

Fig. 3-16 Fractography of J specimens of AerMet 100 with different heat treatments tested at $77 \mathrm{~K}$, indicating a decrease of cleavage size from NT, NQT to NLQT and NLQLQT. 
Fig. 3-17 Fractography of J specimen of AerMet 100 with different heat treatment tested at $77 \mathrm{~K}$, indicating a decrease of cleavage size from about $20 \mu \mathrm{m}$ in QT to $6 \mu \mathrm{m}$ in LQT and $3 \mu \mathrm{m}$ in LQLQT condition.

Fig. 3-18 J Testing results for AerMet 100 at 77K (a) J integral vs. da and (b) Load vs. strain curve, showing that grain refinement can stabilize crack propagation.

Fig. 4-1 Bain correspondence in the martensitic transformation of FCC to BCC or $\mathrm{BCT}$

Fig. 4-2 Illustrations of shear mechanisms (a) outside and (b) within close-packed planes in the $\gamma \rightarrow \alpha^{\prime}$ transformation proposed by Kudjumov and Sachs and Nishiyama.

Fig. 4-3 Illustration of (a) Bain Distortion has no invariant plane and (b) a second shear can generate a lattice invariant shear.

Fig. 4-4 Illustration of crystallographic relationship between initial austenite (FCC) and final martensite (BCC) in an $\mathrm{N}-\mathrm{W}$ related martensitic transformation.

Fig. 4-5 Illustration of crystallographic relationship between initial austenite (FCC) and final martensite (BCC) in a K-S related martensitic transformation. 
Fig. 4-6 Stereographic projection of $\{100\}$ pole figures for K-S and N-W related martensite in an austenite basis. Open and close marks represent K-S and $\mathrm{N}-\mathrm{W}$ relationships respectively. Circles, squares, and triangles represent the relations from Bain variant group $\mathrm{A}, \mathrm{B}$, and $\mathrm{C}$, respectively.

Fig. 4-7 Schematic illustration of formation of the large angle misorientation when the adjacent structure domains undergoes transformation with different Bain variants.

Fig. 4-8 An illustration of four-color theorem using US map

Fig. 4-9 Illustration of the effective grain size is (a) the packet size when constrained by prior austenite grain boundaries, (b) the block size when constrained by separately nucleated lath and (c) the individual lath when constrained by untransformed stable matrix.

Fig. 4-10 Stereographic projection of $\{110\}$ pole figures for K-S and N-W related martensite in an austenite basis. All solid labels represent N-W relationships while open ones represent K-S relations. The squares, circles, and triangles represent the relationships belonging to Bain variant group A, B and C, respectively. 
Fig. A-1 TEM micrographs of a 9Ni steel under a mechanical loading: (a) bright field, (b) dark field of mechanically transformed martensite and (c) dark field of the retained austenite, which still remains.

Fig. A-2 Schematic illustrations of retained austenite ahead of crack propagating along [001] cleavage plane of its nearby martensite.

Fig. A-3 Elastic energy of transformation under external stress field vs. $\theta$ angle, showing that at every location the retained austenite will transform to NW related martensite rather than continuing the same K-S variant as its adjacent lath. 


\section{List of Tables}

Table 1-1 Composition of typical high and ultrahigh strength steels

Table 3-1 Results of Charpy and J tests of AerMet 100 at $77 \mathrm{~K}$ for different heat treatments

Table 3-2 Results of uniaxial tensile and J tests of AerMet 100 with different heat treatments

Table 4-1 Re-grouping 24 K-S orientation relations into three Bain variant groups.

Table 4-2 Re-grouping $12 \mathrm{~N}-\mathrm{W}$ orientation relations into three Bain variant groups. 


\section{Chapter I. General Introduction}

The mechanical consideration that most often governs the initial selection of a structural alloy for engineering application is the combination of its strength (the ability to resist failure by plastic deformation) and toughness (the resistance to failure by crack propagation). However, strength and toughness typically have an inverse relation to each other; an increase in strength at a given temperature will generally leads to less plastic deformation, which relaxes the stress concentration around internal defects, and thus almost inevitably decreases the fracture toughness. This trade-off between strength and toughness is a recurring theme in the metallurgy of metals and alloys, especially in the field of high and ultrahigh strength steels. ${ }^{[1,2,3]}$

Recent studies in the field of steel research have been focused on how to push the limit of strength and toughness of high strength and ultrahigh strength steel. The steels that are of our interest here include high strength alloys, such as HY130, HY180, 6Ni and 9Ni steel, ultrahigh strength low-alloy steel, such as AISI 4340 and $300 \mathrm{M}$, secondary hardening steels, such as AF 1410 and AerMet 100, and maraging steels such as $18 \mathrm{Ni} 250-350$

grades. ${ }^{[4,5,6]}$ Typical compositions of these steels are summarized in Table 1-1 and are taken from various sources. Common to all of these steels is: 1) they contain the alloying element $\mathrm{Ni}$ with concentrations ranging from $1 \%$ to $18 \%$; and 2 ) they can be processed to have a lath martensitic structure and are usually used in this condition. The combinations of toughness and yield strength of these steels are shown in Fig. 1-1. 
Table 1-1 Compositions of typical high and ultrahigh strength steels

\begin{tabular}{|c|c|c|c|c|c|c|c|c|}
\hline Steel & $\mathrm{C}$ & $\mathrm{Ni}$ & $\mathrm{Mn}$ & $\mathrm{Co}$ & $\mathrm{Cr}$ & $\mathrm{Mo}$ & $\mathrm{V}$ & $\mathrm{Si}$ \\
\hline $5.5 \mathrm{Ni}$ Steel & 0.06 & 5.86 & 1.21 & -- & 0.69 & 0.20 & -- & 0.20 \\
\hline HY 130 & 0.08 & 5.11 & 0.84 & -- & 0.59 & 0.59 & 0.08 & 0.03 \\
\hline HY 180 & 0.1 & 10 & 0.15 & 8 & 2 & 1 & -- & -- \\
\hline 250 Maraging & 0.03 & 18 & -- & 8 & -- & 4.8 & -- & -- \\
\hline $300 \mathrm{M}$ & 0.42 & 1.8 & 0.7 & -- & 0.85 & 0.4 & 0.1 & 1.6 \\
\hline 4340 & 0.4 & 1.8 & 0.7 & -- & 0.85 & 0.25 & -- & 0.2 \\
\hline AF1410 & 0.16 & 10 & -- & 14 & 2 & 1 & -- & -- \\
\hline AerMet 100 & 0.22 & 10.9 & 0.05 & 13.7 & 3.0 & 1.1 & -- & 0.01 \\
\hline "high Cr" & 0.21 & 1 & 0.01 & 15 & 12.6 & 2 & -- & 0.5 \\
\hline
\end{tabular}

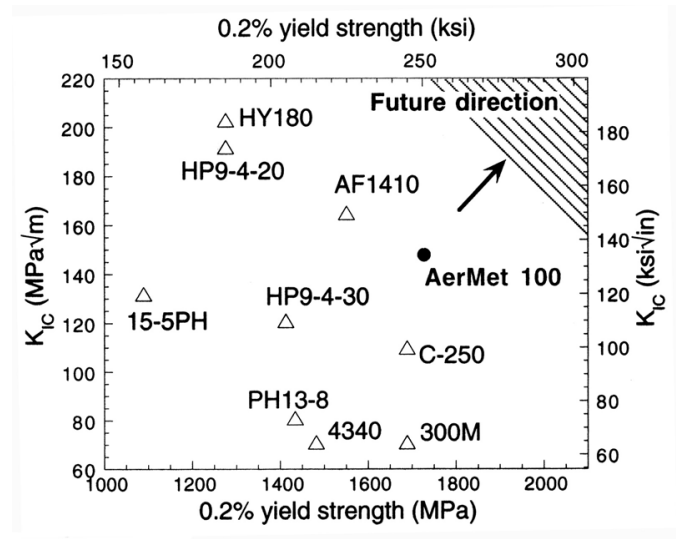

Fig. 1-1 Mechanical properties of typical high and ultrahigh strength steels 


\subsection{Fracture mode of steels}

In general, materials fail in one of three ways: 1) ductile fracture at high temperature or low stress, 2) transgranular cleavage and 3) intergranular separation at low temperature or high stress. The toughness of a high strength steel is determined, at least to first order approximation, by the fracture mode of failure. ${ }^{[7,8]}$

At room temperature, ferritic steels ordinarily fail in a ductile mode as a result of the nucleation, growth and coalescence of microscopic voids that initiate at inclusions and second phase particles (Fig. 1-2 (a)). The materials at the tip of the microvoids or other internal fatal flaws must yield plastically to relieve the applied stress before the normal stress reaches the value that is required to drive brittle fracture. As temperature decreases or as the applied stress or strain rate increases, the tendency towards brittle fracture may appear in one of the two modes: transgranular fracture along a certain crystallographic cleavage planes or intergranular separation along grain boundaries, whichever is easier. These modes are schematically shown in Fig. 1-2 (b) and (c), respectively.

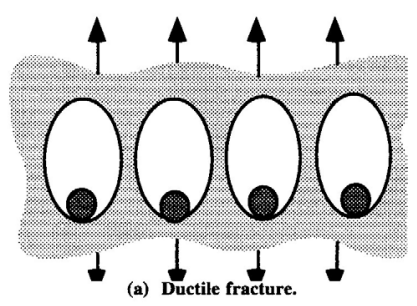

(a) Ductile fracture

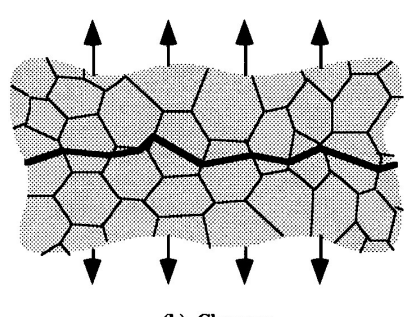

(b) Cleavage

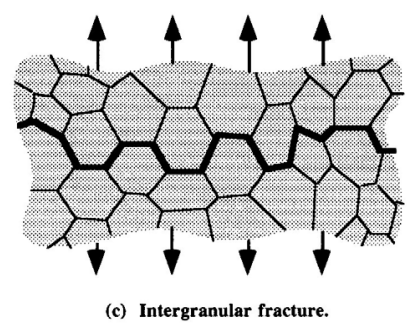

(c) Intergranular separation

(b) Transgranular cleavage

Fig. 1-2 Three typical failure modes of high and ultrahigh strength steels ${ }^{[7]}$ 
In high strength lath martensitic steels, the intergranular path is along the prior austenite grain boundaries. With the single exception of some high-manganese steels, intergranular fracture occurs due to the accumulation of embrittling species or precipitates along the grain boundaries. This behavior can be overcome by a combination of optimal melting practices used to minimize deleterious species, delicate composition control to "getter" residual impurities into relatively innocuous precipitates, and use of careful heat treatments to minimize the segregation at grain boundaries. Even in the case of high-Mn steels, where the weakness of grain boundaries seems to be an intrinsic property, intergranular fracture can be suppressed by adding a bit of Boron as a "grain boundary glue". ${ }^{[9,10]}$ As a consequence, the maximum attainable toughness of a high strength steel at low temperature is determined by its resistance to transgranular cleavage.

Since high strength steels fail in a ductile mode at high temperature and in a brittle transgranular cleavage mode at low temperature, they undergo through a ductile - brittle transition with decreasing temperature as shown in Fig. 1-3 (a). The qualitative source of the ductile - brittle transition is the competition between the yield strength, $\sigma_{\mathrm{y}}$, which controls the extent of plastic deformation, and the brittle fracture strength $\sigma_{\mathrm{F}}$, which controls the nucleation and propagation of the crack along its cleavage plane. This is illustrated in Fig. 1-3 (b). ${ }^{[11,12,13]}$ It is generally recognized that the yield strength will decrease as temperature increases whereas the fracture strength will remain almost unchanged with temperature. Thus, at temperatures above the ductile - brittle transition temperature (DBTT or $\mathrm{T}_{\mathrm{B}}$ ), as the applied stress increases towards failure, the stress at the tip of a pre-existing crack reaches yield strength before the brittle fracture strength is 
reached. Hence, extensive plastic deformation at the crack tip will relax the local stress and promote the ductile fracture, resulting in a high toughness value. On the other hand, at temperatures lower than $T_{1}$, the stress level will reach fracture strength before any significant plastic deformation, leading to a brittle fracture mode and a very low toughness value.

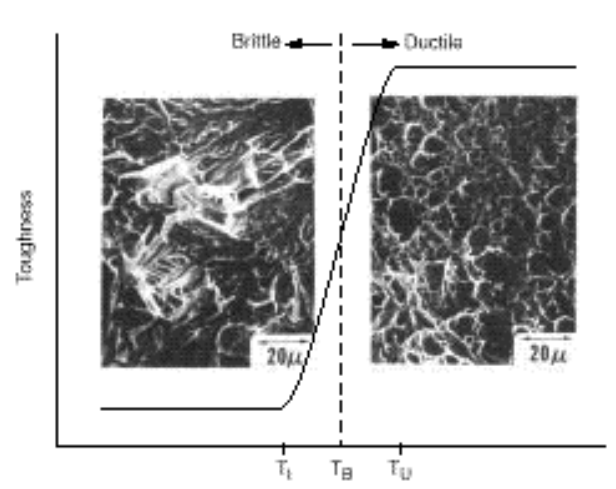

(a)

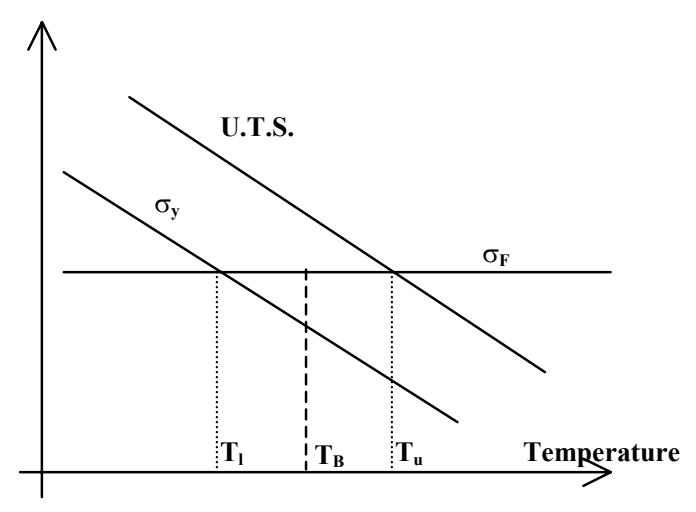

(b)

Fig. 1-3 (a) Typical ductile - brittle transition in high and ultrahigh strength steels ${ }^{[11]}$ and (b) a schematic drawing of Yoffee Diagram as an explanation of DBTT

It should be noted that the DBTT is strongly dependent on the testing methods used to evaluate it, due to variations in geometry, constraint, strain rate, etc.. For example, uniaxial tensile tests will produce a lower DBTT than notch bar tests, such as charpy and $\mathrm{K}_{\mathrm{IC}}$ tests. ${ }^{[14]}$ Ritchie et. al. also pointed out that even in the case of two notched specimens (e.g. a $\mathrm{J}_{\mathrm{IC}}$ and a Charpy, specimen), the DBTT might still be different due to different notch radii at the crack tip, and thus, different active volume involved during the fracture. ${ }^{[15]}$ Furthermore, even for the same type of $\mathrm{J}_{\mathrm{IC}}$ tests, the measured DBTT could also be different because of the effect of specimen thickness, and thus different constrain 
conditions. ${ }^{[16]}$ Therefore, DBTT is not an intrinsic material property and it is necessary to specify the testing method when the DBTT is stated.

In order for a high strength steel to have a high toughness value, the fracture must be ductile; the brittle transgranular crack propagation along cleavage plane must be made difficult. Therefore, the main topic of this dissertation is to increase the resistance to brittle cleavage fracture and thus ensure a ductile fracture mode for high strength steel. Once a ductile fracture mode is ensured, the achievement of high toughness involves the control of void nucleation, growth and coalescence, a subject beyond the scope of the present research. 


\subsection{Transgranular Cleavage Fracture}

Transgranular cleavage fracture can be defined as the rapid propagation of a crack along a particular crystallographic plane. ${ }^{[7]}$ The preferred cleavage planes are those with high packing density, low surface energy or lower nominal strength for separation. In the case of high strength ferritic steel with BCC structure, cleavage normally occurs on $\{100\}$ planes. ${ }^{[17]}$ The propagating crack changes direction everytime it crosses a grain boundary as it seeks the most favorable cleavage plane associated with the orientation of the grain and orientation of the external stress field ahead of the crack tip. It is important to include both macroscopic and microscopic consideration of cleavage fracture.

Macroscopically, cleavage fracture ordinarily happens when the local crack-opening stress exceeds the fracture strength $\sigma_{f}$ across a dimension greater than a characterized critical length $d \cdot{ }^{[18]}$ Fig. 1-3 (b) clearly illustrates the criterion that the stress exceeds the fracture strength. However, the consideration of a critical length is less obvious, but is just as necessary of a condition. It is well knows that the maximum stress in front of a macroscopic crack occurs ahead of the crack tip, and is notably a constant value in a small scale yielding approximation; the maximum stress is only a function of the yield strength of the materials, and is approximately 3 to 4 times the yield strength. However, the distance from the crack tip to the location where this maximum stress occurs increases with increasing materials toughness. ${ }^{[19]}$ It follows that if exceeding the critical fracture stress were a sufficient condition for cleavage fracture, any materials characterized by $\sigma_{\mathbf{f}}<3-4 \sigma_{\mathbf{y}}$ would fail upon the application of an infinitesimal load. 
Since this is not observed in practice, it is concluded that the stress criterion is a necessary but not a sufficient condition. From purely dimension consideration, the emergence of a length criterion is natural since the unit of fracture toughness involve both strength and length. This is observed in the stress intensity factor $\mathrm{K}_{\mathrm{IC}}\left(\mathrm{MPa}^{*} \sqrt{\mathrm{m}}\right)$ for the linear elastic case as well as in the $\mathrm{J}$ integral $\left(\mathrm{MPa}^{*} \mathrm{~m}\right)$ for elastic-plastic fracture mechanics. However the physical basis of the length criterion is established through microstructure considerations.

Microscopically, a cleavage fracture is rate-controlled by one of two events occurring in series: cleavage initiation around some local discontinuities in microstructure and transgranular cleavage propagation along preferred planes, whichever is more difficult. In both events, stress and length criteria are necessary.

It is generally recognized that cleavage will initiate around a local discontinuity, such as inclusions or second phase particles, ahead of a macroscopic crack when the local stress ahead of the crack tip exceeds the fracture strength. For a penny-shaped crack around a spherical particle, the fracture strength is

$$
\sigma_{f}=\left[\pi E G_{c f} / 2\left(1-v^{2}\right) d_{p}\right]^{1 / 2}
$$

where $d_{p}$ is the particle diameter. ${ }^{[20]}$ It is clear from Eqn. 1-1 that large particles corresponds to a low fracture strength such that crack initiation is easy. Thus, for a given remote stress, there exists a critical particle size beyond which cleavage is initiated. Since fracture occurs according to the weakest link mechanism, initiation of cleavage fracture will depend on the volume over which the stress is applied, since there exists a 
distribution of particle sizes. Therefore, both stress and length criteria are necessary for cleavage initiation.

After cleavage is nucleated at grain boundary particles or inclusions, it propagates along the most favorable $\{100\}$ cleavage plane in ferritic steels. Since crack propagation involves breaking bonds, the local stress must also be sufficient to exceed the bond strength. This is the stress criterion for cleavage propagation. However, the stress criterion is, again, necessary but not sufficient. Ritchie, Knott and Rice (RKR) stated that in order for a cleavage crack to propagate, the local tensile opening stress $\left(\sigma_{\mathrm{yy}}\right)$ directly ahead of the crack must exceed a local fracture strength $\left(\sigma_{f}\right)$ over a microstructurally significant characteristic distance. ${ }^{[18]}$ They found that the characteristic distance in ferrite/carbide microstructures is on the order of the spacing of the voids initiated around grain boundary carbides, typically about two grain diameters $\left(2 \mathrm{~d}_{\mathrm{g}}\right)$. They argued that if the fracture initiates around a grain boundary carbide, and propagates into a ferrite grain, then the stress must be sufficient to propagate the cleavage across the opposite grain boundary and into the next grain. Thus it is concluded that $\sigma_{\mathrm{yy}}$ must exceed $\sigma_{\mathrm{f}}$ over 1 to 2 grain diameters.

In summary, both stress and length criteria are essential to brittle transgranular cleavage fracture, for both nucleation and propagation processes. Therefore, in order to make the brittle fracture difficult in high strength steel, we have to take both fracture strength and characteristic length into account. In the following section, we discuss what affects brittle fracture, along with our approach to achieve high toughness in high strength steels. 


\subsection{General Considerations and Approaches}

Before we discuss the practical approaches to improve the toughness of high strength steels, it is necessary to consider and clarify the factors that affect brittle fracture at three different dimension scales: atomic, microstructural and macroscopic level.

As shown in Fig. 1-4 (a), it is well accepted that the fracture strength of transgranular cleavage is ultimately determined by events occurring on the atomic scale, since both initiation and propagation of a cleavage crack eventually involves breaking bonds. Although numerous models have been proposed to discuss the atomic aspects of brittle fracture, they all generally agree that the ideal fracture strength $\left(\sigma_{\mathrm{F}}\right)$ is controlled by the interaction between the electron potentials of neighboring atoms. ${ }^{[21,22]}$ Thus, both crystal lattice symmetry and alloying chemistry are very important to the ideal fracture strength. ${ }^{[23,24]}$ It should be noted that the ideal fracture strength $\left(\sigma_{\mathrm{F}}\right)$ discussed here is in general not equivalent to the local fracture strength $\left(\sigma_{f}\right)$ given by Equation 1-1. While the former is essentially the cohesive strength of a material as determined by the chemical bonds between neighboring atoms, the latter is not an intrinsic property of material, but is a microstructural parameter that depends upon the chemical composition and the processes history. The differences and relationship between the ideal and apparent fracture strength, and their effect on the DBTT will be discussed in detail in the Chapter II.

The characterized length criterion is fully determined on a microstructural level, as illustrated in Fig. 1-4 (b). For example, in the case of cleavage nucleation, the 
characteristic length is necessary because the system has to sample enough volume to find the particle with critical size around which the cleavage will be initiated. Hence, the characteristic length is determined by the distribution of geometric and size of second phase particles, such as grain boundary carbides, inclusions. For cleavage propagation, as proposed by RKR model, ${ }^{[18]}$ the characteristic length is about $1 \sim 2$ grain diameters, also a microstructural dimensions.

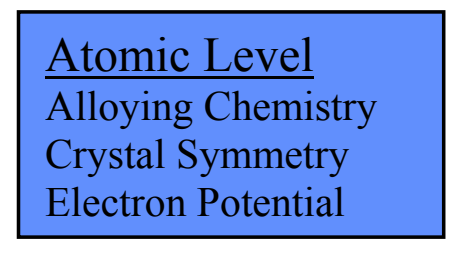

(a) Atomic Level -- Ideal Fracture Strength

\section{Microscopic Level}

Grain Structure

Dislocation Structure

Particles and Inclusions
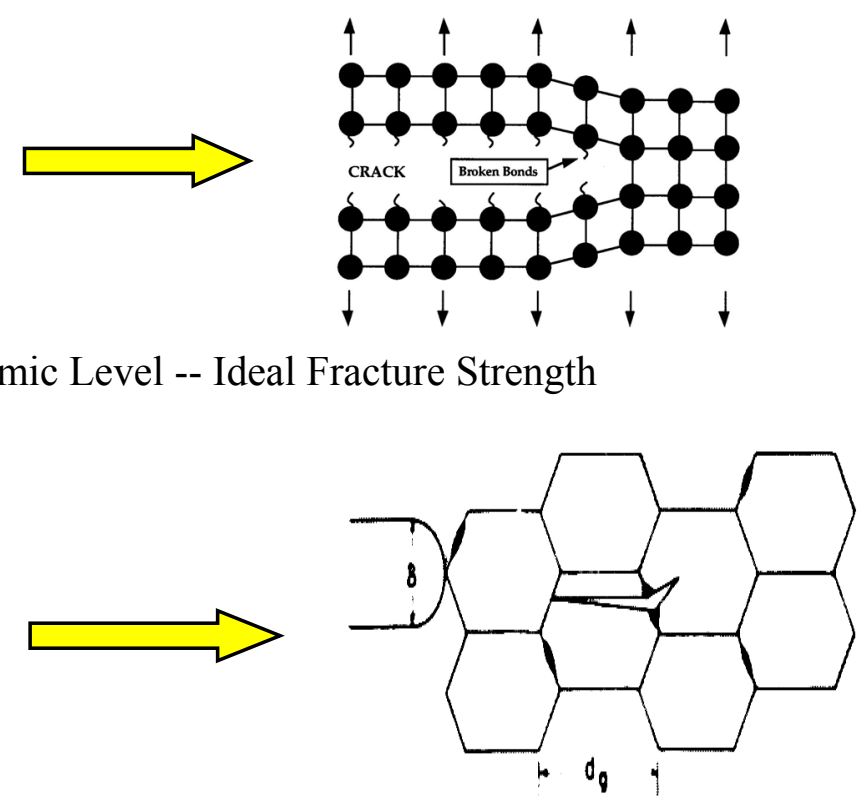

(b) Microscopic Level - Apparent Fracture Strength and Critical Length

\begin{tabular}{|l|}
\hline Macroscopic Level \\
\hline Sample Size and Geometry \\
Testing Methods \\
Crack Size and Tip Radius \\
\hline
\end{tabular}

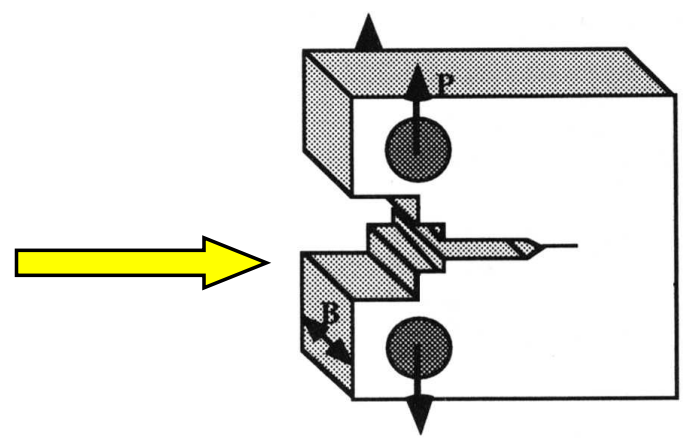

(c) Macroscopic level -- Stress Distribution ahead of the Crack Tip

Fig. 1-4 Schematic illustrations of factors in different levels that affect brittle transgranular fracture behavior of materials. 
It is noted that there are important interactions between atomic and microstructural scales. For example, dislocation structure, which bridges atomic and microscopic dimensions, can affect the fracture behavior of materials. Furthermore, the apparent fracture strength $\left(\sigma_{\mathrm{f}}\right)$ that can be measured experimentally is not fully determined at atomic scale, which controls the ideal fracture strength $\left(\sigma_{\mathrm{F}}\right)$, but it is also a function of microstructure, such as the size and distribution of precipitates, and the dislocation structure.

On a macroscopic scale, factors such as sample size and geometry, crack size and tip radius will affect the fracture behavior of a materials. However, they do not change the fracture criteria of materials: strength and critical length. Rather, such factors only change the stress distribution ahead of the crack tip. Thus, they will not affect materials intrinsic properties, such as $\mathrm{K}_{\mathrm{IC}}$, but just the ductile -- brittle transition temperature (DBTT). These factors are extrinsic and beyond the subject of this research.

Based on the considerations presented in this chapter, the two-fold approach taken in the study of high-strength and high-toughness steel presented in this dissertation will be focused on the two criteria for fracture:

1. Fracture Strength criterion: The ideal fracture strength will be discussed on atomic scale. The difference between ideal and apparent fracture strength will be addressed under different circumstances. The effect of alloying chemistry on ideal fracture strength will be investigated based on existing data. 
2. Characteristic length criterion: This is discussed on microstructural scale. Grain refinement will be studied, motivated by the increased difficulty to propagate a cleavage crack through grain boundaries.

As a preliminary example of these two approaches, we can consider the total work of fracture, expressed as $\mathrm{W}=\gamma^{*} \mathrm{~A}$, where $\gamma$ and $\mathrm{A}$ are surface energy and area of the fresh surface created during this fracture. The first approach is concerned with surface energy while the second approach is concerned with the surface area of the crack path. The goal in both approaches is to make brittle fracture more difficult, and therefore to increase the work for brittle fracture.

Chapter II will discuss the first approach on ideal and apparent fracture strength. Chapter III and IV will focus on the second approach, and will examine the thermal mechanism of grain refinement via martensitic phase transformation. Chapter III is based on experimental results of grain refinement while Chapter IV is focused on theoretical calculations based on martensitic transformation theory. 


\section{Chapter II. Fracture Strength Criterion}

In this Chapter, we will first discuss ideal and apparent fracture strength, and the significance of these properties in relations to the toughness and Ductile - Brittle transition temperature (DBTT) in high-strength steels. Then we shall examine and propose some practical approaches to push the limits of fracture strength in high strength steels.

\subsection{Ideal Fracture Strength}

The ideal fracture strength of a crystalline solid is limited by the elastic instability of its

crystal lattice under tension. ${ }^{[25]}$ The tensile stress associated with the elastic instability causes the crystal to break (if it has not already done so in response to pre-existing defects in the crystals), and defines the ideal fracture strength. Studies of the ideal fracture strength can be dated back to Frenkel $(1926)^{[26]}$ and Orowan (1949) ${ }^{[27]}$. Recent advances in computational techniques and computing machines have made it possible to make $a b$ initio calculations of the ideal fracture strength of crystals, via pseudopotential theory. In this section, we shall discuss those results and their consequences in details.

\subsubsection{Classical model for ideal fracture strength}

A material fractures in a brittle transgranular cleavage mode when sufficient stress and work are applied on an atomic level to break the bonds that hold atoms together. Fig. 2-1 shows schematic plots of the potential energy and force versus separation distance 
between atoms where the force curve is the first derivative of the energy curve. Equilibrium is defined as the spacing where the potential energy is at its minimum. In order to break bonds a tensile force that exceeds cohesive force is required.

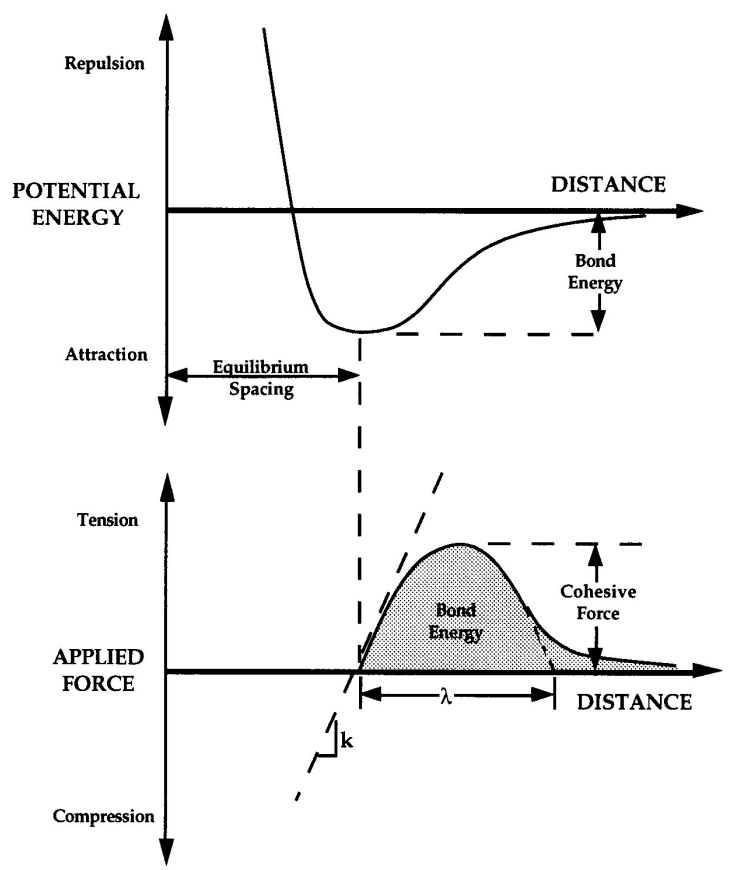

Fig. 2-1 Potential energy and force as a function of atomic separation ${ }^{[7]}$

Orowan proposed an approach to estimate the ideal fracture strength at the atomic level by idealizing the interatomic force-displacement relation as a half period of a sine wave, as shown in Fig. 2-1b. ${ }^{[27]}$ Assuming that the half-period length is $\lambda$, we have:

$$
P=P_{C} \operatorname{Sin}\left(\frac{\pi \times \Delta a}{\lambda}\right)
$$

where $\Delta \mathrm{a}$ is the displacement away from the equilibrium interatomic spacing, $\mathrm{a}_{0}$, and $\mathrm{P}$ and Pc are the applied and cohesive force, respectively. At the small strain limit, the displacement $\Delta \mathrm{a}$ is also small. Therefore, the equation can be simplified as: 


$$
P=P_{C} \operatorname{Sin}\left(\frac{\pi \times \Delta a}{\lambda}\right) \approx P_{C}\left(\frac{\pi \times \Delta a}{\lambda}\right)
$$

Since Hook's law is obeyed at the small strain limit, we find that

$$
E=\frac{\sigma}{\varepsilon}=\frac{P / A}{\Delta a / a_{0}}=\frac{P a_{0}}{\Delta a A}
$$

Substituting (2-2) into (2-3) and reorganizing terms, we will obtain:

$$
E=\frac{P a_{0}}{\Delta a A}=\frac{P_{c}}{A} \frac{a_{0} \pi \Delta a}{\Delta a \lambda}=\sigma_{C} \frac{a_{0} \pi}{\lambda} \Rightarrow \sigma_{C}=\frac{E}{\pi} \frac{\lambda}{a_{0}}
$$

Therefore the ideal fracture strength along a certain crystallographic direction is proportional to the elastic constant $E$ along the same direction, and $\lambda / \pi \mathrm{a}_{0}$ is the proportionality constant. If the half period length $\lambda$ is assumed to be equal to the atomic spacing $\mathrm{a}_{0}$, the ideal fracture strength is on the order of $0.3 \mathrm{E}$. This is the classic model of the ideal fracture strength.

\subsubsection{The strain at elastic instability}

Orowan's model is based on two important assumptions: 1 . The force verse displacement curve in the solid can be simplified as a sine wave; 2 . The elastic instability occurs at a strain of 0.5 . While the first approximation has been proven to be very reasonable, ${ }^{[28]}$ the second assumption requires further consideration.

Elastic instability occurs at the inflection point in the energy curve where the second derivative of energy is zero. This inflection point will be in between a stable phase and an unstable but stress-free phase. In the case of a simple crystalline structure, such as a 1-D or simple cubic structure, it is appropriate to assume that the unstable phase is generated 
when the atom has been pulled into the position of its nearest neighbor, leading to an elastic strain of $1(\lambda \approx a)$. As a result, the elastic instability occurs at the inflection point at a strain equal to 0.5 . However, in some other crystal lattices with high symmetry, things become more complicated.

In the case of a body center cubic (BCC) structure, for example, tension along $<100>$ directions, with a relaxed engineering strain of $26 \%$, will transform the structure to a face center cubic (FCC) structure, assuming constant volume (Fig. 2-2). In a non-magnetic material, both stable BCC and unstable FCC structures are unstressed by symmetry, and both are local minimum, so the tensile stress must pass at least one maximum along the transformation path.
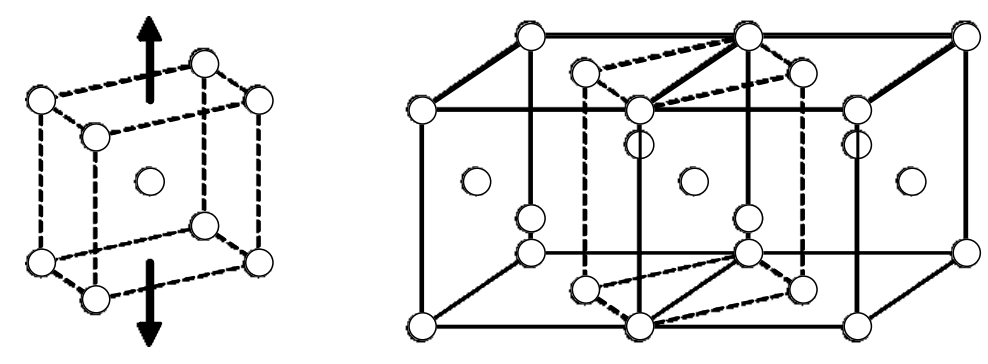

Fig. 2-2 The Bain strain connecting the BCC and FCC structures. If BCC is pulled in tension along [001] while contracting along [100] and [010], it generates an FCC crystal as shown.

This reverse Bain transformation sets a limit for the ideal fracture strength and $\lambda$ can be taken to be $0.26 \mathrm{a}_{0}$. Substituting this into Eqn. 2-4, we obtain

$$
\sigma_{C 100}=\frac{E_{100}}{\pi} \frac{\lambda}{a_{0}}=0.26 \frac{E_{100}}{\pi}=0.08 E_{100}
$$


This result is in a very good agreement with results from both ab intio calculations and experimental results. ${ }^{[29,30]}$ For example, the $a b$ intio result shows that the ideal fracture strength of $\mathrm{W}$ along $<100>$ direction is about $29.5 \mathrm{GPa},{ }^{[23]}$ which is $0.072 \mathrm{E}_{<100>}$. In addition, since no similar extrema are fixed by symmetry for the other possible direction of tensile loading, a BCC crystal has its minimum tensile strength along $<100>$ direction. This also explains why in general BCC crystals cleave on $\{100\}$ planes that are neither close-packed nor are the planes with the lowest surface energy.

However, when this method is applied to Fe or ferritic steels, the situation may be very different. Unlike the other BCC materials, such as Mo and W, the FCC phase in Fe is known to be only slightly higher than the BCC, and is at least meta-stable at low temperature. Therefore the engineering strain that brings the $\mathrm{BCC}$ to the FCC structure is equal to the whole period length instead of half of the period length. As a consequence, the ideal fracture strength should be only about $0.04 \mathrm{E}_{<100>}$, or equivalently $6 \mathrm{GPa}$ in the case of Fe. This number is too small to be credible since a much larger stress has been found ahead crack tips in ultra-high strength steels exhibiting elastic-plastic behavior and considerable ductility. ${ }^{[3]}$ Recent research has shown that a tensile strain along $<100>$ directions will bring the equilibrium $\mathrm{BCC}$ ferromagnetic phase of iron monotonically toward an unstable ferromagnetic FCC phase and the limit of stability will be reached before the alternate meta-stable FCC paramagnetic phase becomes favorable. Therefore, the point of elastic instability is governed not by the meta-stable paramagnetic FCC phase but by the unstable ferromagnetic FCC phase when $\lambda$ is about $0.28 \mathrm{a}_{0}$. Thus, the ideal fracture strength of iron and ferritic steel is $0.09 \sim 0.1 \mathrm{E}_{<100>}$, or equivalently $14.2 \mathrm{GPa}$. In 
conclusion, iron and other ferritic steel will cleave along $\{100\}$ planes and the ideal fracture strength is proportional to the elastic constant along this direction $\left(\mathrm{E}_{100}\right)$. 


\subsection{Apparent Fracture Strength and Ductile - Brittle Transitions}

Apparent fracture strength $\left(\sigma_{\mathrm{f}}\right)$, is a phenomenological strength value under which a micro-crack will be initiated and propagated from the weakest points in the sample. These weakest points may be $\{100\}$ cleavage planes in perfect single crystal of Fe, dislocation piled-ups at grain boundaries in the case of polycrystalline $\mathrm{Fe}$, or carbide precipitates or second phase inclusions in some ordinary steels. These factors will depend upon the chemical composition and microstructure of iron and steel. We shall discuss the meaning of apparent fracture strength and its effect on the ductile - brittle transition under each circumstance below.

\subsubsection{Flaw-free single crystal Fe}

In a flaw-free perfect single crystal $\mathrm{Fe}$, the apparent fracture strength $\left(\sigma_{\mathrm{f}}\right)$ is clearly equivalent to the ideal fracture strength $\left(\sigma_{\mathrm{F}}\right)$. In the previous section, we have discovered that the ideal fracture strength is proportional to the elastic constant along the cleavage direction. It has also been found that the ideal shear strength or yield strength in a perfect crystal is governed by symmetry and is proportional to the shear modulus along the easyglide direction. In the case of BCC iron and ferritic steel, the ideal shear strength is about $6.5 \mathrm{GPa}$ or $0.11 \mathrm{G}_{<111>{ }}{ }^{[23]}$

Therefore, the ductile - brittle transition in $\mathrm{Fe}$ is controlled by a competition between ideal fracture strength $\sigma_{\mathrm{F}}$ and ideal shear strength $\tau_{\mathrm{m}}$. For example, if we envision pulling a flawless crystal of $\mathrm{Fe}$ in tension along [100], the cleavage stress, 14.2GPa, would be 
reached when the resolved shear stress on [111](112) was 6.6GPa, which is approximately the ideal shear strength. Hence it is difficult to say whether this flawless crystal Fe would cleave in tension or deform in shear. In this case, defect-free $\mathrm{Fe}$ is inherently quasi-brittle. However, if the crystal is loaded in tension along other directions, such as $<111>$ or $<110>$, the crystal would first shear rather than cleave. It follows that the defect-free crystal of Fe can be ductile or brittle, depending upon the loading condition.

This argument only pertains to a flaw free crystal subject to uniaxial loading. The behavior of flaw-free materials subject to a stress concentration field associated with a sharp-tipped crack is even more complicated. The mechanical constraint on the stress field ahead of the crack tip has at least two effects, which counteract one another. First, the triaxial stress state increases the ratio of tension to shear and thereby promotes cleavage. However, the plain strain condition changes the strain path, which increases the ideal fracture strength and tends to suppress cleavage. In addition, if the shear stress at the tip of the crack is so high that dislocations are spontaneously emitted from the crack tip, the cleavage will also be suppressed. ${ }^{[31,32]}$

\subsubsection{Pure iron and plain steels with defects}

Realistically, materials always contain some defects, internal flaws, or dislocations that will affect the apparent fracture strength and thus the ductile - brittle transition of engineering materials, such as iron and ferritic steels. 
For instance, when dislocations are present, blunting of the crack tip before the critical load is reached for cleavage to initiate and propagate can complicate the situation. Dislocation motion is impeded by lattice friction, namely the Peierls - Nabarro stress, $\sigma_{\mathrm{p}}$, which is given by

$$
\sigma_{p}=\frac{2 G}{1-v} \exp \left(\frac{4 \pi h}{2(1-v) b}\right)
$$

where $\mathrm{G}$ is the shear modulus, $v$ is the Poisson's ratio, $\mathrm{h}$ is the interplanar spacing and $\mathrm{b}$ is the Burgers vector. ${ }^{[33]}$ For BCC Fe, the calculated value of the Peierls - Nabarro stress is about $0.01 \mathrm{G}(0.6 \mathrm{GPa})$. This value is far less than the ideal shear strength discussed in previous section. Even after we considered the other sources of resistance to dislocation motion, such as particles, inclusions, dislocation forests and grain boundaries, the presence of dislocations is still believed to greatly reduce the shear strength from ideal shear strength; and thereby always promotes a ductile fracture mode.

Another factor to be considered is micro-cracks that are usually initiated around some local discontinuities, such as dislocation piled-ups or grain boundary carbides and inclusions ahead of the macro-crack tip. Since a micro-crack sits inside the stress field ahead of the macro-crack tip, the remote crack opening stress applied to it is several times bigger than the externally applied stress due to the stress intensification factor of the macro-crack. Therefore, the maximum stress ahead of the micro-crack tip has been amplified twice leading to the ideal fracture strength. For example, if a penny-shaped crack was initiated around a particle in an intensified stress field ahead of the crack tip, the local stress, which is $3-4$ times the yield strength, can be considered as the remote stress applied to the micro-crack. Thus, the stress field ahead of this just-initiated micro- 
crack has been significantly amplified to more than ten times the external applied stress. Therefore, this microstructural discontinuity not only serves as a site for cleavage nucleation, but also amplifies the stress concentration due to the presence of the second crack. It follows that the apparent fracture strength in this case is generally several times lower than the ideal fracture strength of materials.

\subsubsection{High and ultrahigh strength steel}

In high and ultrahigh strength steel, additional considerations complicate the preceding analysis. In a high strength steel, typically characterized by a yield strength above $1 \mathrm{GPa}$, the crack opening stress $\sigma_{\mathrm{yy}}$ ahead of the macro-crack tip is so high that it already approaches the ideal fracture strength of materials. As a consequence, it may not be necessary to obtain the secondary intensification of stress field from micro-cracks. For example, in Aermat 100, when tested at $77 \mathrm{~K}$, the yield strength is about $2.2 \mathrm{GPa}$, whereas the nominal stress ahead of the crack tip approaches 10GPa according to elastic-plastic finite element analysis (FEM), as shown in Fig. 2-3. Thus, in the case of high and ultrahigh strength steel, the apparent fracture strength is about the same as ideal fracture strength. As a consequence, cleavage nucleation sites are not limited to local discontinuities sites, such as grain boundary particles, but may be anywhere in the lattice. Therefore, the ideal fracture strength is the relevant parameter to consider.

Moreover, it is well known that normal stress or crack opening stress $\sigma_{\text {yy }}$ ahead of a sharp crack can reach values that are more than four times the yield strength in an elasticplastic material. ${ }^{[19]}$ Since the ideal fracture strength for cleavage along $<100>$ is $14 \mathrm{GPa}$ 
in flaw-free $\mathrm{Fe}$, it follows that brittle transgranular cleavage should be the normal fracture mode in fracture toughness tests on steels with yield strength above 3GPa. This criterion sets up a limit for the combination of strength and toughness in iron and steels. This inference is at least qualitatively consistent with the known behavior of ultrahigh strength steels. It has been observed in our research that the samples of AerMet 100 at $77 \mathrm{~K}$ in a compact tension fracture toughness tests exhibit mixed ductile-cleavage mode and the yield strength of this ultrahigh strength steel is about $2.2 \mathrm{GPa}$ at $77 \mathrm{~K}$. However the tire codes example.

As a summary, the ideal fracture strength sets up a limit for the strength and toughness in steels and we are approaching this limit.

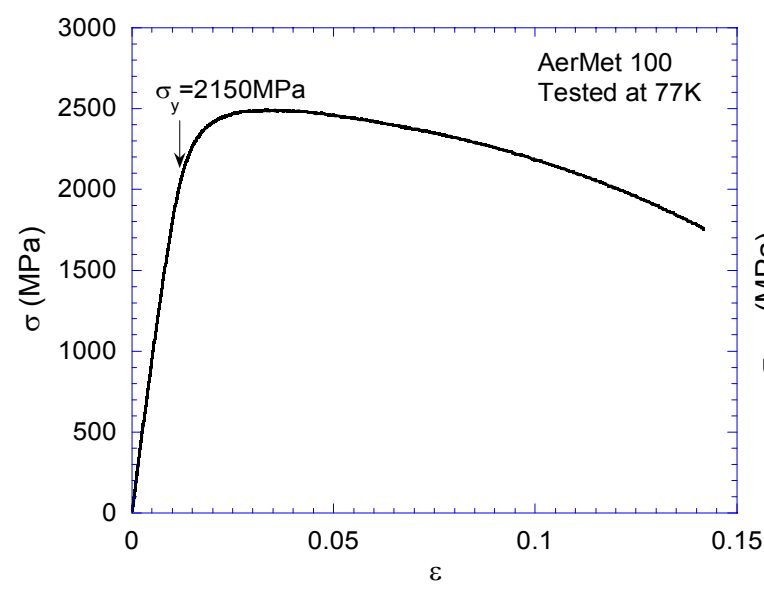

(a)

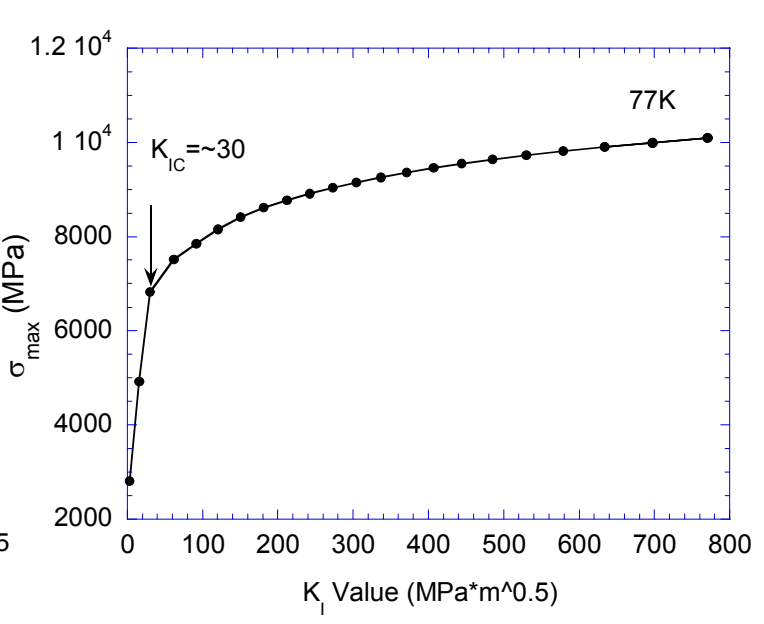

(b)

Fig. 2-3 (a) The stress-strain curve of AerMet 100 at 77K. (b) Elastic-plastic FEM solution for the maximum tensile stress as a function of the applied stress intensity. The value of $\mathrm{K}_{\mathrm{IC}}$ measured in a specific test is indicated. 


\subsection{Effect of Alloying Chemistry on Fracture Strength}

Since the properties of ultra-high strength steels are already approaching the limit of strength and toughness of $\mathrm{Fe}$, the question left to us is how to push this limit further. One possible area that can be exploited is alloying chemistry. Since both ideal fracture strength and shear strength along a certain crystallographic direction are closely related to its elastic constant, it is certainly worthwhile to examine the effects of alloying elements on the elastic constant and thus ideal strength.

It is well known that some alloying additions, such as $\mathrm{Mn}$, and $\mathrm{Ni}$, tend to lower the ductile - brittle transition temperature and increase the toughness of steels. The principle reason for this is microstructural. When used in high strength steel, these elements encourage the formation of dislocated martensite structures that combine good ductility with fine effective grain size. However, it has long been suspected that there is a chemical contribution as well, i.e. adding $\mathrm{Ni}$ and $\mathrm{Mn}$ can raise the intrinsic resistance to cleavage fracture. Unfortunately, to our knowledge there have been no ab initio studies of alloying effects on the ideal strength of $\mathrm{Fe}$, probably due to the difficulties in obtaining accurate local potentials of two-component alloys. This problem is made more difficult when an alloying element is magnetic, such as $\mathrm{Ni}$. Additionally, there is little experimental data available on the influence of $\mathrm{Ni}$ or $\mathrm{Mn}$ on the single crystal elastic constant of bcc iron, because it is technically very hard to make single crystals of BCC iron and its alloys. 
However, the polycrystalline elastic constants of bcc Fe-Ni alloys have been thoroughly studied. The results were compiled years ago by Ledbetter and Reed. ${ }^{[34]}$ The best fits to the polycrystalline elastic moduli give the relations:

$$
\begin{aligned}
& \mathrm{E}(\mathrm{GPa})=205-1.75 \mathrm{X}_{\mathrm{Ni}} \\
& \mathrm{G}(\mathrm{GPa})=81-1.12 \mathrm{X}_{\mathrm{Ni}}
\end{aligned}
$$

where $\mathrm{X}_{\mathrm{Ni}}$ is the Ni content in percentage by weight.

If we assume that $E_{100}$ and $G_{111}$ scale with $E$ and $G$, it follows that the ideal fracture strength $\left(0.08 \mathrm{E}_{100}\right)$ and ideal shear strength $\left(0.11 \mathrm{G}_{111}\right)$ will also scale with $\mathrm{E}$ and $\mathrm{G}$. As illustrated in Fig. 2-4, the ideal tensile strength decreases with increasing Ni content. The decrease is so substantial that the ideal cleavage strength of $\mathrm{Fe}-12 \mathrm{Ni}$ is more than $10 \%$ below that of pure Fe. However, the shear strength decreases even more rapidly: the ideal shear strength of $\mathrm{Fe}-12 \mathrm{Ni}$ is more than $16 \%$ below pure Fe. The dashed line in the figure shows the resolved shear stress on the most favorably oriented $<111>\{112\}$ slip system when the crystal is loaded to the cleavage stress along a $<100>$ direction. As $\mathrm{Ni}$ is added the resolved shear stress at the cleavage instability approaches the ideal shear strength, and the crystal becomes increasingly likely to shear before cleavage. In fact, this simple model predicts that a ferritic alloy with more than about $32 \% \mathrm{Ni}$ would be immune to cleavage. 


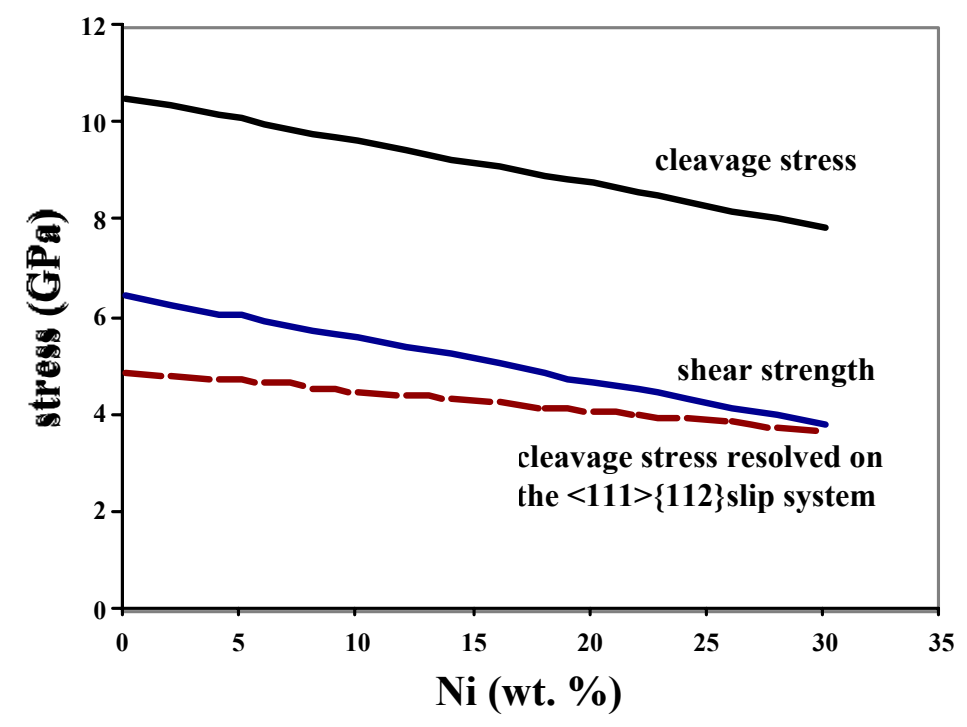

Fig. 2-4 The influence of $\mathrm{Ni}$ on the ideal cleavage and shear strengths of Fe. The lower dash line shows the resolved shear stress on $<111>\{112\}$ generated by the cleavage stress normal to $\{100\}$. 


\section{$2.4 \quad$ Future Work}

The discussions above give us a qualitative idea about how the addition of alloying element $\mathrm{Ni}$ can change the ideal fracture and shear strength and thus affect the ductile brittle transition of Fe based alloys. However, a further investigation on the effect of alloying element $\mathrm{Ni}$ is needed in order to fully understand the intrinsic effect of $\mathrm{Ni}$ on achieving good toughness in high and ultra-high strength steel and to find a chemical method to push the limit of strength and toughness of steels. This can be approached both theoretically and experimentally.

Theoretically the ideal fracture strength of Fe-Ni based alloys can be calculated ab intio, with some careful considerations. There are at least two major difficulties to accomplish this calculation. First, the electron potential of random solid solution itself is difficult to specify. Unlike the crystal lattices of pure elements, the exact type of atoms on a specific position in the crystal lattice is uncertain for random solid solution. It is essential to carefully model this uncertainty while maintaining the symmetry of the crystal lattice unchanged. Such calculations are underway within our group. ${ }^{[35]}$

Experimentally, it is of great interest to measure the elastic constant of Fe-Ni based alloy single crystals. However, there are several obstacles to conducting this work. First and foremost, it is very difficult to make bulk single crystals of the alloys of interest since most of them have a BCC structure. Rather than relying on bulk single crystals, it is possible to use large grains of material of interest in polycrystalline samples. This has 
been done for the measurement of the single crystal elastic constants of pure iron. However, the preliminary results showed that even at very high annealing temperature $\left(\sim 1200^{\circ} \mathrm{C}\right)$ and long annealing time ( $>24$ hours), the grains in Fe-Ni based alloys are less than several hundreds microns in diameter, much less than those achieved for the pure iron polycrystalline samples (several millimeters). The relatively small grain size is due to the presence of alloying element Ni. Nano-indentation has been used to investigate the elastic properties of materials within very small volumes. However, the complexity of the stress-state imposed make it very difficult to extract single crystalline properties from nano-indentation experiments.

There are some alternatives that have been successfully applied to ceramic materials, such as $\mathrm{Al}_{2} \mathrm{O}_{3}$ and $\mathrm{Si}_{3} \mathrm{~N}_{4}$ for which single-crystalline samples are also difficult to obtain. [36] The approach is based on a well-accepted assumption that the elastic constant of polycrystals is determined by the materials elastic constant of single crystals and its texture. With the innovation of computer technology, several software programs have been developed to compute the polycrystalline elastic moduli from knowledge of the texture and single crystal elastic constants of a sample. Therefore, it may be possible to infer the single-crystalline elastic constants from knowledge of the texture and elastic moduli of heavily deformed polycrystals.

For a series of Fe-Ni based alloys, such as pure $\mathrm{Fe}, \mathrm{Fe}-5 \mathrm{Ni}, \mathrm{Fe}-10 \mathrm{Ni}, \mathrm{Fe}-15 \mathrm{Ni}$ and $\mathrm{Fe}-$ $20 \mathrm{Ni}$, we shall first heavily deform the samples in order to form strong texture within them. Then their elastic constants and texture can be measured using standard ultrasonic 
wave techniques and X-ray diffraction method, respectively. After obtaining this information, we can substitute into the texture software, such as popLA from Los Alamos or BearTek from UC Berkeley to calculate the elastic constant of single crystal Fe-Ni alloys. The comparison of data from pure Fe with literature will be used to evaluate the accuracy of the method.

The approach introduced above will allow us to examine how the elastic constants, and thus ideal strength of single crystals $\mathrm{Fe}$ change with adding alloying element $\mathrm{Ni}$. Furthermore, since there is no reason to restrict this method to Ni only, it can also be applied to other alloying elements, such as Co, and $\mathrm{Mn}$, in order to investigate how the alloying chemistry will affect the ideal fracture strength and consequently the fracture behavior of steels. This may explain why some alloying elements will make steel tougher intrinsically, as well as provide us a guide on the direction of alloying techniques towards high-strength and high-toughness steel.

However, due to current limitations with equipment, this research has not yet been conducted. It is proposed that this work will be carried out within our group in the future. 


\section{Chapter III. Grain Refinement of Lath Martensitic Steel}

\subsection{Introduction}

\subsubsection{Why grain refinement}

Given the inverse relationship between strength and toughness, it is well known that grain refinement is only method that can contribute to both spontaneously. The yield strength of a material commonly obeys the relation known as the Hall-Petch equation: ${ }^{[37,38]}$

$$
\sigma_{y}=\sigma_{i}+k_{y} d^{-1 / 2}
$$

where $\sigma_{\mathrm{y}}$ is the yield strength, $\sigma_{\mathrm{i}}$ is the internal friction, $\mathrm{k}_{\mathrm{y}}$ is the Hall-Petch slope representing the resistance against the slip propagation across a grain boundary, and $\mathrm{d}$ is the grain size. The apparent fracture strength $\sigma_{\mathrm{f}}$ is derived from dislocation theory ${ }^{[39,40]}$ combined with classical Griffith theory ${ }^{[41]}$ for a brittle crack initiated around a dislocation pile-up at grain boundary with the result

$$
\sigma_{f}=4 G \gamma_{m} k_{y}^{-1} d^{-1 / 2}
$$

where $\sigma_{\mathrm{f}}$ is the apparent fracture strength, $\mathrm{G}$ is the shear modulus, $\gamma_{\mathrm{m}}$ is the surface energy, $\mathrm{k}_{\mathrm{y}}$ is the Hall-Petch slope and $\mathrm{d}$ is the grain size. Since the internal friction $\sigma_{\mathrm{i}}$ is a strong function of temperature, the yield strength $\sigma_{\mathrm{y}}$ of a ferric alloy increases significantly with decreasing temperature as shown in Fig. 1-3 (b). On the other hand, the apparent fracture strength $\sigma_{\mathrm{f}}$ only changes slightly with temperature, resulting in a typical ductile - brittle transition in ferritic alloys at temperature Tc where $\sigma_{\mathrm{y}}=\sigma_{\mathrm{f}}$ as following:

$$
T_{C}=C^{-1} \ln \left[B k_{y} d^{1 / 2} /\left(\beta G \gamma_{m}-k_{y}{ }^{2}\right)\right]
$$


where $\mathrm{C}, \mathrm{B}$ and $\beta$ are constants. ${ }^{[42]}$ Therefore, the transition temperature can be depressed by reducing the Hall-Petch slope $\mathrm{k}_{\mathrm{y}}$, increasing the surface energy $\gamma_{\mathrm{m}}$ and refining grain size. However, from Eqn. 3-1 and 3-2, it is apparent that grain refinement is the only means by which both yield strength and apparent fracture strength can be increased and the transition temperature can be simultaneously lowered.

\subsubsection{Approaches to grain refinement}

The general approach for grain refinement is thermomechanical processing, which is a combination of mechanical work to obtain large amount of plastic deformation and thermal exposure to induce recrystallization. This method has received dramatic emphasis in recent years with the launch of large "super steel" projects in Japan, Korea and China that are intended to develop the next generation of structural steels. ${ }^{[43,44]}$ It has been shown that exceptional strength and toughness combinations can be achieved in low alloy steels by refining grain size to the $1 \mu \mathrm{m}$ range.

However, there are still some barriers to implement these results successfully. It is very difficult to achieve the large amount of uniform plastic deformation that is necessary to produce ultrafine grain size through the thickness of plate steel. ${ }^{[45]}$ This is particularly true when the product of interest is a high strength, high alloy thick plate.

There are, however, alternatives to thermomechanical methods for grain refinement that are particularly applicable to high strength thick plates, bars and weldments. These are cyclic thermal treatments that exploit the properties of martensitic transformation and 
produce ultrafine grain size in lath martensitic steel. ${ }^{[46,47,48]}$ The practical impetus of grain refinement by thermal mechanism is the need for structure plates that have outstanding combinations of strength and toughness at low temperature. The scientific motivation is to push the limit of strength and toughness of high strength steels.

\subsubsection{Effective grain size in lath martensitic steels}

It is well known that ferritic steel cleaves along the $\{100\}$ planes. ${ }^{[3,7]}$ Therefore the effective grain size, in terms of transgranular cleavage fracture, can be defined as the coherent length on crystallographical cleavage planes, or the mean free path of crack propagation along $\{100\}$ planes in BCC ferritic steel.

In lath martensitic steels, however, the meaning of effective grain size is rather complicated and in fact a function of prior thermal processes. A typical microstructure of lath martensitic steel is shown in Fig. 3-1. ${ }^{[49]}$ The prior austenite grains are subdivided into packets of thin, closely aligned martensite laths. The structure within the packet appears to be very fine since the laths are less than a micron in cross-section. However, the diffraction pattern obtained from the interior of a packet (Fig. 3-1 d) revealed that the laths within a packet are in close crystallographic alignment and the lath boundaries are only low-angle boundaries. Hence, the packet is essentially a single crystal of martensite, and its size will define the effective grain size. This is illustrated in Fig. 3-2, which is a profile micrograph of cleavage fracture in an as-quenched $6 \mathrm{Ni}$ steel that was treated to produce very large packets and broken at $77 \mathrm{~K} .{ }^{[49]}$ The cleavage facets follow $\{100\}$ planes that are common to the laths in a packet, diverging at packet boundaries. 


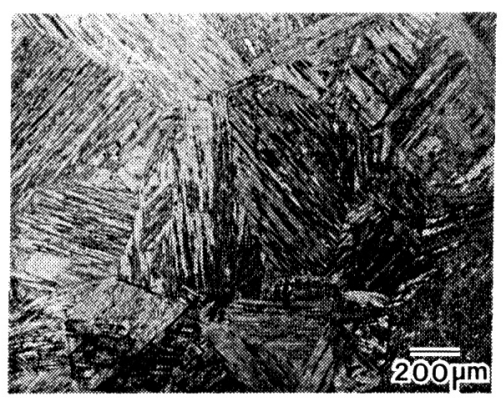

(a)

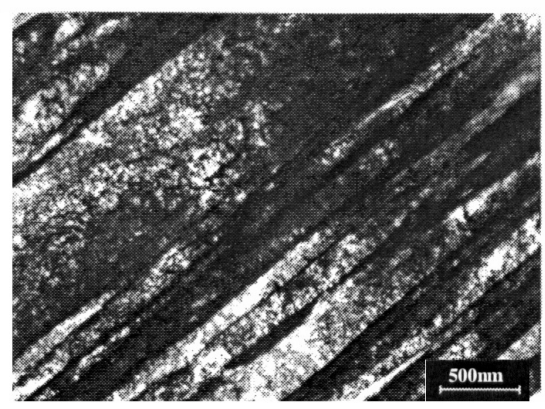

(c)

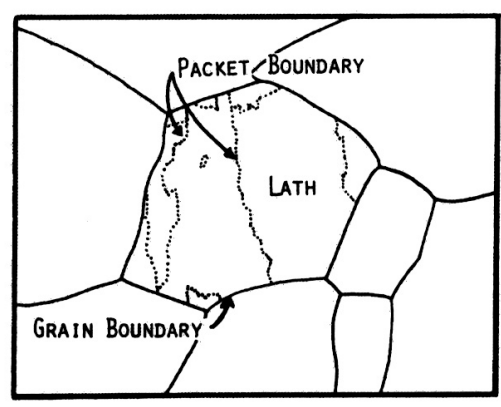

(b)

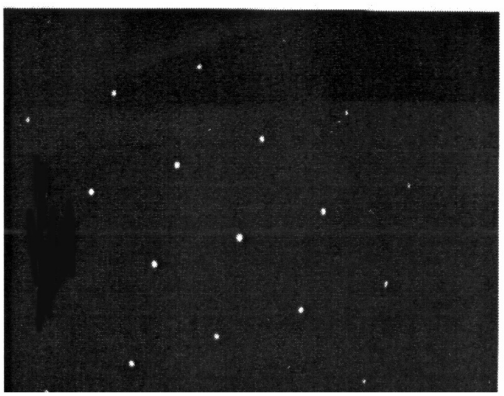

(d)

Fig. 3-1: The microstructure of a lath martensitic steel. (a) Optical microstructure of lath martensitic Steel; (b) Illustration of subdivided packet within a prior austenite grain; (c) dislocated lath martensite within a packet showing alignment of laths of less than $0.5 \mathrm{~mm}$ thickness; (d) The diffraction pattern shows the laths are almost identical in crystallographic orientation. (Courtesy of Y.H. Kim and T. K. Lee ${ }^{[49]}$ ) 


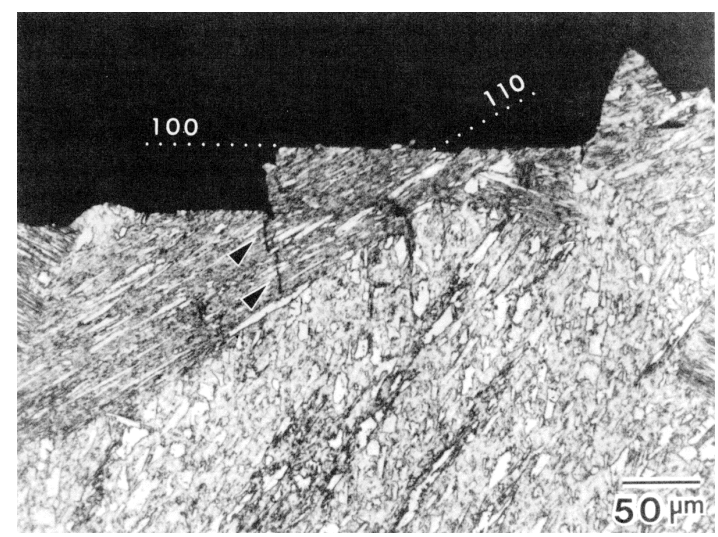

Fig. 3-2 Scanning electron micrograph of etched cross-section of lath martensitic steel broken below $\mathrm{T}_{\mathrm{B}}$. The cleavage cracks follow common $\{100\}$ planes across parallel laths in a packet, indicating that the effective grain size is the packet size. (Courtesy of Y. H. Kim ${ }^{[49]}$ ) 


\subsubsection{General approach of grain refinement in lath martensitic steel}

Given the nature of the lath martensite and the definition of effective grain size, there are three basic paths to grain refinement. The first approach is to refine the packet size by decreasing the prior austenite grain size. ${ }^{[48,50]}$ The thermal treatment to accomplish this is Austenite Reversion (Fig. 3-3 (b)), followed by a quenching to re-transform to martensite before the austenite can grow to appreciable size. This is referred to a "quench" or "Q" treatment, which is generally included in the processing of lath martensite steels. However, it has proved difficult to achieve prior austenite grain size below $10 \mu \mathrm{m}$ with Austenite reversion treatment only. ${ }^{[50]}$

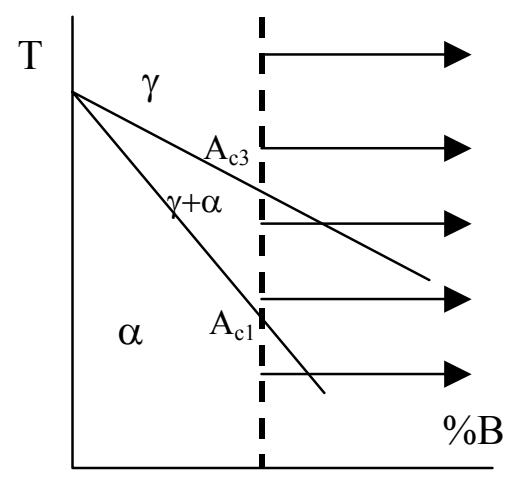

(a) Normalization $(\mathrm{N})$

(b) Austenite Reversion (Q)

(c) Intercritical Annealing (L)

(d) Intercritical Tempering $(\mathrm{T})$

(e) Tempering ( $\mathrm{t}$ )

Fig. 3-3 Schematic phase diagram of the five heat treatments used in this research.

The second method of grain refinement is to separate the lath using thin islands of thermally stable austenite. This is accomplished by tempering the alloy at a temperature just above $A_{C 1}$ (the boundary between the single-phase $\alpha$ region and the two-phase region) as shown in Fig. 3-3 (d)) to precipitate the small islands of alloy-rich austenite along the martensite lath boundaries. The mechanism by which this austenite can lead to an increase in toughness is still not clear. One of the possible explanations relies on the 
fact that the precipitated austenite is not mechanically stable, but transforms to fresh martensite when the alloy is deformed. ${ }^{[51,52]}$ However, the crystallographic variant (i.e. unique orientation relationship between martensite and parent phase austenite) adopted by this fresh martensite is determined by the applied stress rather than the crystallography of the packet. ${ }^{[53]}$ Therefore, the fresh martensite with a different variant from the dominant one of the packets will break up the alignment of the laths. This "intercritical tempering" (T) treatment is used in the traditional "QT" treatment of 9Ni steel ${ }^{[54,55,56]}$ and in the "QLT" treatments of 5-6Ni steels ${ }^{[57,58,59]}$ to toughen them for service at cryogenic temperatures. However, it is difficult to refine the whole package thoroughly due to the difficulties to obtain a dense distribution for austenite precipitation along every lath boundary.

The third method of grain refinement is to directly disrupt the crystallographic alignment of martensite laths within a packet and generate a large angle misorientation between the adjacent lath using thermal methods. ${ }^{[60,61]}$ Although this seems to be an ultimate way to completely refine the whole packet and create a material with submicron effective grain size, there is only little effort made using this method, possibly due to the difficulties to control the martensitic transformation and the resulting crystallographic relationship with the parent austenite phase. Thus, the research discussed here is intended to clarify the thermal mechanism which can directly break alignment within packets for grain refinement in lath martensitic steels and to understand their impacts on mechanical properties. 


\subsection{Experimental Procedures}

The material used in this research is AerMet 100 (Fe-13.4Co-11.1Ni-3.1Cr-1.2Mo0.23C) ${ }^{[62]}$ and $9 \mathrm{Ni}$ Steel. A secondary-hardening steel developed by Carpenter Technologies, AerMet 100 not only offers the exceptional combination of strength and toughness, but also appears to have submicron effective grain size after a standard QT treatment (1158K for $1 \mathrm{hr}$ for austenization and $765 \mathrm{~K}, 5 \mathrm{hrs}$ for tempering). TEM micrographs in Fig. 3-4 illustrate that there are two variants within a packet and the laths are decomposed into crystallographically distinct subvolumes that are smaller than $0.5 \mu \mathrm{m}$ in size. Our research is initiated based on this mystery since it is believed that standard QT will not break the crystallographic alignment within the packets.

In order to establish a homogeneous initial microstructure for grain refinement studies, All samples of AerMet 100 were first annealed at $1423 \mathrm{~K}$ for 18 hours for a normalization treatment $(\mathrm{N})$. (Fig. 3-3 (a)) The time and temperature were chosen to homogenize the solute species $(\mathrm{Ni}$ and $\mathrm{Co}$ ). Then a series of heat treatments were performed on these $\mathrm{N}$ treated alloys, including a Q treatment (austenite reversion at $1158 \mathrm{~K}$ for 1 hour), an L treatment (intercritical annealing at 950K for 1 hour), and an LQ treatment (intercritical annealing at $950 \mathrm{~K}$ for 1 hour, then austenite reversion at $1158 \mathrm{~K}$ for 1 hour). After heat treatment, all samples were oil quenched, and then chilled at $77 \mathrm{~K}$ for 1 hour to complete martensitic transformation and tempered at $755 \mathrm{~K}$ for 5 hours. 
Similar heat treatment circles were also applied on $9 \mathrm{Ni}$ steel in order to avoid the chemical effects of Co and other alloy elements on grain refinement of lath martensitic steel. For $9 \mathrm{Ni}$ steel, the normalization treatment (Q) is also $1423 \mathrm{~K}$ for $18 \mathrm{hrs}$. However, the intercritical annealing (L) treatment is $963 \mathrm{~K}$ for 1 hour and the austenite reversion (Q) treatment is $1093 \mathrm{~K}$ for 1 hour.

Microstructure has been examined after each heat treatment using both optical microscopy and transmission electron microscopy (TEM). Orientation relationship between adjacent lath has been investigated by selected area diffraction pattern and dark field images. Composition profiles between adjacent laths were examined using analytical electron microscopy (AEM) and EDAX. Additional dilatometer tests were also performed to measure the $\mathrm{M}_{\mathrm{S}}$ temperature of martensitic transformations.

Finally, uniaxial tensile, charpy and $\mathrm{J}$ tests were conducted at room temperature and $77 \mathrm{~K}$ for different heat treatment conditions to study the effect of grain refinement on cleavage propagation. In addition, a sample with a cyclic LQLQ heat treatment was tested to examine the effect of double treatments. Fracture surfaces were examined using scanning electron microscopy (SEM). 


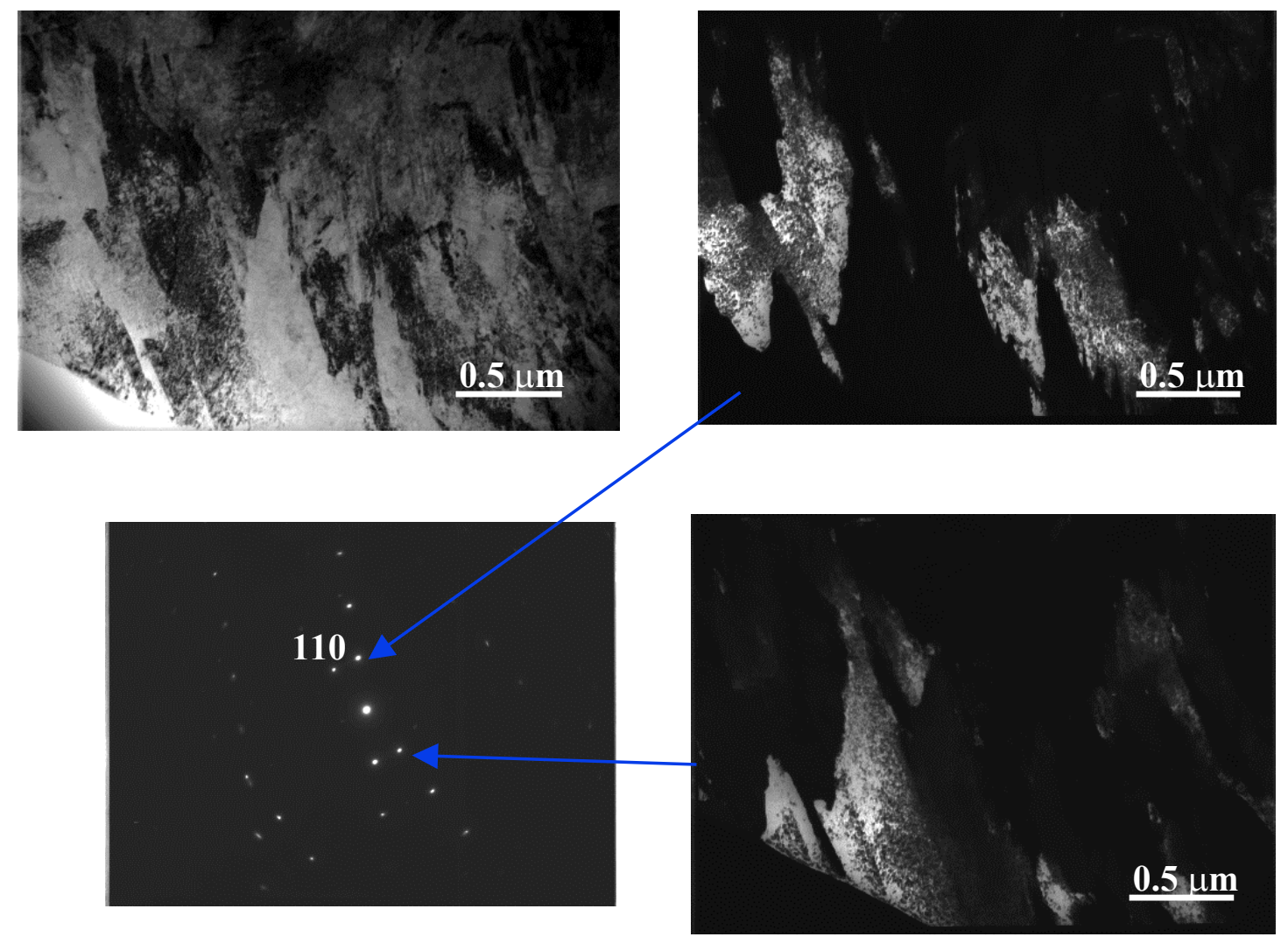

Fig. 3-4 TEM microstructure of AerMet 100 in a standard QT condition $(1158,1 \mathrm{hr}$, oil quenched; $77 \mathrm{~K} 1 \mathrm{hr}$ for chilling; $755 \mathrm{~K}$, 5hrs for tempering). Local diffraction pattern indicates two sets of diffraction pattern (variants) in a martensite packet. 


\subsection{Microstructural Evolutions}

\subsubsection{Normalization $(\mathrm{N})$ treatment}

Normalization treatment serves two purposes: to create a homogeneous and coarse initial structure and, more importantly, to investigate whether the fact that there are more than one variant within a martensite packet is an intrinsic property of alloys. Fig. 3-5 shows the microstructure of AerMet 100 obtained after an $\mathrm{N}$ treatment followed by quenching to room temperature and tempering. The prior austenite grain size is coarse, on the order of $150 \mu \mathrm{m}$. The TEM image shows dislocated martensite laths are well aligned and share the same variant within packets, indicating that the misalignment between adjacent laths is removable, therefore, not an intrinsic property of alloys but a result of heat treatment.

\subsubsection{Reversion (NQ) treatment}

After normalization, the alloy underwent a standard QT treatment as indicated by the manufacturer. This treatment was used to refine the prior austenite grain size and to determine the degree of lath alignment after reversion to just above $\mathrm{A}_{\mathrm{C} 3}$ (Shown in Fig. 33). Fig. 3-6 showed the microstructure of AerMet 100 in the condition of the "NQT". The prior austenite grain size has been significantly refined to about $50 \mu \mathrm{m}$. However, local area diffraction patterns taken from the interior of packets show a single-variant pattern, indicating that a standard QT treatment can only lead to a decrease in prior austenite grain size, but not a disruption in crystallographic alignment within the packets. 
The mechanism of reverse martensitic transformation and its effects on refinement of prior austenite grain size during reversion treatment $(\mathrm{Q})$ are still not entirely clear. ${ }^{[63,64]} \mathrm{It}$ is generally accepted that at low temperature where the diffusivity of solute species is low, the dominant mechanism of reverse austenization is diffusionless cooperative movement of atoms. ${ }^{[65]}$ One example is the reverse austenite transformation in stainless steel. ${ }^{[45]}$ On the other hand, when reverse austenization temperature $\left(A c_{1}\right)$ is high, such as in most lath martensitic steels, the transformation will proceed through nucleation and growth of austenite. ${ }^{[66]}$ Fig. 3-7 shows optical microstructure of AerMet 100 after heattreated at $1423 \mathrm{~K}, 18 \mathrm{hrs}$ for normalization; and $1158 \mathrm{~K}$ for 1 minute and 2 minutes. The change in microstructure indicates that new small austenite grains nucleate and grow from grain boundaries (in most cases) and inside grains (sometimes). This has been believed to be the reason that reversion treatment can refine prior austenite grain size. 

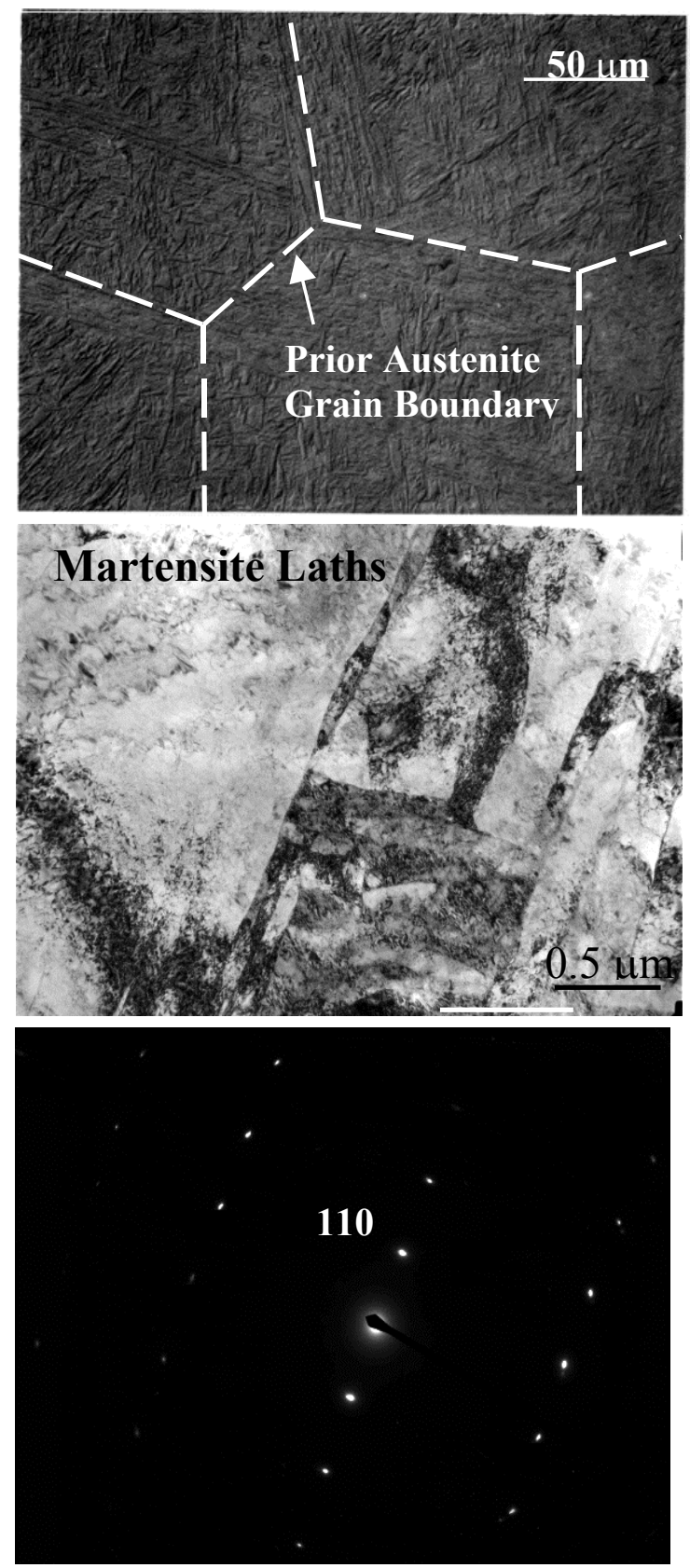

Fig. 3-5 Optical and TEM Microstructure of AerMet 100 after the N treatment (1423K, 18 hours for normalization; 77K, 1 hour for chilling), showing large prior austenite grains. Diffraction patterns taken inside the packets exhibit only one variant, indicating well aligned martensite laths 

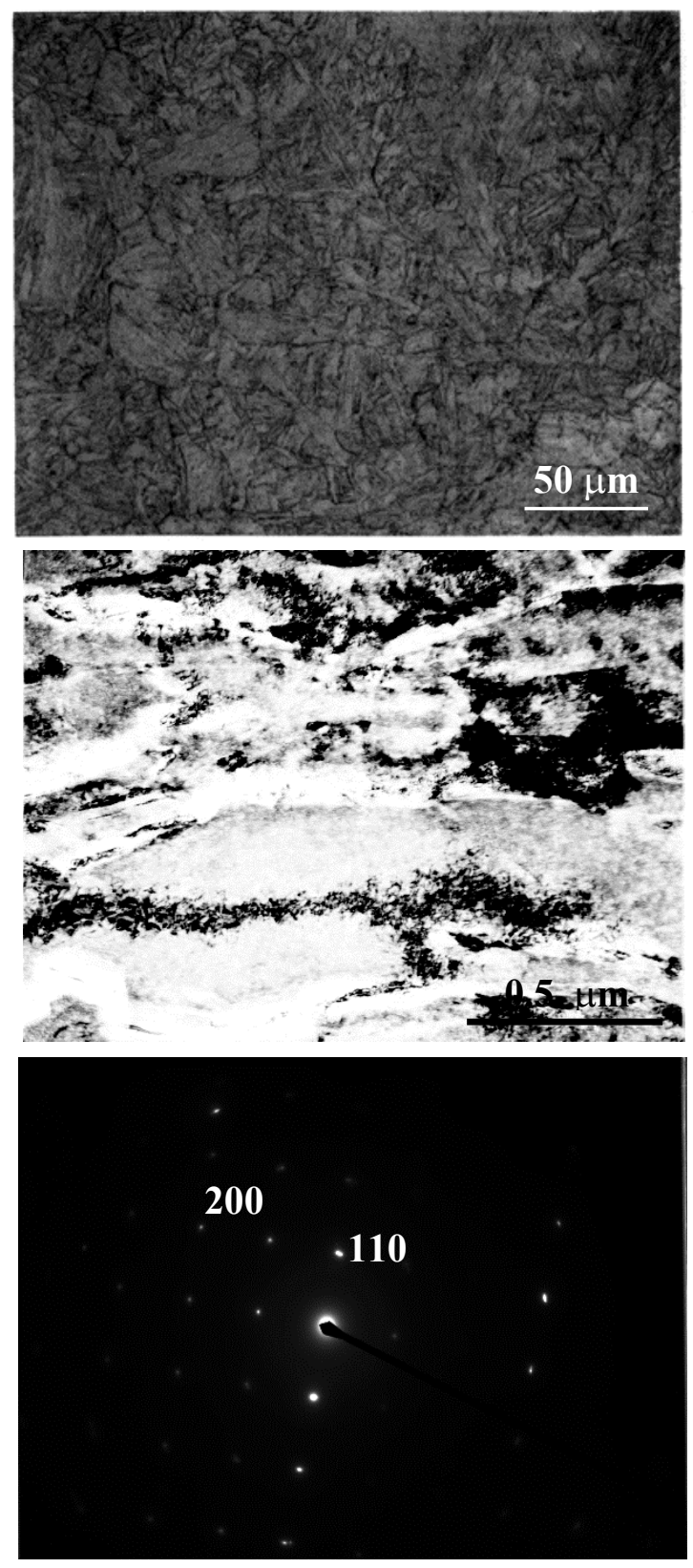

Fig. 3-6 Optical and TEM microstructure of AerMet 100 in the NQT condition (1423K, $18 \mathrm{hrs}$ for normalization; $1158 \mathrm{~K} 1 \mathrm{hr}$ oil quenched; $77 \mathrm{~K} 1 \mathrm{hr}$ for chilling; $755 \mathrm{~K}$, $5 \mathrm{hrs}$ for tempering), showing prior austenite grains have been significantly refined. However, the diffraction pattern taken within a packet still indicates that good crystallographic alignment remains in martensite packets. 


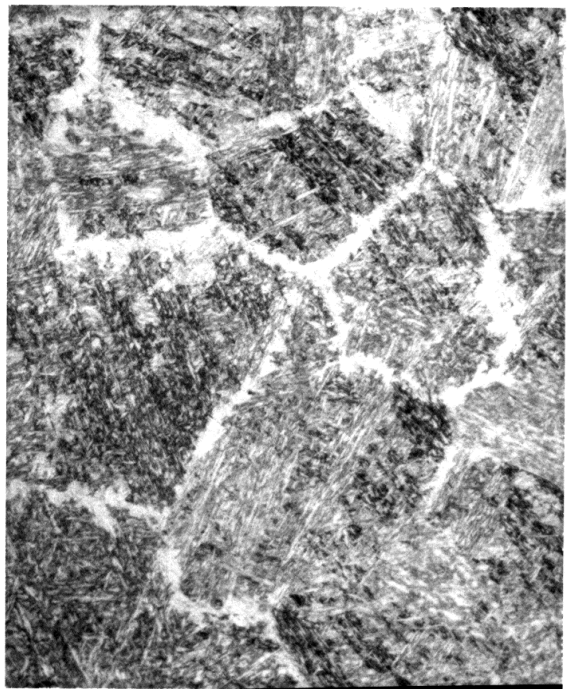

(a)

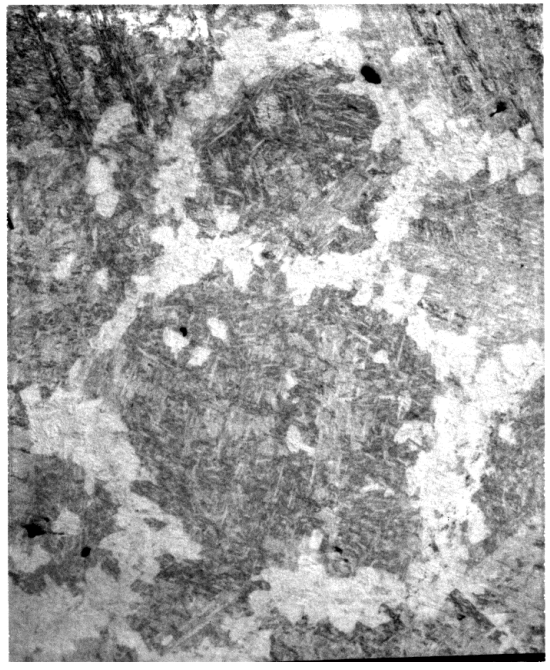

(b)

Fig. 3-7 Optical microstructure of AerMet 100 after heat-treated at 1423K, $18 \mathrm{hrs}$ for normalization; and $1158 \mathrm{~K}$ for (a) 1 minute and (b) 2 minutes, indicating that recrystallization occurs through nucleation and growth of new austenite grains from grain boundaries in most cases and sometimes inside grains. 


\subsubsection{Intercritical annealing (NL) treatment}

An intercritical annealing is accomplished by holding the alloy in a temperature near $\mathrm{A}_{\mathrm{C} 3}$ (in this case, $950 \mathrm{~K}$ for $1 \mathrm{hr}$ ), and in the top of the two-phase $(\alpha+\gamma)$ region as shown in Fig. 3-3 (c). After quenching to room temperature, the resulting microstructure (Fig. 3-8) is a lamellar mixture alternating between laths of highly-dislocated fresh martensite and those of well-recovered original martensite, with the same variant inside a packet. However, L treatment had little effect on the prior austenite size, which is about the same as $\mathrm{N}$ treatment. EDAX results across the laths in Fig. 3-9 shows the composition difference in eight adjacent laths: the fresh martensite $\left(\alpha_{\mathrm{f}}^{\prime}\right)$ is rich in alloy content Ni but relatively lean in Co, while the recovered martensite $\left(\alpha_{t}^{\prime}\right)$ is lean in Ni but rich in Co, indicating that the alloy has become a "dual phase" lamellar steel. At the same time, the original martensite recovers, losing most of its dislocation density.

According to phase diagram (Fig. 3-3), it is obvious that the alloy can develop this dualphase $(\alpha+\gamma)$ structure during intercritical annealing, primarily through the nucleation and growth of austenite along the martensite lath boundaries as shown in Fig. 3-10. Austenite generally will first nucleate at the lath boundary where $\mathrm{C}$ concentration is high. The following growth of precipitated austenite is accompanied by solute Nickel diffused into austenite and Cobalt diffused away from austenite since $\mathrm{Ni}$ is an austenite stabilizer while Co favors ferrite more than austenite. ${ }^{[67]}$ In addition, the interdiffusion between Co and Ni can also help stabilize further the lattices of austenite and martensite. The final morphology of precipitated austenite during intercritical annealing can either be island or

thin film shape, depending upon the thermodynamics and kinetics during the growth. ${ }^{[68]}$ 
In our research, precipitated austenite will have a highly-coherent interface with surrounding martensite. Thus, small interface energy between austenite and martensite will thermodynamically favor a growth along lath boundaries to create a thin layer of precipitated austenite. On the other hand, the interdiffusion between $\mathrm{Co}$ and $\mathrm{Ni}$ also mainly occurs along lath boundaries since they are the fast diffusion paths. Hence, the thin film morphology is favored by both thermodynamics and kinetics.

The alloy-rich austenite re-transforms to martensite almost completely on the subsequent cooling to room temperature and following chilling at $77 \mathrm{~K}$. Probably because its transformation is catalyzed by the martensite that surrounds it, this austenite has a strong tendency to transform back into the specific martensite variant of the surrounding packet to minimize the surface energy. Although this treatment does not refine grain size directly, it prepares the microstructure for effective grain refinement in subsequent steps. 

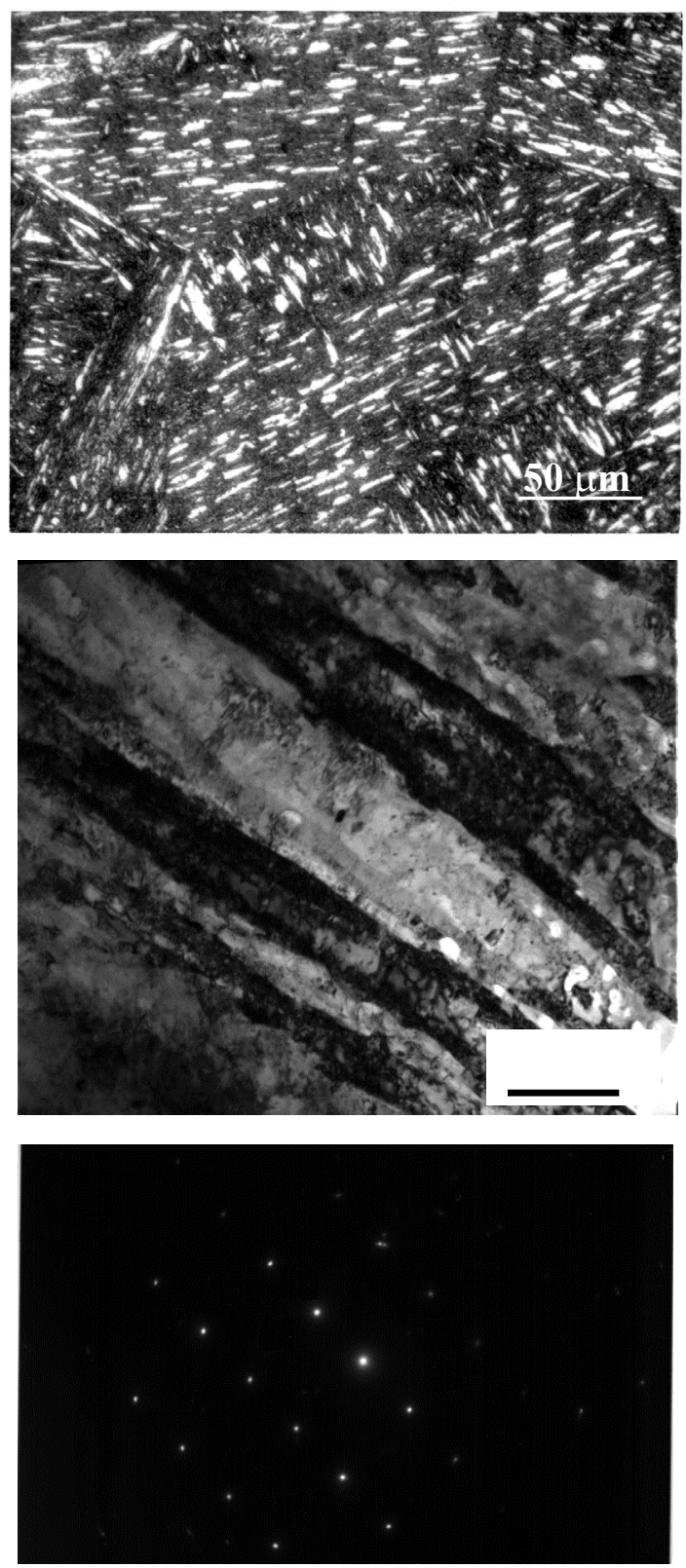

Fig. 3-8 Optical and TEM microstructure of AerMet 100 in NL condition (1423K $18 \mathrm{hrs}$ for normalization; 950K, $1 \mathrm{hr}$ for intercritical annealing), showing a lamellar pattern alternating between heavily-dislocated fresh martensite laths and tempered martensite laths. However, diffraction pattern exhibits only one variant within the packet. 

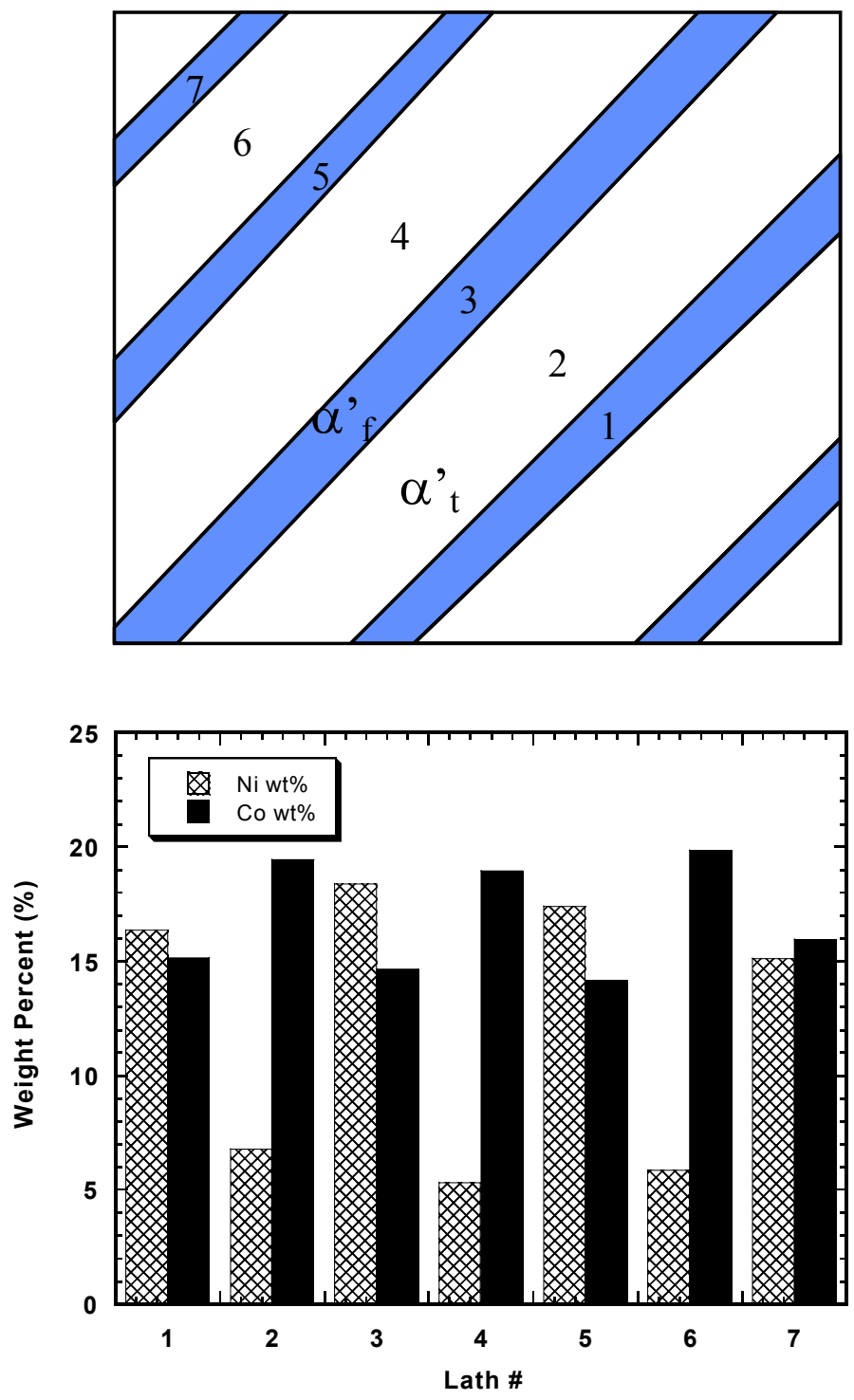

Fig. 3-9 EDAX results on individual lath of AerMet 100 in NL condition (1423K 18hrs for normalization; 950K, $1 \mathrm{hr}$ for intercritical annealing) revealed that solute distribute alternatively in the adjacent laths in a lamellar pattern. However, Ni and Co have opposite trends since $\mathrm{Ni}$ is austenite stabilizer and Co favors ferrite. 


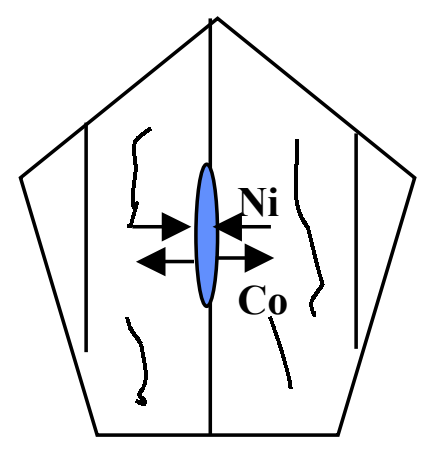

(a)

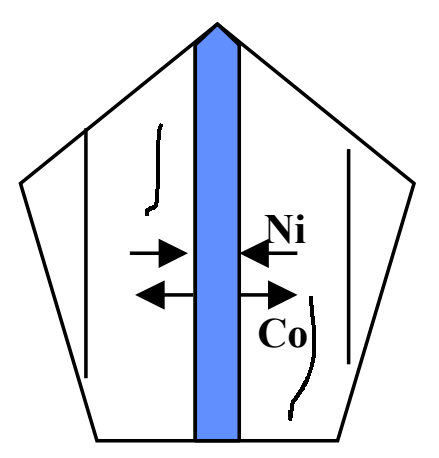

(b)

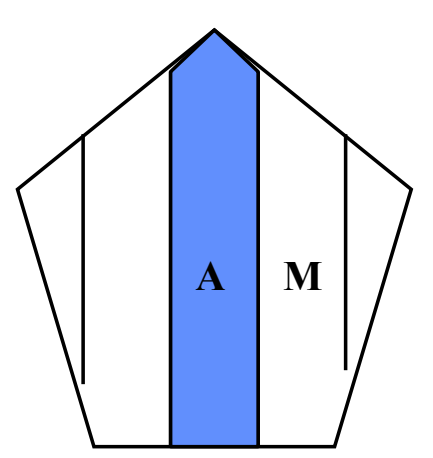

(c)

Fig. 3-10 Schematic Illustrations of (a) austenite nucleation at lath boundaries, (b) austenite growth along lath boundary to form a thin layer of precipitated austenite and (c) adjacent martensite laths have been tempered during intercritical annealing treatment (L), resulting in a lamellar structure of dual phase steel. 


\subsubsection{Intercritical annealing and reversion (NLQ) treatment}

A reversion $(\mathrm{Q})$ treatment is given to the alloy after an L-treatment. Fig. 3-11 showed the resulting microstructure after oil quenching, chilling at $77 \mathrm{~K}$ for 1 hour and tempering at 755K for 5 hours. The selected Area diffraction pattern in Fig. 3-11 revealed that there are actually two diffraction patterns within one packet, one corresponding to Kurdjumov-

Sachs (K-S) relationship, ${ }^{[69]}$ the other to Nishiyama-Wasserman $(\mathrm{N}-\mathrm{W})$ relationship ${ }^{[70,71]}$ with the parent austenite phase. The co-existence of $\mathrm{K}-\mathrm{S}$ and $\mathrm{N}-\mathrm{W}$ orientation relationships within one packet generates a large angle misorientation $\left(\sim 54.7^{\circ}\right)$ between adjacent laths, yielding an effective grain size of about $0.5 \mu \mathrm{m}$ on $\{100\}$ planes as shown by dark field image in Fig. 3-11. Thus, a combination of L and Q treatment can directly disrupt the crystallographic orientation relationship between adjacent laths.

We shall discuss what is $\mathrm{K}-\mathrm{S}, \mathrm{N}-\mathrm{W}$ relationship, why they will coexist after an LQ treatment and how the coexistence of these two relations will refine effective grain size in lath martensitic steel in details below.

\section{What is $\mathrm{K}-\mathrm{S}$ and $\mathrm{N}-\mathrm{W}$ relationships and why they coexist?}

It is generally believed that in low alloy steels (for example, $\mathrm{Ni} \mathrm{wt}^{\mathrm{O}} \%<28 \%$ in $\mathrm{Fe}-\mathrm{Ni}$ system), the crystal axis of martensite will form a Kurdjumov-Sachs relationship (K-S) with parent austenite phase. These relations are $(111)_{\gamma} / /(011)_{\alpha},[\overline{1} 01]_{\gamma} / /[\overline{1} 11]_{\alpha}$, so that the close packed planes of the $\gamma$ lattice are parallel to those of the $\alpha$ ' lattice and the closed packed directions of the $\gamma$ are parallel to those of the $\alpha^{\prime}$ (Fig. 3-12). ${ }^{[69]}$ On the other hand, in high alloy cases, the orientation relationships between martensite and parent 
austenite are $(111)_{\gamma} / /(011)_{\alpha},[\overline{1} 10]_{r} / /[100]_{\alpha^{\prime}}$, called Nishiyama-Wassermann (N-W) relations (Fig. 3-12). ${ }^{[70,71]}$ In general, there is only one of the $24 \mathrm{~K}-\mathrm{S}$ or $12 \mathrm{~N}-\mathrm{W}$ variants within a packet or block. Not surprisingly, this is the case we observed in AerMet 100 after N, NQ and NL treatment. However, after NLQT treatment, the crystallographic alignment within the packets or blocks was disrupted and multiple diffraction patterns were observed as shown in Fig. 3-11. To understand why and how this occurs, we have to start from intercritical annealing or L treatment.

As shown in Fig. 3-8 and 3-9, an intercritical annealing will form a lamellar structure with solute $(\mathrm{Ni}, \mathrm{C})$-rich fresh martensite and solute-lean tempered martensite alternating with one another. On the heating to the austenite field in Q treatment, both constituents of L-treated steel revert to austenite. If it is held in the $\gamma$-field for some time after reversion if the part is thick plate, the carbon atoms may redistribute, but the compositional inhomogeneity of solute $\mathrm{Ni}$ will be preserved due to the low diffusivity of the substitutional species in the close-packed FCC $\gamma$ phase. On the subsequent cooling, the dual-phase alloy undergoes a two-step martensitic phase transformation. The solute-lean constituent will transform first, and severely deform the surrounding austenite due to the volume change associated with phase transformation. The first transformation will be stopped due to the increase of strain energy and thus the increase of the resistance to transformation and the lack of driving force once the frontier of martensite encounters the high-alloy constituents. Upon further cooling, the high-alloy constituent will then transform. Under severe mechanical deformation and constraints, the local volume of solute-rich constituent is encouraged to transform to the martensitic variants that are most 
compatible with the local stress field, rather than those that continue the pattern in the surrounding martensite. Therefore, the crystallographic alignment within a packet has been disrupted and there are different orientation relationships (K-S and N-W) with parent austenite between adjacent laths as a result of two-step martensitic transformation.

\section{Two-step martensitic transformation}

The two-step martensitic transformation can be observed through dilatometer test, which measures the volume/length change of alloy sample with temperature during heating and cooling. Since martensitic transformation is associated with volume change, this method has been widely used to measure the $\mathrm{M}_{\mathrm{s}}$ temperature (martensitic transformation starting temperature). Fig. 3-13 showed the dilatometer curve of AerMet 100 samples after (a) Normalization treatment $\left(\mathrm{N}, 1150^{\circ} \mathrm{C}\right.$ for $\left.18 \mathrm{hrs}\right)$ and (b) Normalization and Intercritical Annealing treatments (NL, $1150^{\circ} \mathrm{C}$ for $18 \mathrm{hrs}$ and $677^{\circ} \mathrm{C}$ for $1 \mathrm{hr}$ ). Samples were heated to $900^{\circ} \mathrm{C}$, held for 5 minutes and then cooled down. During heating, the sample after the NL treatment has two constituents with different compositions and shows a two-step reverse-martensitic transformation with austenization temperature at about $590^{\circ} \mathrm{C}$ and $710^{\circ} \mathrm{C}$. However, the sample after the $\mathrm{N}$ treatment exhibits only one austenization point at $650^{\circ} \mathrm{C}$. During cooling, the martensitic transformation of the sample after the $\mathrm{N}$ treatment started at $130^{\circ} \mathrm{C}$ and finished around $60^{\circ} \mathrm{C}$, while the sample after the $\mathrm{NL}$ treatment has a martensitic transformation from $270^{\circ} \mathrm{C}$ to $160^{\circ} \mathrm{C}$. It has to be noted that the martensitic transformation in the latter does not seem to be completed since the length change is still in the negative region, indicating there is still a significant amount of 
austenite remaining in the sample. Therefore, it seems that the constituent with low Ms temperature did not transform yet.

The difference in Ms for Ni-rich and Ni-lean constitutes in this microstructure can be estimated according to the equation Picking gave: ${ }^{[72]}$

$$
\Delta M_{s}\left({ }^{\circ} C\right)=-450 N \%-450 C \%-20 N i \%+10 C o \%-35 C u \%-30 M n \% \ldots
$$

where the percentages are in weight. Although the carbon concentration may have been redistributed evenly in the reversion heat treatment, the compositional profiles of $\mathrm{Ni}$ $(\sim 10 \%$ difference $)$ and Co ( $\sim 5 \%$ difference $)$ remain unchanged due to their low diffusivities in a close-packed (FCC) austenite structure. Hence the difference in Ms temperature $\left(\Delta \mathrm{M}_{\mathrm{s}}\right)$ can be estimated accordingly to be about $250^{\circ} \mathrm{C}$. Therefore, because the first Ms temperature is about $260^{\circ} \mathrm{C}$ (shown in Fig. 3-13), it is very likely that the Ms temperature of second step transformation is just below room temperature, making it very difficult to be observed through dilatometer test unless liquid Nitrogen cooling is used. However, in reality, chilling at $77 \mathrm{~K}$ was performed right after quenching to make sure that the second step martensitic transformation is completed. Thus, it is reasonable to assume that there is a two-step martensitic transformation during the cooling and as a consequence, the crystallographic orientation relationship within a packet has been disrupted.

\section{Impacts on grain refinement and the practical approaches}

The coexistence of K-S and N-W orientation relationships between adjacent laths within one packet is a very interesting phenomenon. On one hand, as shown in Fig. 3-12, there is 
only very small misfit $\left(\sim 5^{\circ}\right.$ in plane rotation) at the lath boundary plane between two adjacent laths, indicating at least an semi-coherent interface with very low interface energy between adjacent laths. On the other hand, this small twist of atoms in lath boundaries creates a large angle $\left(\sim 53^{\circ}\right.$ between $(001)$ planes for $\mathrm{N}-\mathrm{W}$ and for K-S relationships) misorientation between adjacent lath. Because of this large angle misorientation, as shown in far right of Fig. 3-12, if two adjacent martensite crystals have K-S and N-W relationship with parent austenite respectively, they can not share the same $\{001\}$ cleavage plane. Therefore, cleavage propagation will be stopped at lath boundaries and forced to diverge to the nearest cleavage plane in the adjoining lath that is oriented at a finite twist angle from the current cleavage plane. Thus, the effective grain size, or, the coherent length of $\{100\}$ cleavage plane is the cross-section size of the martensitic length that is in the sub-micron regime. As a result, the toughness at low temperature is improved by this grain refinement treatment without sacrifice of strength.

Now the question left to us is why the commercial heat treatments of AerMet 100 were so thoroughly grain-refined with submicron effective grain size. As discussed before, the QT treatment specified for the alloy would not have accomplished this. However, reference to the Carpenter data sheet for AerMet $100^{[62]}$ revealed that the alloy is given a "stress-relief" treatment after forging and prior to shipment, whose intent is to soften the alloy and make it more machineable. The stress-relief treatment is described as " 1200 $1250^{\circ} \mathrm{F}$ (i.e. $649-677^{\circ} \mathrm{C}$ ) for 4 to 16 hours". Since this temperature range falls into the upper part of the two-phase region, this stress-relief treatment is essentially an intercritical annealing, which prepares the alloy for the following grain refinement with a 
complete LQT treatment from as-forged condition, and thus, produces a submicron effective grain size. This observation tells us another advantage of LQT grain refinement techniques. Since stress-relief treatment is a necessary step after forging, especially for those high strength steels, it can really serve two purposes if appropriate temperature and time are used - one for stress relief, the other for intercritical annealing. Therefore, with the following Q treatment that is always included in the processing of the lath martensitic steels, this grain refinement treatment can be completed without extra cost and time for additional heat treatment, an advantage most attractive to the steel industry for practical applications. 


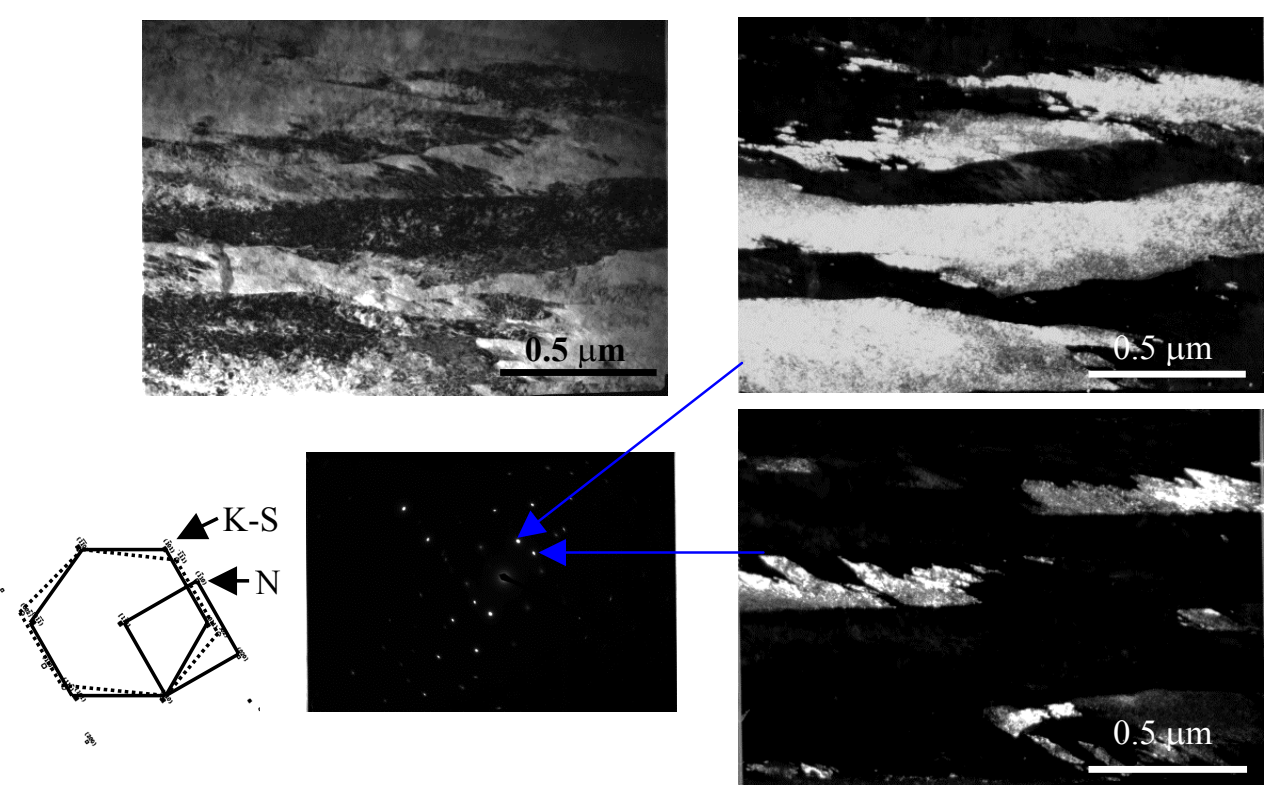

Fig. 3-11 TEM microstructure of AerMet 100 in the NLQT condition (1423K, 18hrs for normalization; $950 \mathrm{~K}, 1 \mathrm{hr}$ for intercritical annealing; $1158 \mathrm{~K}, 1 \mathrm{hr}$, oil quenched; $77 \mathrm{~K}, 1 \mathrm{hr}$ for chilling, $755 \mathrm{~K}$, $5 \mathrm{hrs}$ for tempering). Local diffraction pattern indicates that adjacent laths have different variants in the same martensite packet. 


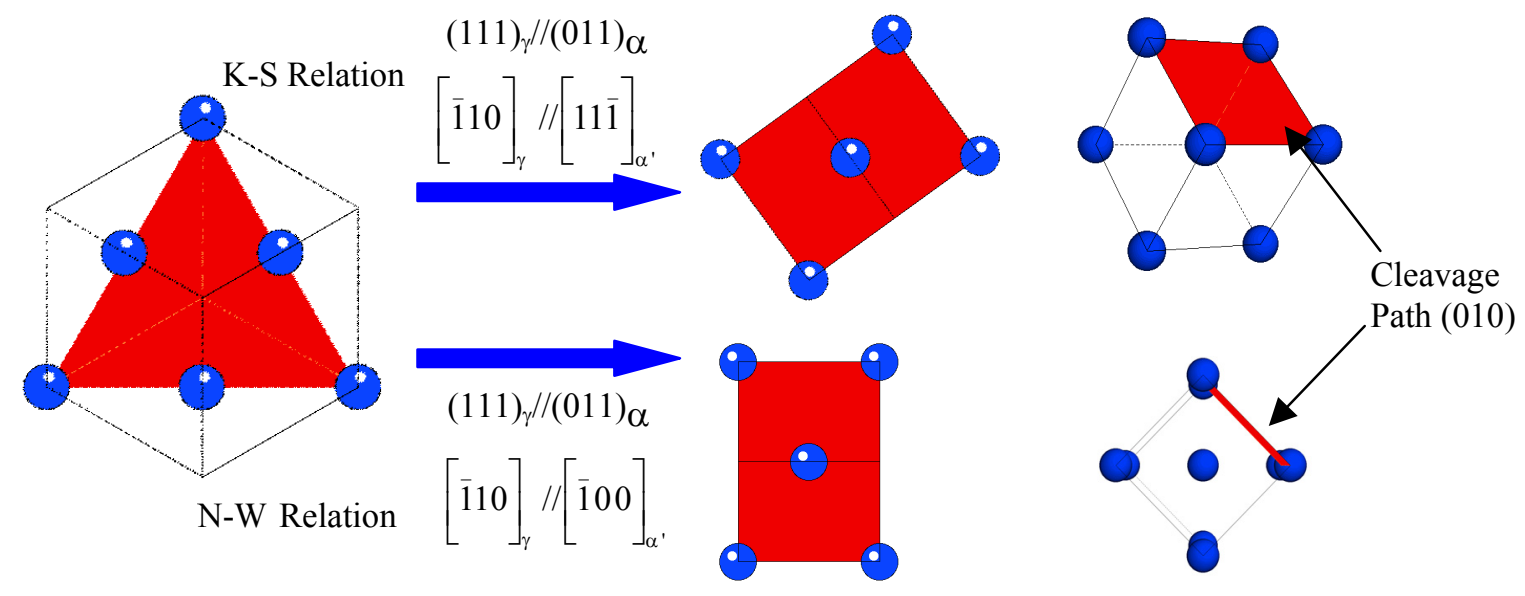

Fig. 3-12 Crystallographic illustration of K-S and N-W orientation relationships between martensite and austenite, indicating that there are small misfit but large misorientation on the boundaries between two adjacent laths if they have K-S and N-W relations with parent austenite phase. As a result, the cleavage propagation will be stopped and diverged at the lath boundaries. 


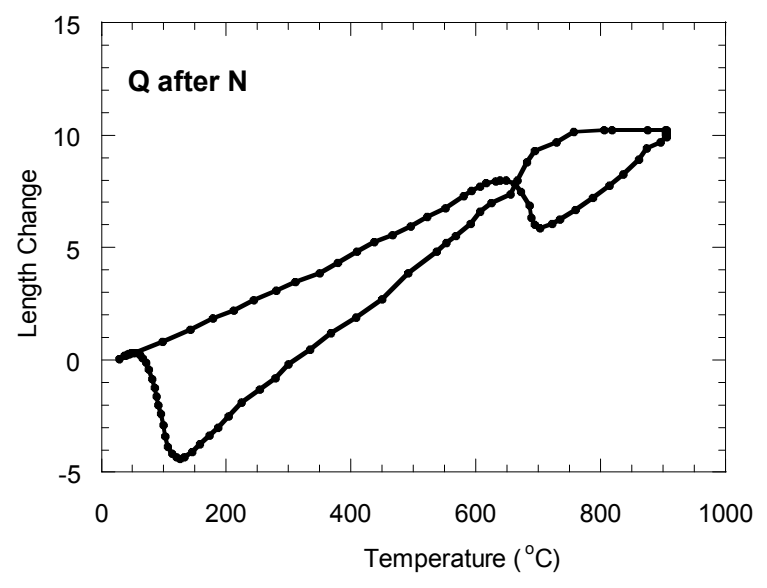

(a)

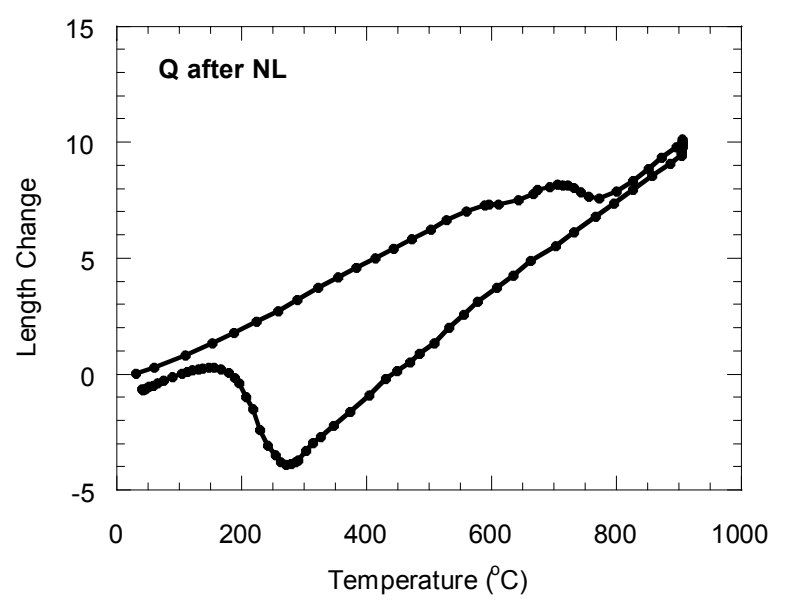

(b)

Fig. 3-13 Dilatometric measurement of AerMet 100 samples after (a) Normalization (N) treatment $\left(1150^{\circ} \mathrm{C}\right.$ for $\left.18 \mathrm{hrs}\right)$ and (b) Normalization (N) and Intercritical Annealing (L) treatment $\left(1150^{\circ} \mathrm{C}\right.$ for $18 \mathrm{hrs}$ and $677^{\circ} \mathrm{C}$ for $\left.1 \mathrm{hr}\right)$. Samples are heated to $900^{\circ} \mathrm{C}$ and then cooled down. 


\subsubsection{Microstructure observation in 9Ni steel}

Similar results have been observed in 9Ni steel. Fig. 3-14 shows the microstructure of 9Ni steel after a NQ treatment. The clean diffraction pattern taken from the interior of the whole packet and the dark field image both indicate that there is only one variant in martensite packet. It proves that austenite reversion (Q) treatment itself can not break crystallographic alignment within the same martensite packet.

However, after an LQ treatment, multiple variants are observed again within the same packet of 9Ni steel, just like in AerMet 100. Fig. 3-15 shows the microstructure of 9Ni steel after an LQ treatment, indicating the coexistence of $\mathrm{K}-\mathrm{S}$ and $\mathrm{N}-\mathrm{W}$ relations in alternated lath and thus the disruption of crystallographic alignment within the packets. This also proves that the LQ treatment, which refines grain size directly, can also be applied to other steels. 


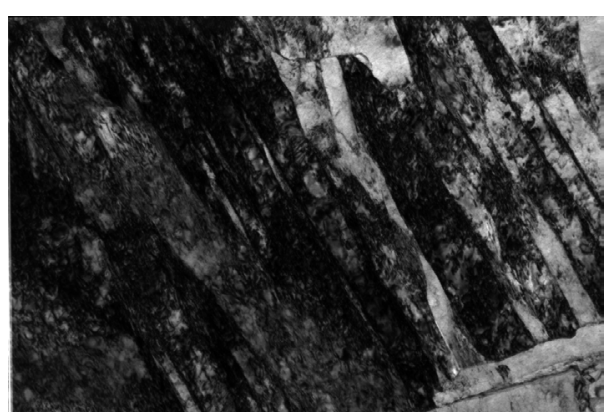

(a)

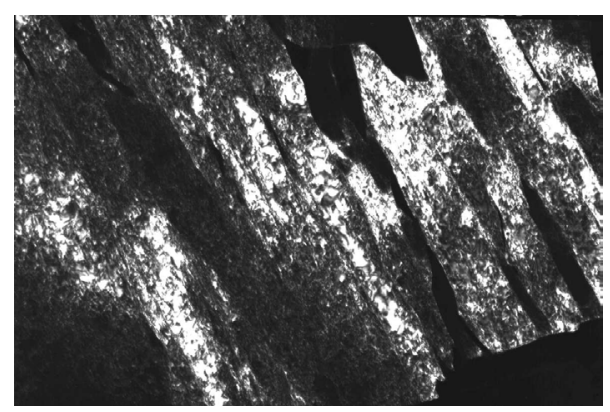

(b)

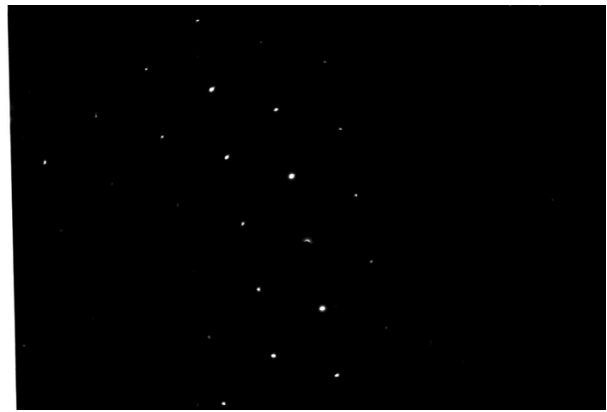

(c)

Fig. 3-14 TEM Bright field, dark field microstructure, and diffraction pattern for 9Ni Steel in the NQ condition (1423K, 18hrs for normalization; 1093K 1hr oil quenched; 77K $1 \mathrm{hr}$ for chilling), showing that good crystallographic alignment remains in martensite packets. 


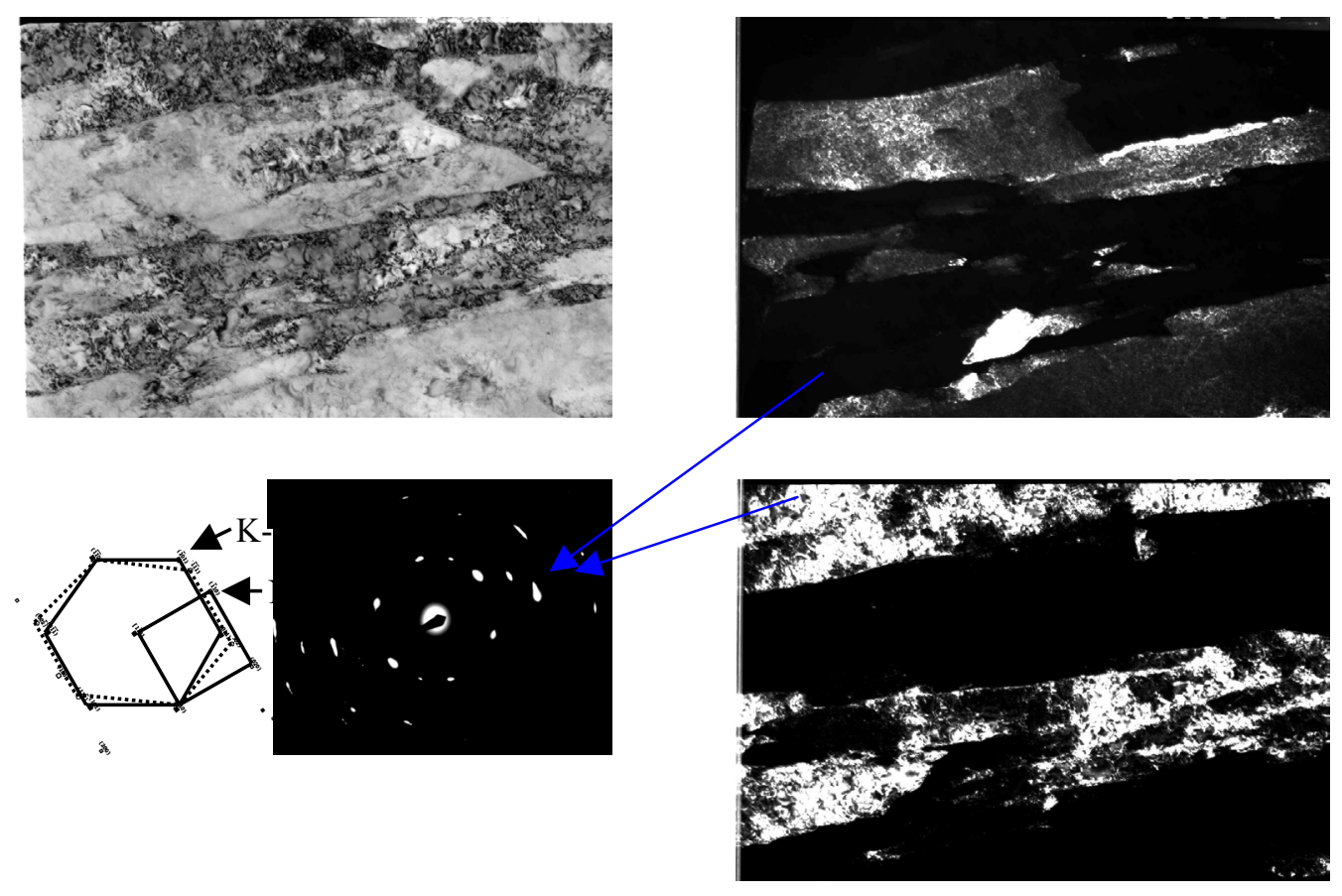

Fig. 3-15 TEM micrographs of 9Ni steel in the NLQT condition (1423K, 18hrs for normalization; $963 \mathrm{~K}, 1 \mathrm{hr}$ for intercritical annealing; $1093 \mathrm{~K}, 1 \mathrm{hr}$, oil quenched; $77 \mathrm{~K}, 1 \mathrm{hr}$ for chilling). Local diffraction pattern indicates two variants within the same martensite packet. 


\subsection{Mechanical Properties}

In order to investigate how this grain refinement affects the alloy's fracture behavior, especially transgranular cleavage fracture, a series of mechanical tests has been conducted.

First, AerMet 100 were heat treated under NT, NQT, NLQT, NLQLQT conditions according to the definition discussed in Section 3.3, then charpy and $\mathrm{J}$ tests were conducted at $77 \mathrm{~K}$. The reason that the NLQLQT condition was added was that previous research in our group has proven that cyclical heat treatment can usually refine grain size more thoroughly. As listed in Table 3-1, both impact energy and $\mathrm{K}_{\mathrm{Q}}$ value increase with the decrease of the effective grain size from NT, NQT condition to NLQT and NLQLQT condition. Fractography of those samples in Fig. 3-16 showed that cleavage size decreases significantly from about $50 \mu \mathrm{m}$ in NT condition to about $7 \mu \mathrm{m}$ in NLQLQT condition.

Table 3-1 Results of charpy and $\mathrm{J}$ tests at $77 \mathrm{~K}$ for different heat treatments

\begin{tabular}{|c|c|c|c|}
\hline Heat Treatment & Impact Energy $(\mathrm{ft \bullet} / \mathrm{b})$ & $\mathrm{K}_{\mathrm{Q}}(\mathrm{MPa} \bullet \sqrt{\mathrm{m}})^{*}$ & Cleavage Size \\
\hline NT & 5.47 & $\mathrm{~N} / \mathrm{A}$ & $\sim 50 \mu \mathrm{m}$ \\
\hline NQT & 9.49 & 30.7 & $\sim 20 \mu \mathrm{m}$ \\
\hline NLQT & N/A & 43.1 & $\sim 10 \mu \mathrm{m}$ \\
\hline NLQLQT & 14.57 & 62.2 & $\sim 7 \mu \mathrm{m}$ \\
\hline
\end{tabular}

* $\mathrm{K}_{\mathrm{Q}}$ value is only for comparison purposes since sample is too thin to achieve plane strain condition 

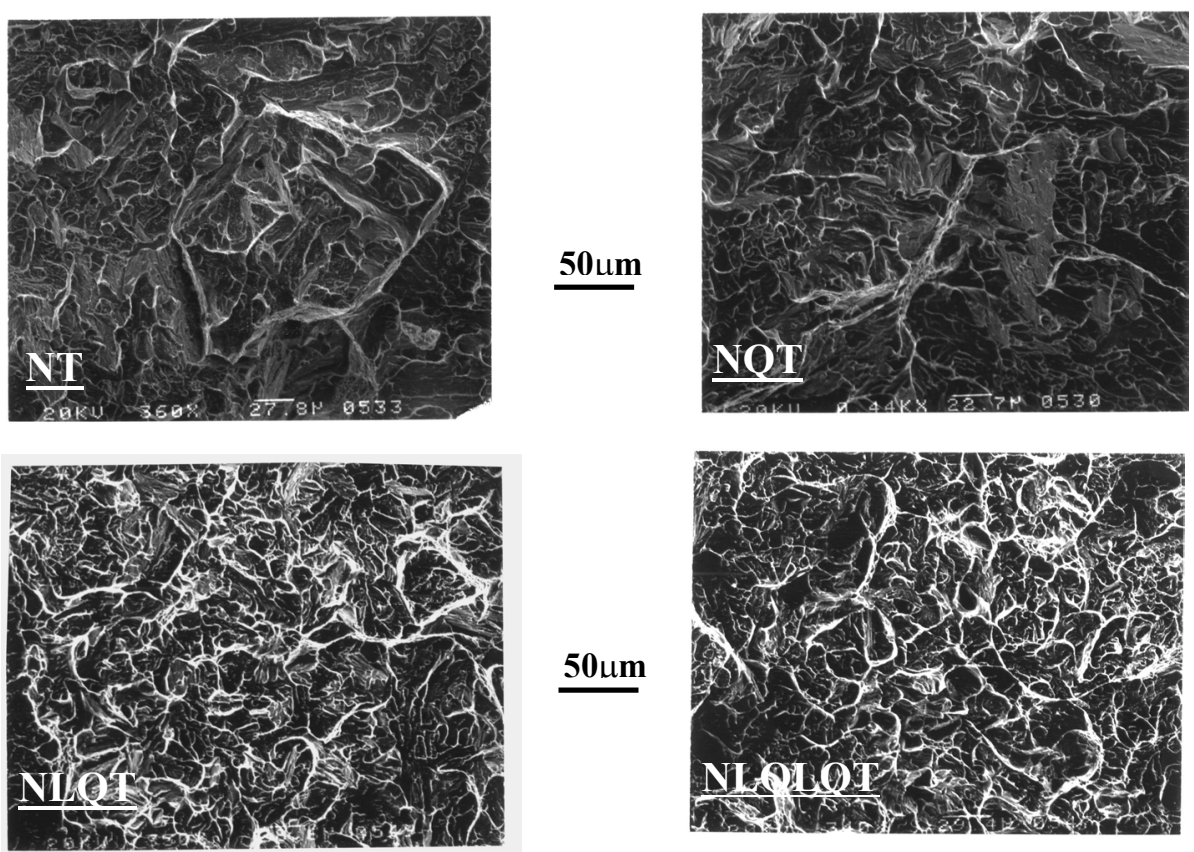

Fig. 3-16 Fractography of $\mathrm{J}$ specimens of AerMet 100 with different heat treatments tested at $77 \mathrm{~K}$, indicating a decrease of cleavage size from NT, NQT to NLQT and NLQLQT. 
Secondly, uniaxial tension and $\mathrm{J}_{\mathrm{IC}}$ tests were performed at both room temperature and 77K for AerMet 100 treated under QT, LQT, and LQLQT conditions. At room temperature, all samples failed in ductile mode with similar $\mathrm{K}_{\mathrm{IC}}$ value, indicating that the grain refinement has little, if any, effect on the ductile fracture, which is determined by void nucleation and coalescence. However, as listed in Table 3-2, at low temperature (77K), toughness increases from $26 \mathrm{MPa}^{*} \mathrm{~m}^{0.5}$ to $33 \mathrm{MPa}^{*} \mathrm{~m}^{0.5}$, (about $20 \%$ increase) from QT condition to LQLQT condition without any loss on strength. In fact, both yielding strength and ultimate tensile strength also increased with the decrease in the effective grain size. Fig. 3-17 shows the fractography of AerMet 100 specimen for different heat treatment conditions tested at $77 \mathrm{~K}$. It is apparent that the cleavage size decreases from about $10 \mu \mathrm{m}$ in QT condition to 3-4 $\mu \mathrm{m}$ in LQLQT condition. In addition to fractography and toughness value, the effect of the decrease of effective grain size can also be observed in the increase of resistance to the crack propagation along cleavage plane as shown in Fig. 3-18. While the specimen with QT treatment showed a large propagation at the very beginning of crack initiation (Fig. 3-18 (a)) and several rapid propagation (abrupt load drop) afterwards (Fig. 3-18 (b)), those with refined effective grain size (LQLQT) exhibit a very smooth curve for both load vs. strain and J integral vs. crack length change, indicating a much more stable crack growth.

Table 3-2 Results of uniaxial tensile and $\mathrm{J}$ tests at $77 \mathrm{~K}$ with different heat treatments

\begin{tabular}{|c|c|c|c|}
\hline Heat Treatment & Yielding Strength (MPa) & Tensile Strength (MPa) & $\mathrm{K}_{\mathrm{IC}}\left(\mathrm{MPa}^{*}{ }_{\mathrm{m}}\right)$ \\
\hline QT & 2066 & 2474 & 27.4 \\
\hline LQT & 2062 & 2442 & 27.6 \\
\hline LQLQT & 2135 & 2494 & 33.1 \\
\hline
\end{tabular}




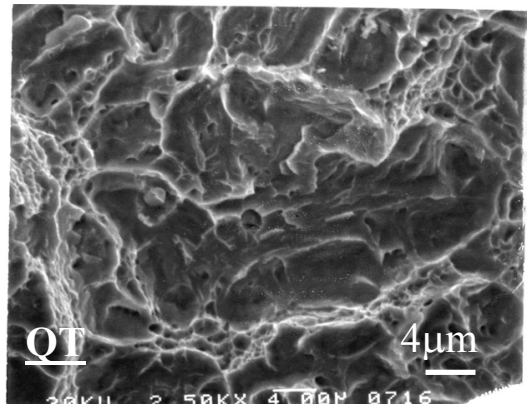

(a)

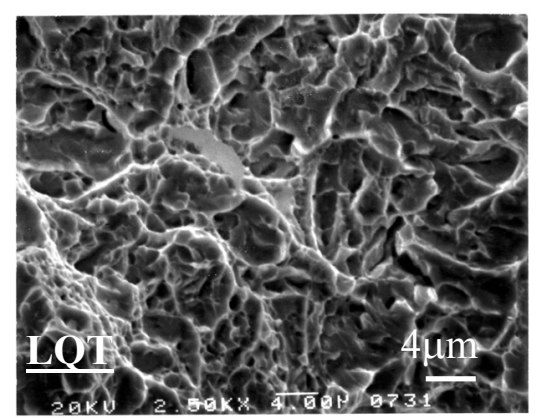

(b)

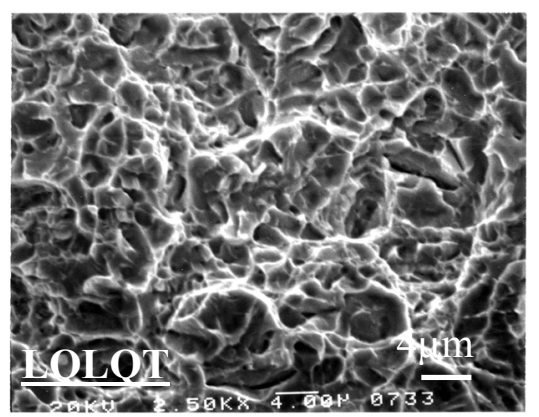

(c)

Fig. 3-17 Fractography of J specimen of AerMet 100 with different heat treatment tested at $77 \mathrm{~K}$, indicating a decrease of cleavage size from about $20 \mu \mathrm{m}$ in QT to $6 \mu \mathrm{m}$ in LQT and $3 \mu \mathrm{m}$ in LQLQT condition. 


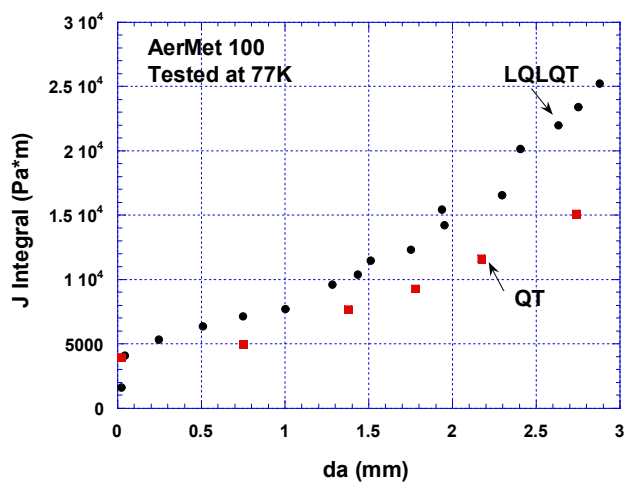

(a)

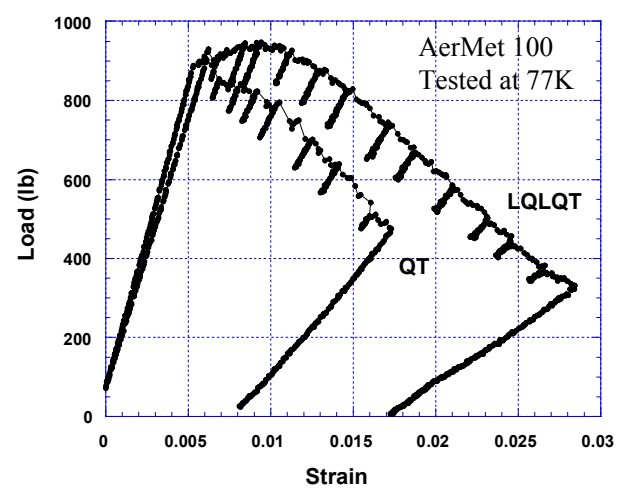

(b)

Fig. 3-18 J Testing results for AerMet 100 at 77K (a) J integral vs. da and (b) Load vs. strain curve, showing that grain refinement can stabilize crack propagation. 


\subsection{Conclusions}

An LQ treatment (an intercritical annealing followed by austenite reversion and quenching) is an effective method to achieve submicron effective grain size by disrupting the crystallographic alignment within the same packet. It accomplishes this because the $\mathrm{L}$ treatment creates a fine-scale lamellar "dual-phase" structure with two different compositions. When this compositionally inhomogeneous structure is austenized and quenched (the $\mathrm{Q}$ treatment), it re-transforms to martensite in a two-step martensitic transformation. These two constituents will severely deformed and constrained each other, disrupting the crystallographic alignment within the same packet and generating large angle misorientation between adjacent laths. Therefore, the crack propagation will be stopped and diverged at lath boundaries, resulting in a submicron effective grain size and a higher resistance to the transgranular cleavage fracture. 


\section{Chapter IV. Martensitic Theory and Grain Refinement}

Chapter III focused on the experimental measures of grain refinement in high strength lath martensitic steel achieved through the disruption of the crystallographic alignment within a packet. Both K-S and N-W relationships were observed in alternating laths within a packet after an LQ treatment, resulting in a sub-micron effective grain size in terms of brittle cleavage fracture. In this chapter, we will explain why the co-existence of these two orientation relationships is energetically favorable and under which circumstances it will occur. We will also discuss whether having different orientation relationships between the martensite laths and the austenite matrix is a sufficient condition for refinement of effective grain size, and to what extent the elastic strain energy plays a role in the grain refinement of high strength steels.

\section{$\underline{4.1 \quad \text { Introduction }}$}

Martensitic transformation, as defined by Cohen, Olson and Clapp, ${ }^{[73]}$ is considered to be a first-order solid-state structural change which is (a) displacive, (b) diffusionless, and (c) dominated in kinetics and morphology by the strain energy arising from shear-like displacement. During the martensitic transformation, the rearrangement of the crystal lattice proceeds through a cooperative movement of atoms, meaning that atomic neighbors are maintained before and after the diffusionless transformation. Hence, this transformation will generally produce a product phase that has some particular orientation relationship with its parent phase. On the other hand, the martensitic 
transformation induces a lattice rearrangement, which, in turn, leads to a large amount of elastic strain. The accommodation of the elastic strain will determine the kinetics and morphology of the transformation. As a result, the martensite phase grows in a pattern that keeps the elastic energy at a minimum or at least a tolerable level. Since the discovery of "martensite" in the late $19^{\text {th }}$ century, there have been two parallel approaches to the study of the martensitic transformation: crystallographic theory or linear elasticity theory.

\subsubsection{Bain correspondence and distortion}

In 1924, Bain first studied the crystallography of martensite and proposed a possible mechanism for the deformation of austenite into the martensite lattice. ${ }^{[74]}$ Fig. $4-1$ shows this model, in which a BCC lattice can be obtained from an FCC lattice by compression along one principle axis, e.g. $[100]_{\mathrm{FCC}}$, and a simultaneous uniform expansion along the two axes which are perpendicular to it. This homogenous lattice deformation is called the Bain Distortion and the correspondence between the lattice points in the initial and final lattices can be determined uniquely, known as the Bain Correspondence. One can find other possible lattice deformations to generate a BCC (BCT) lattice from a FCC lattice. However, the Bain Deformation is the most reasonable one because it involves the smallest relative atomic displacement and thus the lowest strain energy. Bain distortion is an essential ingredient of both the crystallographic theory and the linear elasticity theory. 


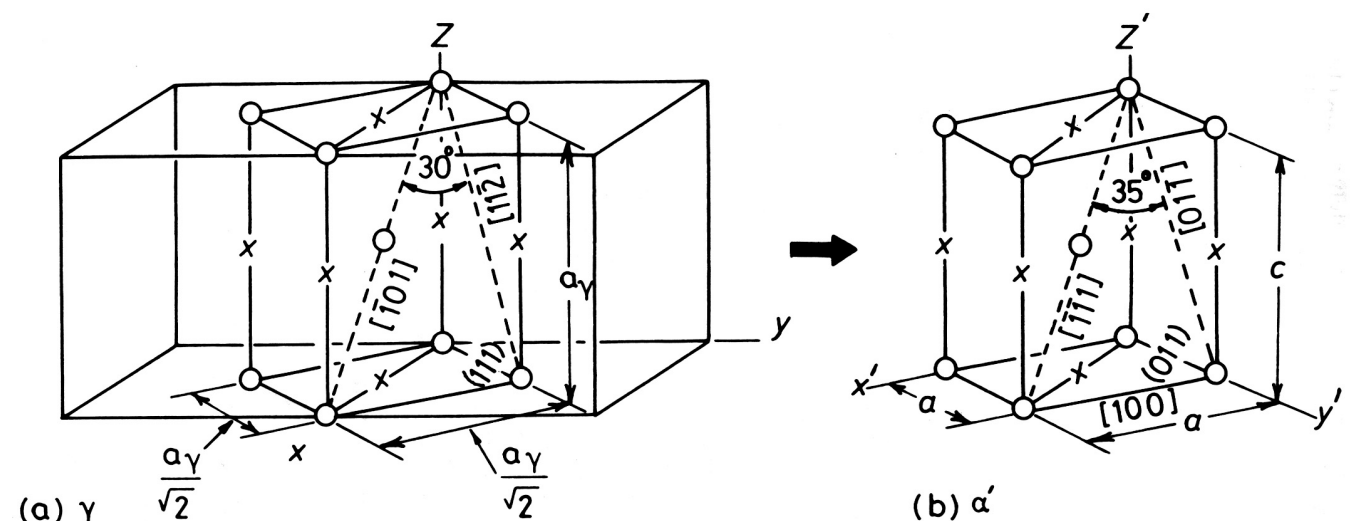

Fig. 4-1 Bain correspondence in the martensitic transformation of FCC to BCC or BCT

\subsubsection{Shear mechanism models for the martensitic transformation}

The Bain distortion considers only the correspondence between the final and initial lattice; it does not give us the actual crystal orientation relationships, such as K-S and N$\mathrm{W}$ relationships, between the parent austenite and product martensite. The shear mechanism, on the other hand, relates them through a shear on the planes and along the directions involved in the orientation relationships. As shown in Fig. 4-2 (b), martensites with a K-S relationship are thought to be formed from an austenite parent phase by a shear on $\{111\}_{\gamma}$ planes along $\langle 110\rangle_{\gamma}$ directions, while those with a N-W relationship are considered to be generated by a shear on $\{111\}_{\gamma}$ planes along $<112>_{\gamma}$ directions. ${ }^{[69,70]}$ In addition, another shear out of the $\{111\} \gamma$ is necessary in order to maintain the correct stacking sequence of the close-packed planes for both BCC and FCC (Fig. 4-2 (a)). Both of these shear mechanisms are identical to the Bain Distortion if the rigid body rotation of martensite due to shear is disregarded. Therefore, both mechanisms have been considered as reasonable models for a long time. 


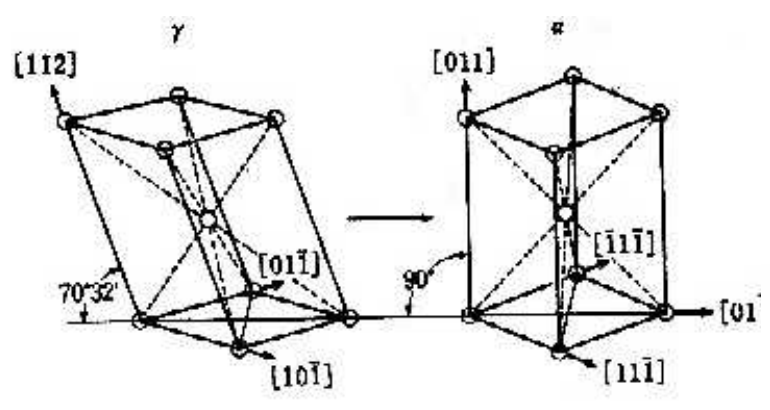

(a)

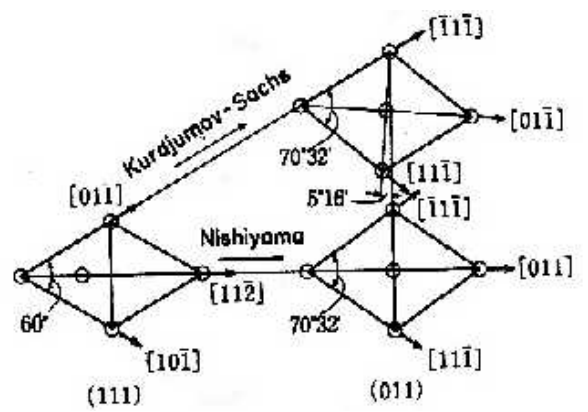

(b)

Fig. 4-2 Illustrations of shear mechanisms (a) outside and (b) within close-packed planes in the $\gamma \rightarrow \alpha^{\prime}$ transformation proposed by Kudjumov and Sachs ${ }^{[69]}$ and Nishiyama ${ }^{[70]}$.

\subsubsection{Double shear mechanism - crystallographic phenomenological theory}

As experimental results have accumulated, the simple shear mechanisms have been found to be too simplistic and not consistent with all of the experimental findings. For example, since the martensitic transformation proceeds through a cooperative motion of atoms, the interface between the parent and product phases must be highly coherent. Therefore, during the transformation, the interface should be an unrotated and undistorted plane, called an invariant plane. In general, if an undistorted plane is to result from a homogeneous deformation (called invariant plane strain), one of the principal distortions must be greater than unity, one must be less than unity and the third one equal to unity. ${ }^{[75]}$ However, it is clear that the Bain Distortion has no invariant plane. As shown in Fig. 4-3 (a), the Bain distortion will deform a unit sphere representing the parent phase to an oblate spheroid representing the product phase. The vectors that initially composed the cones $\mathrm{AOB}$ and $\mathrm{COD}$ are now transformed to those composing the cones $\mathrm{A}^{\prime} \mathrm{OB}^{\prime}$ and C'OD' defined by intersections of the unit sphere with the spheroid. These vectors are termed as unextended lines since their magnitude remains unchanged during the lattice 
deformation. However, there exists no rigid body rotation that can rotate two unextended lines simultaneously back to their original positions while keeping the angle between them unchanged. Therefore, the Bain distortion does not results in an invariant plane. Since both K-S and N-W relationships are just rigid body rotations of the Bain distortion, they will not produce an invariant plane strain either.

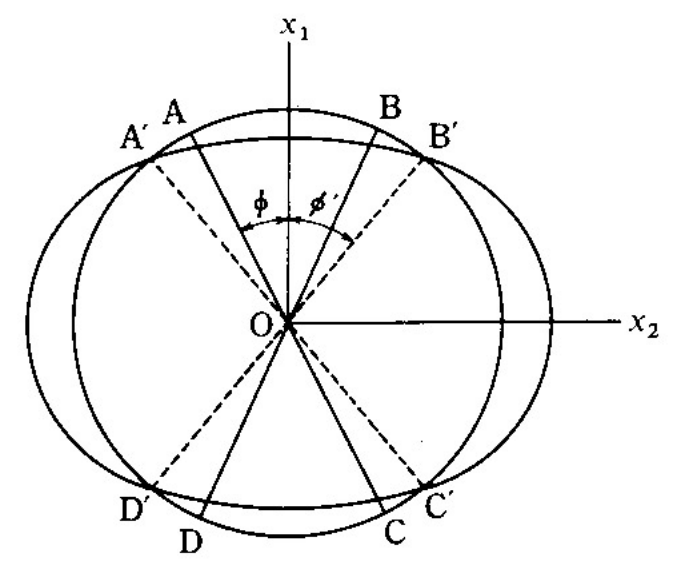

(a)

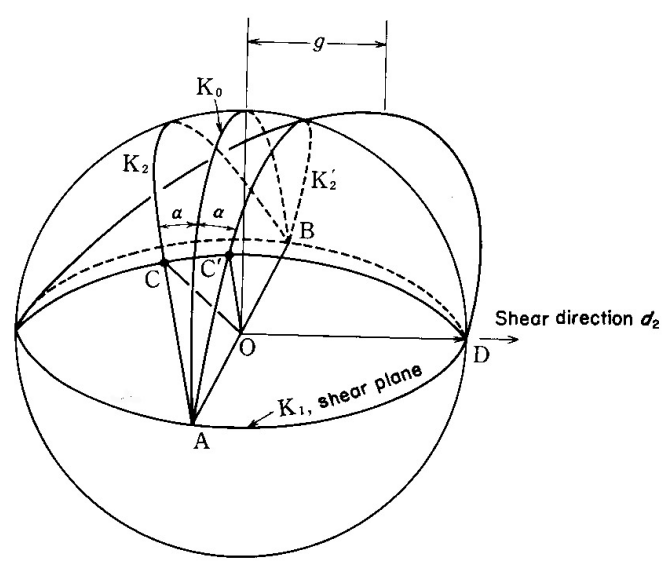

(b)

Fig. 4-3 Illustration of (a) Bain Distortion has no invariant plane and (b) a second shear can generate a lattice invariant shear.

In order to overcome this difficulty, it is necessary to introduce a second shear. Thus, the crystallographic theory of martensitic transformation was developed in the late 50's independently by Bowles and Mackenzie (B-M theory) ${ }^{[76]}$ and by Wechsler, Liberman and Read (W-L-R theory) ${ }^{[77]}$. As shown in Fig. 4-3 (b), the whole purpose of introducing this additional shear is that the spheroid resulting from Bain Distortion is sheared into tangency with the initial unit sphere. In addition, this second shear should not bring about any change in crystal structure and thus is a lattice invariant shear. Therefore, the 
martensitic transformation is essentially considered to consist of a lattice deformation or Bain Distortion (B), a lattice invariant shear $(\mathbf{P})$ and lattice rotation $(\mathbf{R})$ which is needed in order to rotate the invariant plane back to its original position. Generally speaking, the lattice invariant shear can be accomplished by either dislocation slip in dislocated laths or by mechanical twining in twinned plates. It has to be pointed that both crystallographic orientation and elastic strain are only determined by the Bain distortion (B) and the lattice rotation $(\mathrm{R})$, since the second shear $(\mathrm{P})$ does not change either the crystal orientation or the elastic strain.

This phenomelogical crystallographic theory has been particularly successful in predicting the habit plane and and the volume fraction of twinned martensite in steels and other alloys. ${ }^{[78]}$ Despite its success, the crystallographic theory does not provide the direct linkage of the strain energy to the transformation kinetics, nor to the morphology of the martensitic transformation. For example, it cannot explain why and how the packets or blocks are formed.

\subsubsection{Linear elasticity theory of martensitic transformation}

Emerging at the end of the 1970 's, linear elasticity theory provided a new approach that directly relates the morphology of martensite to the elastic strain energy induced during the transformation. Based on a solution developed by Eshelby ${ }^{[79,80]}$ and extended by Khachaturyan, ${ }^{[81,82]}$ this theory enables us to compute the strain energy for a particle of any shape and with any arbitary distribution. To study the development of microstructure during the martensitic transformation, a computer model has been first proposed by Wen, 
Khachaturyan and Morris ${ }^{[83]}$ using point approxiamation and later modified by Xu and Morris ${ }^{[84,85,86]}$ using element cells. The fundamental basis of these calculations relies on the fact that in some solid state transformations, such as coherent precipitations and martensitic transformations, new phases remain coherent with the matrix. The tendency towards decreasing elastic energy plays an essential role in the formation of the final multiphase structure. However, there are some limitations in the linear elasticity theory since this theory starts from the Bain deformation and ignores the rotational component of the finite strain.

In this chapter, we will try to combine both the crystallographic theory and the linear elasticity theory, and to investigate the orientation relationship as well as the morphology of the product phase during martensitic transformation. We shall first calculate the elastic strain of martensitic transformation for different orientation relations, and then use elastic theory to explain why a second shear is necessary and how it will be accomplished. Furthermore, we will examine the relationship between Bain variants and refinement of the effective grain size. Finally, we will discuss in a general framework the role that elastic strain energy plays during a martensitic transformation and how the orientation relationship and morphology of the product phase can be controlled. 


\subsection{Calculations of Elastic Strain}

As discussed before, since both the crystallographic orientation and elastic strain are determined only by the Bain distortion (B) and lattice rotation (R), we shall start our calculations of the elastic strain induced by martensitic transformation from the K-S and $\mathrm{N}-\mathrm{W}$ orientation relationships. There are $24 \mathrm{~K}-\mathrm{S}$ and $12 \mathrm{~N}-\mathrm{W}$ possible orientation relationships resulting from a martensitic transformation. There are, of course, three $<011>$ directions in each of the four $\{111\}$ planes in FCC crystals. Hence, each closepacked direction in a specific close-packed plane can be associated with one N-W and two twin-related K-S relations. Without losing generality, let us assume that the closepacked plane is (111) and close-packed direction is [1 10] in austenite, and calculate the transformation strain matrix for both the N-W and K-S relationships as an example.

\subsubsection{Deformation and strain matrices for N-W relationship using FCC Coordinates}

The deformation matrix of the martensitic transformation will transform three noncoplanar vectors in the initial austenite lattice into a final martensite lattice that has a specific crystallographic orientation relationship with the parent phase. In the case of the $\mathrm{N}-\mathrm{W}$ relationship, the orientation relationship can be assumed to be $(111)_{\gamma} /(011)_{\alpha}$, and $[\overline{1} 10]_{r} / /[\overline{1} 00]_{\alpha^{\prime}}^{N-W}$. Thus, the vectors are $[\overline{1} 10]_{r} / /[\overline{1} 00]_{\alpha^{\prime}}^{N-W}$ and $[11 \overline{2}]_{r} / /[01 \overline{1}]_{\alpha^{\prime}}^{N-W}$ within the closepacked plane, and $[112]_{\gamma} / /[111]_{\alpha}$, outside the plane in order to maintain the correct stacking sequence of close-packed plane(Fig. 4-4). Here we will use the lattice parameter

of pure iron in $\mathrm{FCC}$ and $\mathrm{BCC}^{[87]}$ and ignore the effect of alloying elements on the lattice 
parameters. Therefore, using three FCC axes as a basis, the deformation matrix and the strain matrix for the N-W relationship can be obtained through the method described by Khachaturyan ${ }^{[82]}$

$$
D e f N W_{F C C}=\left[\begin{array}{ccc}
1.129 & -0.009 & 0.096 \\
-0.009 & 1.129 & 0.096 \\
-0.136 & -0.136 & 0.792
\end{array}\right] \quad \text { Strain } N W_{F C C}=\left[\begin{array}{ccc}
0.137 & -0.0006 & 0.0002 \\
-0.0006 & 0.137 & 0.0002 \\
0.0002 & 0.0002 & -0.196
\end{array}\right] \text { (4-1) }
$$

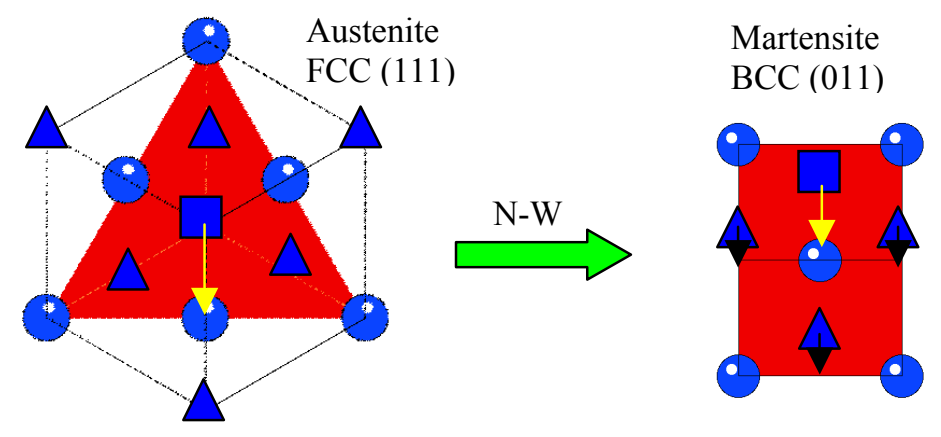

Fig. 4-4 Illustration of crystallographic relationship between initial austenite (FCC) and final martensite (BCC) in an N-W related martensitic transformation.

\subsubsection{Deformation and strain matrices for K-S relationship using FCC Coordinates}

In the case of the K-S relationship, as shown in Fig. 4-5, the close-packed direction in FCC is parallel to the close-packed direction in BCC, i.e. $[\overline{1} 10]_{\gamma} / /[11 \overline{1}]_{a^{\prime}}$. The other in-plane

vector $[011]_{,}^{1}$ is going to be transformed to $[\overline{1} 00]_{\alpha}$. However, both the magnitude and direction of the second vector will be changed during the transformation. If we assume the final vector $[100]_{\alpha}$, is [uvw] in the original FCC axes, it has to satisfy three criteria: 1)

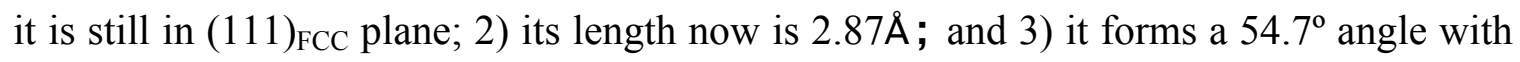


$[11 \overline{1}]_{\alpha^{\prime}}$, i.e. $[\overline{1} 10]_{\gamma}$ direction. Therefore, the final vector in FCC bases is $[-0.12,1.19,-1.07]$.

The out-of-plane shear will transform $[211]_{\gamma}$ into $[111]_{\alpha}$, direction as shown in Fig. 4-5.
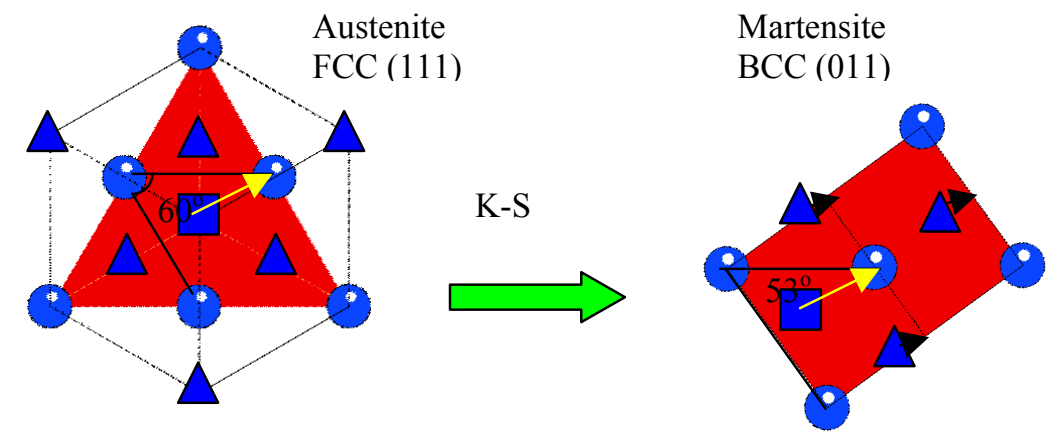

Fig. 4-5 Illustration of crystallographic relationship between initial austenite (FCC) and final martensite $(\mathrm{BCC})$ in a K-S related martensitic transformation.

The deformation matrix can be obtained by considering the initial and final position of these three vectors in the FCC bases. The strain matrix can also be calculated using the same method as for $\mathrm{N}-\mathrm{W}$ relationship.

$$
\operatorname{DefKS1}_{F C C}=\left[\begin{array}{ccc}
0.791 & -0.194 & -0.074 \\
0.134 & 1.119 & -0.074 \\
0.060 & 0.060 & 1.132
\end{array}\right] \quad \text { StrainKS1 }_{F C C}=\left[\begin{array}{ccc}
-0.196 & -0.0002 & -0.0002 \\
-0.0002 & 0.137 & 0.0003 \\
-0.0002 & 0.0003 & 0.137
\end{array}\right]
$$

Using the same method, we can also calculate the strain matrix for the twin-related K-S relations whose orientation relationship is $(111)_{\gamma} / /(011)_{\alpha},[\overline{1} 10]_{\gamma} / /[\overline{1} 1 \overline{1}]_{\alpha^{\prime}}$.

$$
D e f K S 2_{F C C}=\left[\begin{array}{ccc}
1.119 & 0.134 & -0.074 \\
-0.194 & 0.791 & -0.074 \\
0.060 & 0.060 & 1.132
\end{array}\right] \quad \text { StrainKS2 } 2_{F C C}=\left[\begin{array}{ccc}
0.137 & -0.0002 & 0.0003 \\
-0.0002 & -0.196 & -0.0002 \\
0.0003 & -0.0002 & 0.137
\end{array}\right]
$$




\subsubsection{Deformation and strain matrices using BCC Coordinates}

There are two ways to calculate the strain matrix of the martensitic transformation with $\mathrm{N}-\mathrm{W}$ or K-S relationships in BCC coordinates. We can either use the same method described above to calculate it directly, or we can find the transformation matrix that will transform $\mathrm{FCC}$ to $\mathrm{BCC}$ coordinates and then rotate the strain matrix into $\mathrm{BCC}$ axes using linear algebra. These two methods will essentially give us the same results.

Let's consider the transformation matrix in BCC axes for the N-W relationship. Within the FCC (111) plane, or the BCC (011) plane, we have $\left.[\overline{1} 10]_{r} / / \overline{1} 00\right]_{\alpha^{\prime}}^{N-W}$ and $[11 \overline{2}]_{r} / /[01 \overline{1}]_{\alpha^{\prime}}^{N-W}$.

Out of plane, as shown in Fig. 4-4, the atoms in the third layer (marked as square) will have to slide about $1 / 6[11 \overline{2}]$ from its original position. Thus, the original [112] vectors will become $1 / 2[112]+1 / 6[11 \overline{2}]=2 / 3[111]$. This discussion is based on FCC coordinates.

In BCC coordinates, we know that the $[111]_{\gamma}$ direction is $[011]_{\alpha}$, and $[11 \overline{2}]_{\gamma}$ is $[01 \overline{1}]_{\alpha}$, Therefore the original $1 / 2[112]$ vector in BCC coordinates can be expressed as

$$
V_{1 / 2[112]}=2 / 3[111]_{\gamma}-1 / 6[11 \overline{2}]_{\gamma}=\frac{2}{3} \frac{\sqrt{3} a_{f c c}}{\sqrt{2} a_{b c c}}[011]_{\alpha^{\prime}}-\frac{1}{6} \frac{\sqrt{6} a_{f c c}}{\sqrt{2} a_{b c c}}[01 \overline{1}]_{\alpha^{\prime}}=\left(\frac{a_{f c c}}{a_{b c c}}\right)[0,0.528,1.105]_{\alpha^{\prime}}
$$

By solving three equations and using the same method in 4.2.1, we obtain:

$$
\operatorname{DefNW}_{B C C}=\left[\begin{array}{ccc}
1.137 & 0 & 0 \\
0 & 1.121 & 0.192 \\
0 & -0.136 & 0.792
\end{array}\right] \quad \text { StrainN } W_{B C C}=\left[\begin{array}{ccc}
0.137 & 0 & 0 \\
0 & 0.127 & 0.055 \\
0 & 0.055 & -0.186
\end{array}\right]
$$

For the strain matrix of martensitic transformation with a $\mathrm{K}-\mathrm{S}$ relationship in a $\mathrm{BCC}$ coordinate, by applying same method, we will have 
$D e f K S 1_{B C C}=\left[\begin{array}{ccc}1.130 & -0.097 & 0.047 \\ 0.061 & 1.117 & 0.194 \\ -0.061 & -0.133 & 0.791\end{array}\right] \quad$ StrainKS1 $1_{B C C}=\left[\begin{array}{ccc}0.133 & -0.014 & 0.009 \\ -0.014 & 0.128 & 0.055 \\ 0.009 & 0.055 & -0.186\end{array}\right]$

which is very similar to Eqn. 4-5 for the N-W relationship in BCC coordinates.

Thus, the elastic strain matrices for the K-S and N-W relationships in both FCC and BCC coordinates are laid out as a foundation for our further calculations and discussions. 


\subsection{Invariant Plane Strain}

It has been discussed before that an invariant plane strain is a necessary condition for the martensitic transformation because the interface between the parent and product phases has to be highly coherent in order for the transformation to proceed. This is, in fact, the primary incentive for the phenomenological crystallographic theory, such as $\mathrm{B}-\mathrm{M}^{[76]}$ and W-L- $\mathrm{R}^{[77]}$. In this section, we will use the elastic strain energy theory to re-examine this argument.

When a martensitic phase transformation happens in the interior of a parent austenite phase, it perturbs the size, shape and elastic moduli of the transformed volume, resulting in an elastic strain on both the martensite and parent austenite. In our case, let us consider a thin martensitic plate in an infinite parent austenite matrix using the elastic coherent nucleus method and assume it is at the very initial stage of the martensitic transformation. This martensite plate has to be macroscopically small compared to the austenite matrix, but microscopically large in order to apply continuum elastic theory.

\subsubsection{Eshelby Circle for the elastic inclusion problem}

A general approach for elastic inclusion problems was suggested by J. D. Eshelby, who realized that it is always possible to obtain the final state of a constrained elastic inclusion through a sequence of operations called the Eshelby Circle. ${ }^{[79]}$ Consider an elastic solid that is initially homogeneous and unstressed, and let an inclusion of arbitrary shape be introduced by the following sequence of operations: 
1. Isolate the region within a solid that the nucleation will happen, and cut it out. This operation produces no change in the total energy of the system if the interfacial energy is ignored since the stress and strain are zero in both bodies.

2. Let the cut-out volume undergo a stress-free transformation that will involve a change in both size and shape of the inclusion. The inclusion is now still unstressed but has a transformation strain $\varepsilon^{0}$ with respect to the relaxed state of matrix.

3. Impose a set of surface traction on the inclusion and return it to its original size and shape. After this step, the inclusion is unstrained since the strain is measured with respect to the relaxed matrix but has a transformation stress $\sigma^{0}$ that can be calculated from transformation strain $\varepsilon^{0}$ and elastic constant of inclusions $\lambda_{i j k l}$ using Hook's Law

$$
\sigma_{i j}=\sigma_{i j}{ }^{0}=-\lambda_{i j k l} *^{*} \varepsilon_{k l}
$$

4. Re-insert the inclusion into the matrix. Since the inclusion has been returned to its original shape and size, it will just fit into the hole left in the matrix. Therefore, neither the inclusion nor the matrix is strained. However, there is an internal stress field inside the inclusion. The overall stress $\sigma(\boldsymbol{R})$ field can be related to the shape function $\theta(\boldsymbol{R})$ as $\sigma(\boldsymbol{R})=\sigma^{0} \theta(\boldsymbol{R})$ where $\theta(\boldsymbol{R})=1$ inside the inclusion and 0 otherwise. 
5. Remove the surface traction on the inclusion. The internal stress within the inclusion can be relaxed by deforming the inclusion and matrix, which introduces a strain field $\varepsilon(\boldsymbol{R})$. Hence, the associated stress field is

$$
\sigma_{i j}(\boldsymbol{R})=\sigma_{i j}^{0} \theta(\boldsymbol{R})+\lambda_{i j k l} * \varepsilon_{k l}(\boldsymbol{R})
$$

where $\lambda_{i j k l}$ is the elastic modulus for inclusion and $\boldsymbol{R}$ is the set of points within the inclusion phase or in the matrix.

In our case, the macroscopic strain in the infinite parent austenite matrix can be considered to be 0 since the volume fraction of martensite is very small and the surface of austenite matrix is free of traction. Thus, the internal strain relaxation can be calculated by the following:

$$
\varepsilon_{i j}(R)=-1 / 2\left[n_{i} \Omega_{j k}^{e}+n_{j} \Omega_{i k}^{e}\right] \sigma_{k l}^{0} n_{l} \theta(R)
$$

where the Green's Elastic tensor $\Omega^{\mathrm{e}}$ can be expressed as $\left[\Omega^{e}\right]_{i l}^{-1}=\lambda_{i j k l} e_{j} e_{k}$, and $n$ is the habit direction of thin plate. $\sigma^{0}$ is the transformation stress. The final stress field is

$$
\sigma_{i j}(\boldsymbol{R})=\sigma_{i j}{ }^{0}+\lambda_{i j k l} * \varepsilon_{k l}(\boldsymbol{R})=-\lambda_{i j k l} * \varepsilon_{k l}{ }^{0}+\lambda_{i j k l} * \varepsilon_{k l}(\boldsymbol{R})=\lambda_{i j k l}\left(-\varepsilon_{k l}{ }^{0}+\varepsilon_{k l}\right)
$$

within the inclusions and 0 everywhere else in the matrix.

In summary, the key point in solving this problem is to find the relaxed strain field $\varepsilon_{i j}(\boldsymbol{R})$ in Eqn. 4-9. Since we can obtain Green's elastic tensor from the elastic moduli and also know the transformation strain and stress matrix from the last section, this problem is solvable. 


\subsubsection{Stress and strain field calculations}

\section{$\underline{\text { 1. Green's elastic tensor }}$}

Using the crystallographic orientation relationships described in the previous section, we have $(111)_{\gamma} / /(011)_{\alpha}$, for both the K-S and N-W relationships. Therefore, the habit plane is (011) for Martensite. In a cubic system, given $\lambda_{1111}=\lambda_{2222}=\lambda_{3333}=\mathrm{C}_{11}$, $\lambda_{1122}=\lambda_{1133}=\lambda_{2233}=\mathrm{C}_{12}, \lambda_{1212}=\lambda_{2323}=\lambda_{1313}=\mathrm{C}_{12}$, the Green's elastic tensor can be calculated according to Khachaturyan as following: ${ }^{[82]}$

$$
\begin{aligned}
& \Omega_{i i}(n)=\frac{C_{44}+\left(C_{11}-C_{44}\right)\left(n_{j}^{2}+n_{k}^{2}\right)+\xi\left(C_{11}+C_{12}\right) n_{j}^{2} n_{k}^{2}}{C_{44} D(n)} \\
& \Omega_{i j}(n)=\frac{\left(C_{12}+C_{44}\right)\left(1+\xi n_{k}^{2}\right)}{C_{44} D(n)} n_{i} n_{j}
\end{aligned}
$$

where $D(n)=C_{11}+\xi\left(C_{11}+C_{12}\right)\left(n_{1}^{2} n_{2}^{2}+n_{1}^{2} n_{3}^{2}+n_{2}^{2} n_{3}^{2}\right)+\xi^{2}\left(C_{11}+2 C_{12}+C_{44}\right) n_{1}^{2} n_{2}^{2} n_{3}^{2}$

$$
\xi=\frac{C_{11}-C_{12}-2 C_{44}}{C_{44}}
$$

For the (011) habit plane, since $\mathrm{n}_{1}=0, \mathrm{n}_{2}=0.707, \mathrm{n}_{3}=0.707$, we have:

$$
\begin{array}{ll}
\Omega_{11}=1 / C_{44}, & \Omega_{22}=\Omega_{33}=\frac{2\left(C_{11}+C_{44}\right)}{\left(C_{11}-C_{12}\right)\left(C_{11}+C_{12}+2 C_{44}\right)} \\
\Omega_{12}=\Omega_{13}=0, & \Omega_{23}=\frac{-2\left(C_{12}+C_{44}\right)}{\left(C_{11}-C_{12}\right)\left(C_{11}+C_{12}+2 C_{44}\right)}
\end{array}
$$

Thus, we have turned a fourth-rank tensor of elastic constants into a second-rank Green's tensor of elastic moduli along a specific crystallographic direction. 


$$
\left[\begin{array}{cccccc}
C_{11} & C_{12} & C_{12} & 0 & 0 & 0 \\
C_{12} & C_{11} & C_{12} & 0 & 0 & 0 \\
C_{12} & C_{12} & C_{11} & 0 & 0 & 0 \\
0 & 0 & 0 & C_{44} & 0 & 0 \\
0 & 0 & 0 & 0 & C_{44} & 0 \\
0 & 0 & 0 & 0 & 0 & C_{44}
\end{array}\right] \Rightarrow\left[\begin{array}{ccc}
\frac{1}{C_{44}} & 0 & 0 \\
0 & \frac{2\left(C_{11}+C_{44}\right)}{\left(C_{11}-C_{12}\right)\left(C_{11}+C_{12}+2 C_{44}\right)} & \frac{-2\left(C_{12}+C_{44}\right)}{\left(C_{11}-C_{12}\right)\left(C_{11}+C_{12}+2 C_{44}\right)} \\
0 & \frac{-2\left(C_{12}+C_{44}\right)}{\left(C_{11}-C_{12}\right)\left(C_{11}+C_{12}+2 C_{44}\right)} & \frac{2\left(C_{11}+C_{44}\right)}{\left(C_{11}-C_{12}\right)\left(C_{11}+C_{12}+2 C_{44}\right)}
\end{array}\right]_{(011)}
$$

For BCC iron, $\mathrm{C}_{11}=229 \mathrm{GPa}, \mathrm{C}_{12}=134 \mathrm{GPa}, \mathrm{C}_{44}=115 \mathrm{GPa}$ according to Ledbetter, ${ }^{[34]}$ thus

$$
\Omega_{011}=\left[\begin{array}{ccc}
0.0087 & 0 & 0 \\
0 & 0.0122 & 0.0088 \\
0 & 0.0088 & 0.0122
\end{array}\right] \mathrm{GPa}^{-1}
$$

\section{Transformation strain and stress}

The transformation strain in BCC coordinates for both the K-S and N-W relationships has been calculated from Eqn. 4-4 and 4-5 in the previous section as the following:

$$
\text { StrainKS } 1_{B C C}^{0}=\left[\begin{array}{ccc}
0.133 & -0.014 & 0.009 \\
-0.014 & 0.128 & 0.055 \\
0.009 & 0.055 & -0.186
\end{array}\right] \quad \text { StrainNW } W_{B C C}^{0}=\left[\begin{array}{ccc}
0.137 & 0 & 0 \\
0 & 0.127 & 0.055 \\
0 & 0.055 & -0.186
\end{array}\right]
$$

The transformation stress can thus be obtained according to Eqn. 4-7 or as the following

$$
\left(\begin{array}{l}
\sigma_{11} \\
\sigma_{22} \\
\sigma_{33} \\
\sigma_{23} \\
\sigma_{13} \\
\sigma_{12}
\end{array}\right)=-\left[\begin{array}{cccccc}
C_{11} & C_{12} & C_{12} & 0 & 0 & 0 \\
C_{12} & C_{11} & C_{12} & 0 & 0 & 0 \\
C_{12} & C_{12} & C_{11} & 0 & 0 & 0 \\
0 & 0 & 0 & C_{44} & 0 & 0 \\
0 & 0 & 0 & 0 & C_{44} & 0 \\
0 & 0 & 0 & 0 & 0 & C_{44}
\end{array}\right]\left(\begin{array}{c}
\varepsilon_{11} \\
\varepsilon_{22} \\
\varepsilon_{33} \\
2 \varepsilon_{23} \\
2 \varepsilon_{13} \\
2 \varepsilon_{12}
\end{array}\right)
$$

And the results of $\sigma_{\mathrm{ij}}{ }^{0}$ for both $\mathrm{K}-\mathrm{S}$ and $\mathrm{N}-\mathrm{W}$ relationships are

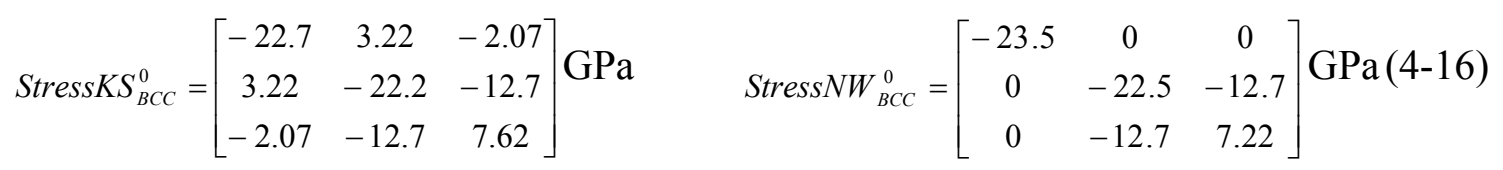

\section{Relaxed strain field}

The relaxed strain field is obtained by Eqn. 4-9 


$$
\varepsilon_{i j}(R)=-1 / 2\left\lfloor n_{i} \Omega_{j k}^{e}+n_{j} \Omega_{i k}^{e}\right\rfloor \sigma_{k l}^{0} n_{l} \theta(R)
$$

where the Green's elastic matrix $\Omega$ and transformation stress $\sigma^{0}$ have been calculated in Eqn. 4-13 and 4-15, respectively. The shape function $\theta(\boldsymbol{R})$ is 1 within the inclusion and 0 everywhere else in the matrix. The normal direction of the habit plane $\boldsymbol{n}$ is $(0,0.707$, 0.707). Therefore, the relaxed strain fields for the K-S and N-W relationships are

$$
\text { KS Relax }=\left[\begin{array}{ccc}
0 & -0.003 & -0.003 \\
-0.003 & 0.191 & 0.034 \\
-0.003 & 0.034 & -0.123
\end{array}\right] \quad \text { NW Relax }=\left[\begin{array}{ccc}
0 & 0 & 0 \\
0 & 0.191 & 0.034 \\
0 & 0.034 & -0.123
\end{array}\right]
$$

\section{Overall stress and strain in martensite}

Total elastic strain in martensite is the transformation strain minus the relaxed strain:

$$
\begin{aligned}
& \text { KSTotal }=\left[\begin{array}{ccc}
0.135 & -0.016 & 0.009 \\
-0.016 & 0.128 & 0.055 \\
0.009 & 0.055 & -0.187
\end{array}\right]-\left[\begin{array}{ccc}
0 & -0.003 & -0.003 \\
-0.003 & 0.191 & 0.034 \\
-0.003 & 0.034 & -0.123
\end{array}\right]=\left[\begin{array}{ccc}
0.135 & -0.013 & 0.013 \\
-0.013 & -0.063 & 0.021 \\
0.013 & 0.021 & -0.063
\end{array}\right] \\
& \text { NWTotal }=\left[\begin{array}{ccc}
0.136 & 0 & 0 \\
0 & 0.127 & 0.055 \\
0 & 0.055 & -0.187
\end{array}\right]-\left[\begin{array}{ccc}
0 & 0 & 0 \\
0 & 0.191 & 0.034 \\
0 & 0.034 & -0.123
\end{array}\right]=\left[\begin{array}{ccc}
0.136 & 0 & 0 \\
0 & -0.064 & 0.021 \\
0 & 0.021 & -0.064
\end{array}\right]
\end{aligned}
$$

We shall rotate the strain matrix above to a new coordinate system $[100],[0,1,-1]$ and [011] since [011] is the normal direction of habit plane.

$$
\begin{aligned}
& \text { KSTotal' }=\left[\begin{array}{ccc}
0.135 & -0.018 & -0.005 \\
-0.018 & -0.085 & 0.157 \\
-0.005 & 0.157 & 0.026
\end{array}\right]-\left[\begin{array}{ccc}
0 & 0 & -0.005 \\
0 & 0 & 0.157 \\
-0.005 & 0.157 & -0.123
\end{array}\right]=\left[\begin{array}{ccc}
0.135 & -0.018 & 0 \\
-0.018 & -0.085 & 0 \\
0 & 0 & -0.042
\end{array}\right] \\
& \text { NWTotal' }=\left[\begin{array}{ccc}
0.136 & 0 & 0 \\
0 & -0.085 & 0.157 \\
0 & 0.157 & 0.026
\end{array}\right]-\left[\begin{array}{ccc}
0 & 0 & 0 \\
0 & 0 & 0.157 \\
0 & 0.157 & 0.068
\end{array}\right]=\left[\begin{array}{ccc}
0.136 & 0 & 0 \\
0 & -0.085 & 0 \\
0 & 0 & -0.042
\end{array}\right]
\end{aligned}
$$

The final stress after relaxation can be calculated from the final relaxed strain in the original BCC system according to Eqn. 4-9: 
KS RelaxStress $=\left[\begin{array}{ccc}13.94 & -2.97 & 2.97 \\ -2.97 & -4.89 & 4.89 \\ 2.97 & 4.89 & 4.89\end{array}\right] G P a \quad N W$ RelaxStress $=\left[\begin{array}{ccc}14.15 & 0 & 0 \\ 0 & -4.86 & 4.86 \\ 0 & 4.86 & -4.86\end{array}\right] G P a$

After rotating it to the in-and-out (011) plane coordinate as we did for strain, we have

KS RelaxStress' $=\left[\begin{array}{ccc}13.94 & -4.21 & 0 \\ -4.21 & -9.78 & 0 \\ 0 & 0 & 0\end{array}\right] G P a \quad N W$ RelaxStress ${ }^{\prime}=\left[\begin{array}{ccc}14.15 & 0 & 0 \\ 0 & -9.72 & 0 \\ 0 & 0 & 0\end{array}\right] G P a$

In both cases, it is apparent that after the relaxation all strain and stress components outside the habit plane become zero. The strain in the through-thickness direction is even over-relaxed in order to compensate for the Poisson effect. However, the strain components within the habit plane remain unchanged, leading to a large in-plane stress state in both cases. This indicates that according to elastic theory, an infinite thin martensite plate will completely relax all elastic stress and strain in the through-thickness direction, but can not relax anything inside the habit plane at all.

\section{$\underline{4.3 .3 \quad \text { Necessity of invariant plane strain condition }}$}

The results from elastic theory calculation provide us with another reason why an invariant plane strain condition is necessary for martensitic transformation.

From the energy point of view, if the surface energy is ignored, the elastic strain energy will be the primary source of an energy barrier during a martensitic transformation. In general, the elastic energy can be calculated as following:

$$
E=1 / 2 * V^{*} \sigma_{i j}{ }^{*} \varepsilon_{i j}
$$


where the $\sigma_{i j}, \varepsilon_{i j}$ are the relaxed stress and strain in the final state calculated as in the previous section. For example, in the case of the K-S relationship in pure Fe, the strain energy barrier according to Eqn. 4-22 is about $2400 \mathrm{cal} / \mathrm{mol}$. If compared with the chemical free energy driving force of martensitic transformation at $0 \mathrm{~K}$, which, according to the estimates given by Kaufman and Cohen, is only $1200 \mathrm{cal} / \mathrm{mole},{ }^{[88,89]}$ then the elastic strain energy is too high (almost double) for the driving force to overcome. According to this analysis, therefore, it is almost impossible for the martensitic transformation to occur.

This conflict may result from on the fact that the in-plane stress components within the habit plane have not been relaxed at all. As shown in Eqn. 4-21, the elastic stress within the habit plane is so large $(\sim 14 \mathrm{GPa})$ that it is already approaching the ideal strength of iron. ${ }^{[28]}$ This elastic stress is entirely due to the large unrelaxed distortion caused by the transformation at the interface between the martensite and austenite. Thus, in order to decrease the elastic strain energy, an invariant plane strain condition is necessary to compensate the distortions and relax the elastic strain at the interface.

\subsubsection{Achievement of invariant strain condition}

Since an invariant plane strain is a necessary condition for martensitic transformation to start and proceed, we shall discuss the mechanisms on how to achieve this condition during the martensitic transformation. 
The first method to achieve an invariant plane strain condition is through dislocation slip. This mechanism has been observed in low alloy steel with dislocated lath martensitic microstructure. Using Wechsler-Liberman-Read theory, ${ }^{[77]}$ it is easy to show that a simple shear can be adopted for the complementary strain to create an invariant plane strain condition. As discussed in the last section, since the elastic stress is so high that it approaches the ideal strength of $\mathrm{Fe}$, it is reasonable to believe that some dislocations will be emitted or generated within the interfacial planes to compensate for the large unrelaxed distortion. In other words, self-energy associated with the dislocations will compensate for the elastic strain energy induced by the transformation.

The second method to achieve an invariant plane strain condition is mechanical twining, which has been observed in high alloy martensitic steel with internally twinned plates. The twinned regions have different K-S relations, such as KS1 and KS2 whose strain matrix has been described in Eqn. 4-2 and 4-3, In fact, W-L-R theory was first applied to twinned martensite in an FCC to BCC transformation. It has been shown that an invariant plane can exist only when the volume ratio of twinned regions has a certain value. ${ }^{[77]}$

The third circumstance under which an invariant plane strain condition will be created is when K-S and N-W relationships coexist in adjacent laths within the same packet (as observed in Chapter III). It is well known that neither K-S nor N-W alone satisfies the invariant plane strain condition since both are just a Bain distortion plus a rigid body rotation. Therefore, none of the principal distortions in either the K-S or N-W matrix are equal to unity. However, if both K-S and N-W relations co-exist in alternating laths of a 
sandwich structure, according to Eqn. 4-1 and 4-2, the macroscopic shape deformation associated with overall phase transformation can be described as:

$$
\operatorname{Def}_{\text {Toal }}=x \text { DefKS }+(1-x) \operatorname{DefN} W=x\left[\begin{array}{ccc}
0.789 & -0.194 & -0.074 \\
-0.134 & 1.117 & -0.074 \\
0.060 & 0.060 & 1.131
\end{array}\right]+(1-x)\left[\begin{array}{ccc}
1.126 & -0.008 & 0.096 \\
-0.008 & 1.126 & 0.096 \\
-0.136 & -0.136 & 0.791
\end{array}\right]
$$

where $\mathrm{x}$ is the volume fraction of the K-S related martensite crystal. In order to produce an invariant plane strain, one of the principal distortions of the overall deformation matrix has to be unity. To satisfy this condition, $\mathrm{x}$ is solved to be 0.60 so that the eigenvalues of the overall deformation matrix are $1.129,1,0.924$.

From the elastic strain point of view, both the K-S and N-W relationships are nothing but different variants of Bain strain if we ignore the small shear components (this will be discussed in detail in next section). Thus, the net transformation strain of a packet consisting of alternating K-S and $\mathrm{N}-\mathrm{W}$ relationships is:

$\varepsilon_{\text {toal }}=x$ Strain $K S+(1-x)$ Strain $N W=x\left[\begin{array}{ccc}-0.197 & 0 & 0 \\ 0 & 0.135 & 0 \\ 0 & 0 & 0.135\end{array}\right]+(1-x)\left[\begin{array}{ccc}0.134 & 0 & 0 \\ 0 & 0.134 & 0 \\ 0 & 0 & -0.198\end{array}\right]$

where $\mathrm{x}$ is again the volume fraction of the K-S related martensite within the packet. When $x=\varepsilon_{33} /\left(\varepsilon_{33}-\varepsilon_{11}\right)=0.6$, the net strain matrix has a dyadic form ${ }^{[90]}$ as:

$$
\varepsilon_{\text {total }}=\left[\begin{array}{ccc}
-0.065 & 0 & 0 \\
0 & 0.135 & 0 \\
0 & 0 & 0
\end{array}\right]
$$

Although it is difficult to obtain experimentally an accurate value of the volume fraction of austenite $\mathrm{x}$ getting, a rough estimation can be obtained from the area fraction in the 
dark field images taken in a TEM. Using this method, it is found that $\mathrm{x}$ is about $60 \% \sim 65 \%$, in good agreement with the calculated value.

It should be noted that this only works in the ideal case of an infinite thin plate in an infinite large parent matrix. In the real world, getting a dyadic transformation strain or an invariant plane strain at the interface will never completely eliminate the elastic strains. However, this is still considered the near-minimum elastic energy state when the system is a bulk material. 


\subsection{Crystallographic Relationship and Grain Refinement}

Since our ultimate goal is to refine the effective grain size, the neighboring subvolumes must have different orientations in order to generate large angle misorientation in between. In the case of high strength steel with lath martensitic microstructure, those subvolumes are packets, blocks or laths that have different orientation relationships with parent austenite. However, the question remains as to whether or not different orientation relationships between adjacent domains necessarily indicates two different "effective grains". We shall discuss this question in detail below.

\subsubsection{Grouping K-S and N-W relations into three Bain variant groups}

From transformation strain matrices in Eqn. 4-1, 4-2, and 4-3, it is apparent that the shear mechanisms that create K-S or N-W related martensite from parent austenite are almost identical to the Bain distortion if the small shear components are disregarded. Furthermore, we can call the compression along $\mathrm{x}$ axis Bain variant group $\mathrm{A}, \mathrm{B}$ for $\mathrm{y}$ and $\mathrm{C}$ for the $\mathrm{z}$ axis in the FCC lattice respectively. In this case, two twin-related K-S relationships and one N-W relationship (associated with a specific close-packed direction in one close-packed plane) belong to different Bain variant groups. As a consequence, we can also group all $24 \mathrm{~K}-\mathrm{S}$ and $12 \mathrm{~N}-\mathrm{W}$ relationhips into three Bain groups according to their elastic strain matrices during martensitic transformation as shown in Table 4-1 and 4-2. 
Table 4-1 Re-grouping 24 K-S orientation relations into three Bain variant groups

\begin{tabular}{|c|c|c|c|c|c|}
\hline O. R. Variant & FCC Plane & BCC Plane & FCC Direction & BCC Direction & Bain Variant \\
\hline \hline KS-1 & $(111)$ & $(011)$ & {$[-110]$} & {$[11-1]$} & $\mathrm{A}$ \\
\hline $\mathrm{KS}-2$ & $(111)$ & $(011)$ & {$[-110]$} & {$[-11-1]$} & $\mathrm{B}$ \\
\hline $\mathrm{KS}-3$ & $(111)$ & $(011)$ & {$[01-1]$} & {$[-11-1]$} & $\mathrm{C}$ \\
\hline $\mathrm{KS}-4$ & $(111)$ & $(011)$ & {$[01-1]$} & {$[11-1]$} & $\mathrm{B}$ \\
\hline $\mathrm{KS}-5$ & $(111)$ & $(011)$ & {$[10-1]$} & {$[11-1]$} & $\mathrm{C}$ \\
\hline $\mathrm{KS}-6$ & $(111)$ & $(011)$ & {$[10-1]$} & {$[-11-1]$} & $\mathrm{A}$ \\
\hline \hline $\mathrm{KS}-7$ & $(-111)$ & $(011)$ & {$[110]$} & {$[11-1]$} & $\mathrm{A}$ \\
\hline $\mathrm{KS}-8$ & $(-111)$ & $(011)$ & {$[110]$} & {$[-11-1]$} & $\mathrm{B}$ \\
\hline $\mathrm{KS}-9$ & $(-111)$ & $(011)$ & {$[01-1]$} & {$[-11-1]$} & $\mathrm{C}$ \\
\hline $\mathrm{KS}-10$ & $(-111)$ & $(011)$ & {$[01-1]$} & {$[11-1]$} & $\mathrm{B}$ \\
\hline $\mathrm{KS}-11$ & $(-111)$ & $(011)$ & {$[-10-1]$} & {$[11-1]$} & $\mathrm{C}$ \\
\hline $\mathrm{KS}-12$ & $(-111)$ & $(011)$ & {$[-10-1]$} & {$[-11-1]$} & $\mathrm{A}$ \\
\hline \hline $\mathrm{KS}-13$ & $(1-11)$ & $(011)$ & {$[110]$} & {$[11-1]$} & $\mathrm{B}$ \\
\hline $\mathrm{KS}-14$ & $(1-11)$ & $(011)$ & {$[110]$} & {$[-11-1]$} & $\mathrm{A}$ \\
\hline $\mathrm{KS}-15$ & $(1-11)$ & $(011)$ & {$[10-1]$} & {$[-11-1]$} & $\mathrm{C}$ \\
\hline $\mathrm{KS}-16$ & $(1-11)$ & $(011)$ & {$[10-1]$} & {$[11-1]$} & $\mathrm{A}$ \\
\hline $\mathrm{KS}-17$ & $(1-11)$ & $(011)$ & {$[0-1-1]$} & {$[11-1]$} & $\mathrm{C}$ \\
\hline $\mathrm{KS}-18$ & $(1-11)$ & $(011)$ & {$[0-1-1]$} & {$[-11-1]$} & $\mathrm{B}$ \\
\hline \hline $\mathrm{KS}-19$ & $(11-1)$ & $(011)$ & {$[-10-1]$} & {$[11-1]$} & $\mathrm{A}$ \\
\hline $\mathrm{KS}-20$ & $(11-1)$ & $(011)$ & {$[-10-1]$} & {$[-11-1]$} & $\mathrm{C}$ \\
\hline $\mathrm{KS}-21$ & $(11-1)$ & $(011)$ & {$[011]$} & {$[-11-1]$} & $\mathrm{B}$ \\
\hline $\mathrm{KS}-22$ & $(11-1)$ & $(011)$ & {$[011]$} & {$[11-1]$} & $\mathrm{C}$ \\
\hline $\mathrm{KS}-23$ & $(11-1)$ & $(011)$ & {$[1-10]$} & {$[11-1]$} & $\mathrm{B}$ \\
\hline $\mathrm{KS}-24$ & $(11-1)$ & $(011)$ & {$[1-10]$} & {$[-11-1]$} & $\mathrm{A}$ \\
\hline
\end{tabular}

Table 4-2 Re-grouping $12 \mathrm{~N}-\mathrm{W}$ orientation relations into three Bain variant groups

\begin{tabular}{|c|c|c|c|c|c|}
\hline O. R. Variant & FCC Plane & BCC Plane & FCC Direction & BCC Direction & Bain Variant \\
\hline$N W-1$ & (111) & $(011)$ & [1-10] & [100] & $\mathrm{C}$ \\
\hline$N W-2$ & (111) & (011) & [10-1] & [100] & B \\
\hline$N W-3$ & (111) & (011) & {$[0-11]$} & [100] & A \\
\hline$N W-4$ & $(-111)$ & $(011)$ & [110] & [100] & C \\
\hline$N W-5$ & $(-111)$ & $(011)$ & {$[01-1]$} & [100] & A \\
\hline NW-6 & $(-111)$ & (011) & [101] & [100] & B \\
\hline $\mathrm{NW}-7$ & $(1-11)$ & (011) & [110] & [100] & C \\
\hline$N W-8$ & $(1-11)$ & (011) & [011] & [100] & A \\
\hline$N W-9$ & $(1-11)$ & (011) & {$[-101]$} & [100] & $B$ \\
\hline NW-10 & $(11-1)$ & (011) & [101] & [100] & $B$ \\
\hline NW-11 & $(11-1)$ & (011) & {$[1-10]$} & [100] & C \\
\hline NW-12 & $(11-1)$ & (011) & [011] & [100] & $A$ \\
\hline
\end{tabular}




\subsubsection{Orientation relations between and within different Bain groups}

Since ferrous steel cleaves on $\{100\}$ planes, it is our ultimate goal to refine the size of subvolumes that have a large angle misorientation between their $\{100\}$ cleavage planes. Two different orientation relations in the adjacent subvolumes do not necessarily mean two different effective grains in terms of transgranular cleavage fracture. A complete stereographic projection of $\{100\}$ pole figures for all K-S and N-W related martensite is shown in Fig. 4-6 on the basis of the austenite FCC lattice. Clearly the $\{100\}$ pole figures of K-S and N-W relationships are clustered into three local regimes based on the Bain variant groups they are associated with. Only those from different Bain variant group will have a large angle misorientation in between. Within the same Bain variant group, the orientation relationships are related to each other by a small angle rigid body rotation. We shall examine this here in detail.

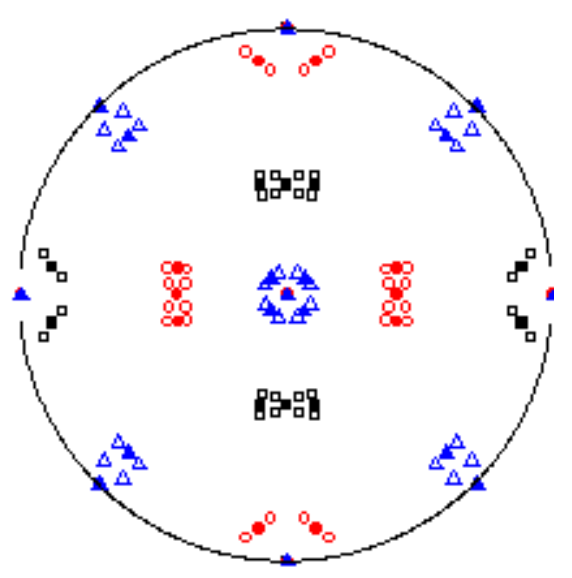

Fig. 4-6 Stereographic projection of $\{100\}$ pole figures for K-S and N-W related martensite in an austenite basis. Open and close marks represent K-S and N-W relationships respectively. Circles, squares, and triangles represent the relations from Bain variant group $\mathrm{A}, \mathrm{B}$, and C, respectively. ${ }^{[91]}$ 


\section{Orientation relations within a Bain variant group}

As discussed previously, Bain deformation will transform the compressed principal axis of FCC into one of the principle axes of BCC, say $[001]_{\gamma} / /[001]_{\alpha^{\prime}}$, and transform the other two elongated diagonal axes that are perpendicular to the first one into the other two principle axes of BCC, i.e., $[110]_{\gamma} / /[100]_{\alpha^{\prime}}$ and $[\overline{1} 10]_{\gamma} / /[010]_{\alpha^{\prime}}$. However, none of these correspondences will fully satisfy either the K-S or N-W orientation relationship. Thus, a rigid body rotation is needed. The transformation matrix can be expressed as $T=R \times B$ where $B$ is the Bain correspondence and $R$ is the rigid body rotation of $\theta$ degrees around the axis [uvw], and can be described in a general form ${ }^{[92]}$ as:

$$
R=\left[\begin{array}{ccc}
\left(1-\mathrm{u}^{2}\right) \operatorname{Cos} \theta+u^{2} & u v(1-\operatorname{Cos} \theta)+w \operatorname{Sin} \theta & u w(1-\operatorname{Cos} \theta)-v \operatorname{Sin} \theta \\
u v(1-\operatorname{Cos} \theta)-w \operatorname{Sin} \theta & \left(1-v^{2}\right) \operatorname{Cos} \theta+v^{2} & v w(1-\operatorname{Cos} \theta)+u \operatorname{Sin} \theta \\
u w(1-\operatorname{Cos} \theta)+v \operatorname{Sin} \theta & v w(1-\operatorname{Cos} \theta)-u \operatorname{Sin} \theta & \left(1-\mathrm{w}^{2}\right) \operatorname{Cos} \theta+w^{2}
\end{array}\right]
$$

For example, in the case of NW-3 that belongs to Bain variant group A according to

Table 4-2, the orientation relationship is $[0 \overline{1} 1]_{\gamma} / /[100]_{\alpha^{\prime}}$ and $(111)_{\gamma} / /(011)_{\alpha^{\prime}}$. After transformation of Bain variant $\mathrm{A}$, the correspondences are $[100]_{\gamma} / /[010]_{\alpha^{\prime}},[011]_{\gamma} / /[001]_{\alpha^{\prime}}$, $[0 \overline{1} 1]_{\gamma} / /[100]_{\alpha^{\prime}}$. Thus, the first part of the relationship is satisfied while the second is not. As a result, a rigid body rotation that will rotate $(111)_{\gamma}$ into $(011)_{\alpha^{\prime}}$ is required. In addition, this rotation is with respect to the rotation axis $[0-11]_{\gamma}$, or $[100]_{\alpha^{\prime}}$ since this crystal axis must remain unchanged during the rotation. In the trace of [0-11], (111) $\gamma$ now has a $54.7^{\circ}$ angle with $(011)_{\gamma}$ or $(001)_{\alpha^{\prime}}$ and $35.3^{\circ}$ with $(100)_{\gamma}$, or $(010)_{\alpha^{\prime}}$. In order to make it parallel to $(011)_{\alpha}$, these two angles have to be $45^{\circ}$. Therefore the rotation angle is $54.7^{\circ}-45^{\circ}=9.7^{\circ}$. Hence the Bain and rotation matrix can be expressed as: 
$\mathbf{B}=\left[\begin{array}{ccc}0 & -1 / \sqrt{2} & 1 / \sqrt{2} \\ 1 & 0 & 0 \\ 0 & 1 / \sqrt{2} & 1 / \sqrt{2}\end{array}\right], \quad R=\left[\begin{array}{ccc}1 & 0 & 0 \\ 0 & 0.986 & 0.168 \\ 0 & -0.168 & 0.986\end{array}\right]$

The transformation matrix is

$T_{N W 3}=R \bullet$ Bain $A=\left[\begin{array}{ccc}0 & -0.707 & 0.707 \\ 0.986 & 0.120 & 0.120 \\ -0.169 & 0.697 & 0.697\end{array}\right]$

This is indeed the NW-3 orientation relationship as it can be verified with $\mathrm{T} \times\{0.577$, $0.577,0.577\}=\{0,0.707,0.707\}, T \times\{0,-0.707,0.707\}=\{1,0,0\}$. Therefore, all of the NW relations can be considered a Bain deformation plus a rotation of angle $9.7^{\circ}$.

In the case of the K-S relationship, for example, KS-1, the orientation relationship is $(111)_{\gamma} / /(011)_{\alpha^{\prime}}$ and $[-110]_{\gamma} / /[11-1]_{\alpha^{\prime}}$. After Bain deformation A, none of these two relations are satisfied. Therefore, we can first rotate $(111)_{\gamma}$ to $(011)_{\alpha^{\prime}}$ just like in the case of the N-W relationship, and then rotate $[-110]_{\gamma}$ to $[11-1]_{\alpha^{\prime}}$ with respect to the rotation axis of $(111)_{\gamma}$ i.e. $(011)_{\alpha^{\prime}}$. The rotation angle in the second rotation is the angular difference between the K-S and N-W in the plane of $(111)_{\gamma}$, which is about $5.16^{\circ}$, as shown in Fig. 4-2. Hence, the Bain deformation matrix, rigid body rotation and transformation matrix can be determined as:

$$
\begin{aligned}
& \mathbf{B}=\left[\begin{array}{ccc}
0 & 1 / \sqrt{2} & -1 / \sqrt{2} \\
0 & 1 / \sqrt{2} & 1 / \sqrt{2} \\
1 & 0 & 0
\end{array}\right], R_{1}=\left[\begin{array}{ccc}
1 & 0 & 0 \\
0 & 0.986 & -0.168 \\
0 & 0.168 & 0.986
\end{array}\right], R_{2}=\left[\begin{array}{ccc}
0.996 & 0.064 & -0.064 \\
-0.064 & 0.998 & 0.002 \\
0.064 & 0.002 & 0.998
\end{array}\right] \\
& T_{k s 1}=R_{2} \bullet R_{1} \bullet \text { BainA }=\left[\begin{array}{ccc}
-0.075 & 0.742 & -0.667 \\
-0.167 & 0.650 & 0.742 \\
0.983 & 0.167 & 0.075
\end{array}\right]
\end{aligned}
$$


This transformation matrix can be verified as $\mathrm{T} \times\{0.577,0.577,0.577\}=\{0,0.707,0.707\}$; $\mathrm{T} \times\{-0.707,0.707,0\}=\{0.577,0.577,-0.577\}$, which satisfies the requirement of the KS-1 relationship.

We can also use one rigid body rotation to rotate both directions. The rotation matrix is:

$$
R=R_{2} \bullet R_{1}=\left[\begin{array}{ccc}
0.996 & 0.052 & -0.074 \\
-0.064 & 0.984 & -0.166 \\
0.064 & 0.167 & 0.984
\end{array}\right]
$$

This rotation corresponds to the rotation of $10.8^{\circ}$ with respect to the rotation axis of $[0.881,-0.362,-0.306]$ after the Bain Deformation.

Therefore, it is apparent that within one Bain variant group, the N-W relationship is just $9.7^{\circ}$ and the K-S relations are $10.8^{\circ}$ away from Bain relationship. Between the K-S and $\mathrm{N}-\mathrm{W}$ within the same Bain group, there is only a $5.16^{\circ}$ difference. In summary, there are only small angle misorientations between those orientation relationships within the same Bain variant group.

\section{Orientation relationships between different Bain variant groups}

From Fig. 4-6, it is clear that there is large angle misorientation between those relationships that belong to different Bain variant groups. It can also be understood more easily through the 2-D schematic illustration shown in Fig. 4-7. The square represents a 2-D lattice with adjacent structure domains sharing a (11) plane. After a cubic to tetragonal martensitic transformation with different Bain variants, a gap is formed between two domains and thus a rigid body rotation is needed in order to bring them back 
together and maintain the continuity of the lattice structure. As a consequence, a large angle misorientation of plane (10) is generated between the adjacent structure domains. This rotation would not be necessary if the adjacent domains undergo the transformation with the same Bain variant. Therefore, there is large angle misorientation of cleavage plane $\{100\}$ if adjacent structure domains undergo martensitic transformations that have orientation relationships from different Bain variant groups.
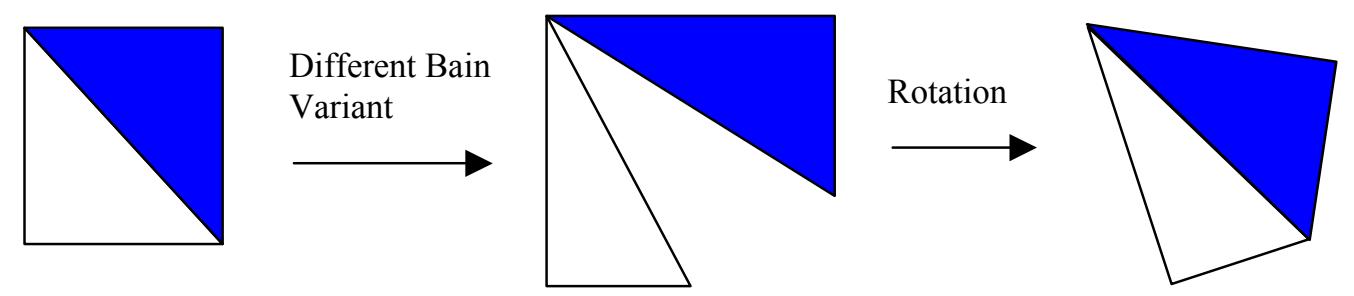

Fig. 4-7 Schematic illustration of formation of the large angle misorientation when the adjacent structure domains undergoes transformation with different Bain variants.

\subsubsection{Four-color theorem and grain refinement in ferrite}

As discovered in the last section, in terms of transgranular cleavage fracture, we really do not have all $24 \mathrm{~K}-\mathrm{S}$ and $12 \mathrm{~N}-\mathrm{W}$ orientation relationships, but rather three different Bain variants available to refine the effective grain size. Thus, the remaining question is whether these three Bain variants are enough to fulfill the task of grain refinement.

Before we try to answer this question, let us introduce the Four-Color Theorem first. The Four-Color Problem dated back to 1852 when Francis Guthrie, while trying to color the map of counties of England, noticed that four colors sufficed. The Four-Color Theorem states that any map can be colored using four colors in such a way that adjacent regions 
(i.e. those sharing at least a common boundary segment, not just a point) receive different colors as shown in Fig. 4-8. ${ }^{[93]}$

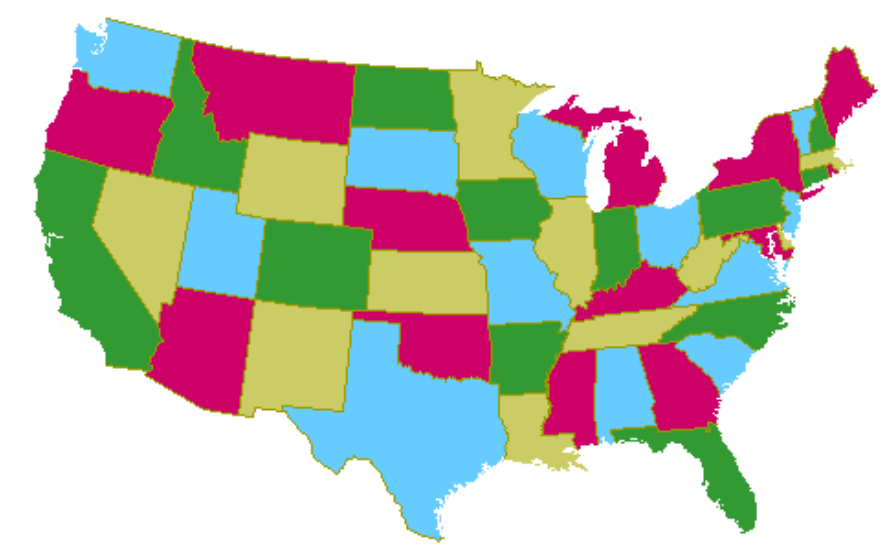

Fig. 4-8 An illustration of four-color theorem using US map.

Now let us imagine different Bain variant groups as different colors and try to map a 2-D randomly distributed pattern-- for example, several ferrite grains that are generated from one austenite grain. ${ }^{[91,94]}$ (It has been found that ferrite grains have some specific orientation relationships with the parent austenite even though they are transformed through nucleation and grain growth). From the four-color theorem, we know it is not enough to map this 2-D pattern with only three Bain variants (i.e. three different colors). As a consequence, some grains in a 2-D random pattern will share the same Bain variant (have same color) with its neighbors, leading to an easy cleavage facet that can go through two or more grains without being diverged. In the case of a 3-D pattern, even more colors are needed in order to fulfill the requirement. In fact, it has been well known that in a ferritic steel refined through thermomechanical processing the cleavage facet size generally is about two or three times larger than the grain size of ferrite. ${ }^{[95]}$ This phenomenon can be well explained by the four-color theorem and the fact that there are 
only three Bain variants available to create large angle misorientation between adjacent subvolumes. Therefore, it is impossible to completely refine the grain size in a randomly distributed pattern, such as that obtained in ferritic steel through thermomechanical processing.

However, things become very different in lath martensitic steel due to its special morphology. Individual martensitic laths have a roughly parallelepiped shape $(\mathrm{a}<\mathrm{b}<<\mathrm{c})$, and are stacked together with parallel boundaries in each packet. ${ }^{[96]}$ In this case, only two different Bain variants are needed to completely refine the effective grain size by alternating each lath with different Bain variants as in an "ABABAB..." stacking sequence. Therefore, three Bain variants are enough to achieve grain refinement in lath martensitic steel. 


\subsection{Elastic Theory and Thermal Mechanism of Grain Refinement}

In lath martensitic steel, the "effective" grain size in terms of transgranular cleavage fracture is the size of the subvolumes that have orientation relationships from different Bain groups needed in order to create a large angle misorientation of cleavage planes between the adjacent structure domains. Therefore, the question left to us is how to force the system to choose specific orientation relationships from different Bain groups in the adjacent subvolumes and how to refine the size of these subvolumes.

Linear elasticity theory shows that whether a system will choose one single orientation relationship or multiple variants over a certain range depends upon both the constraint and deformation condition. When the transformation solid is unstrained, the elastic strain induced by a transformation can be relaxed and the transformation proceeds through the growth of a single-variant plate in order to minimize the surface energy. On the other hand, if the transformation is constrained by its surroundings, the elastic strain can be relaxed, at least partially, by forming other variants from different Bain groups that can compensate the strain with each other. $\mathrm{Xu}$ and Morris found similar results using

computer simulations. ${ }^{[84,85]}$ Therefore, a system will at least want to choose orientation relationships from different Bain variant groups in the adjacent subvolumes if geometric constraints to martensitic transformation are present. In a real crystal, the transformation is constrained by the stable matrix phase, grain boundaries, or some internal defects that limit the growth of a single plate, and by the growth of the plates that nucleate separately and interfere with one another. 
We shall discuss three cases of constraints from large to small scale and the thermal mechanisms of grain refinement associated with them.

\subsubsection{Constrained by grain boundary}

In a homogeneous, defect-free polycrystal of austenite, grain boundaries will be the major constraint for martensitic transformation. In general, prior austenite grains will be subdivided into three or more packets, which usually have orientation relationships from different Bain variant groups in order to compensate for the elastic strain with one another (Fig. 4-9 (a)). It has been well known that if there are orientation relationships from all three Bain groups with relatively the same volume fraction, all of the shear strain will be cancelled out, leading to a transformation with no shape change but only dilatation. Within each packet, however, the whole prior austenite grain is constrained by its neighbors while the packets are not since their shape changes have already been compensated for by the adjacent packets in the same grain. Hence, once a variant nucleates, it will start to grow as it does in an unconstrained matrix. Then the second lath with the same variant will nucleate on the interface of the first lath in order to minimize the surface energy so that the single variant martensitic transformation can proceed until the whole packet is filled by those crystallographically well-aligned laths. This microstructure has been observed when alloys are quenched from high temperature since the dislocations have been annealed out and the alloy composition has been homogenized, such as quenching after a reverse austenization (Q) treatment or normalization $(\mathrm{N})$ treatment. 
When the major constraint is the prior austenite grain boundaries, the proper approach to refine the effective grain size, or in this case, the packet size, is to decrease the austenite grain size. The thermal treatment to accomplish this is austenite reversion, followed by a quenching to re-transform to martensite before the austenite can grow to an appreciable size. This is referred to as a "quench" or "Q" treatment, which is generally included in the processing of lath martensite steels. ${ }^{[50]}$

\subsubsection{Constrained by separately nucleated structure domains}

When there are defects present, such as dislocations, they will usually promote the nucleation of a martensitic transformation by relaxing the elastic strain in one way or another. Therefore, if more heterogeneous nucleation sites are introduced, it is possible to nucleate martensite laths at different locations within the same packet. These laths will grow and thicken as in an unconstrained matrix until they interfere with one another. As a result, a block-like microstructure will result when austenite is quenched with a high dislocation density as shown in Fig. 4-9 (b). The effective grain size in this case is equal to the size of the blocks, which is determined by the amount of nucleation sites.

There are two key issues when the constraint for martensitic transformation is the separately nucleated blocks. First, we need to introduce a high dislocation density, i.e., the austenite has to be heavily deformed. Secondly, the dislocations should still remain after heating to the reverse austenite temperature. The thermal treatment to accomplish

this is a "spark" $\mathrm{Q}$ heat treatment ${ }^{[97]}$ or a "step" Q treatment. ${ }^{[98]} \mathrm{A}$ "spark" $\mathrm{Q}$ treatment 
involves heating the alloy to the reverse austenite temperature for a very short period so that recrystallization has no time to occur. A "step" Q treatment involves heating the alloy at a temperature window above the reverse austenite temperature but below the recrystallization point to maintain the dislocation density. Both methods have been used for $\mathrm{Ni}$-steel in our research group.

\subsubsection{Constrained by stable matrix}

When there is a chemical inhomogeneity in the austenite matrix, different alloy element contents in the local structure domains will lead to different Ms temperatures. During quenching, martensitic transformation will proceed in the solute-lean constituent first. However, this transformation will soon be stopped once the frontiers of transformation meet the adjacent solute-rich subvolumes, where the chemical driving force is too small (because of the lower Ms temperature) to overcome the increasing resistance from the transformation strain energy. Once the temperature drops further, the martensitic transformation will resume in the solute-rich part. Having been heavily deformed by the previous transformation, and constrained by the surrounding martensite, the solute-rich austenite will pick up the orientation relationship from another Bain variant group to compensate for the overall elastic strain during the transformation (Fig. 4-9 (c)). As a consequence, the effective grain size is the size of the subvolumes that have different alloy composition with the adjacent ones.

The thermal treatment associated with this is an "LQ" treatment, or a combination of intercritical annealing followed by an austenite reversion treatment that has been 
performed in AerMet 100 as discussed in Chapter III. An intercritical annealing will form a lamellar structure with solute $(\mathrm{Ni}, \mathrm{C})$-rich fresh martensite and solute-lean tempered martensite in the alternating laths within the same packet. When heating to the austenite field during Q treatment, both constituents of the L-treated steel revert to austenite. If it is held in the $\gamma$-region for some time after reversion, the carbon atoms may redistribute, but the compositional inhomogeneity of solute $\mathrm{Ni}$ will be preserved due to the low diffusivity of the substitutional species in the close-packed FCC $\gamma$ phase. During the subsequent cooling, the dual-phase alloy undergoes a two-step martensitic transformation as described before. As a result, orientation relationships from different Bain variant groups will appear in the alternating laths, causing a large angle misorientation between adjacent laths. Therefore, the effective grain size is the size of the individual lath, which is in the range of submicron.

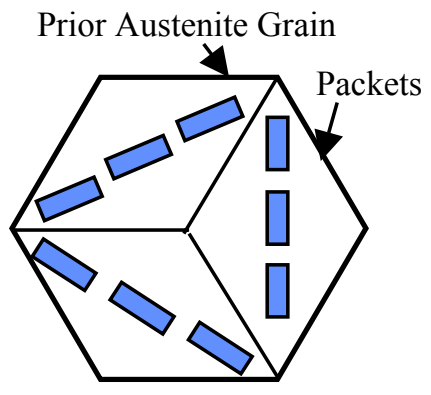

(a)

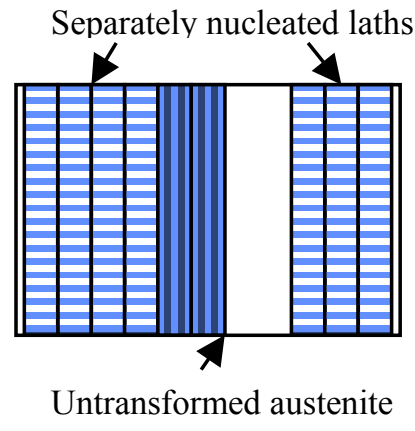

(b)

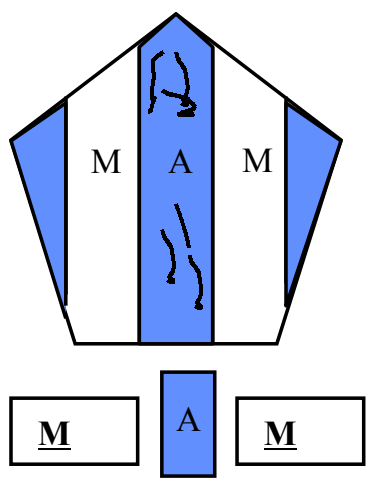

(c)

Fig. 4-9 Illustration of the effective grain size: (a) the packet size when constrained by prior austenite grain boundaries, (b) the block size when constrained by separately nucleated lath and (c) the individual laths when constrained by an untransformed matrix. 
It has to be pointed out that having a geometric constraint is only a necessary but not a sufficient condition for appearance of a multiple variant microstructure. When geometric constraint prevents the relaxation of elastic strain, the system can still relax through one of the two methods: either relax the strain plastically by deforming the matrix and generating a series of dislocations or relax it elastically by forming a multiple-variant microstructure in the adjacent subvolumes. The system will do whichever is more energetically favorable. In general, the former has been widely observed in dislocated martensitic steels. However, under some circumstances, the latter will be chosen if generating dislocations to relax the strain is too difficult. For example, in the case of a LQ treatment, the austenite has already been heavily deformed after the first step martensitic transformation. It will be very difficult to create more dislocations in an already workhardened matrix. This is especially true for austenite due to its very high work hardening coefficient. Thus, the system has to relax strain through the elastic method, i.e., choose multiple orientation relationships from different Bain groups. In summary, both appropriate deformation and constraint conditions are necessary for the appearance of multi-variant microstructure. 


\subsection{Revisiting the Meaning of Effective Grain Size and Grain Refinement}

All discussions in this chapter are referring to the effective grain size in terms of transgranular cleavage fracture. Therefore, the key issue is to disrupt the coherent length of crystallographically-preferred cleavage planes $\{100\}$. However, under some other circumstances, the effective grain size may have a different meaning. For example, if we have a hydrogen-assisted fracture, the prevalent fracture surface is the $\{110\}$ planes due

to the decohesion of lath boundaries. ${ }^{[99]}$ Therefore, the effective grain size for hydrogen embrittlement is the size of the structure domains that share the same lath boundary plane $\{110\}$, rather than the $\{100\}$ planes. These two measures of grain size are not necessarily the same, and do not necessarily respond in the same way when the steel is processed. ${ }^{[49,99]}$ Moreover, $\{110\}$ planes are also the preferred planes for dislocation glide. Hence, the effective grain size in terms of plastic deformation or yielding strength is the coherent length along these planes. The thermal treatment that can refine the effective grain size of cleavage fracture may not necessarily refine the effective grain size for strength. In fact, if we choose two orientation relationships from different Bain variant groups, although there are large angle misorientations between their $\{100\}$ planes as shown in Fig. 4-8, there are not large angle misorientations between their $\{110\}$ planes. As shown in Fig. 410 , there generally are only slight misorientations among $\{110\}$ planes for any orientation relationship, no matter which Bain groups they belong to. As a consequence, the thermal mechanisms discussed before that will refine the effective grain size for cleavage fracture by introducing orientation relationship from different Bain groups will 
have very little effect on the effective grain size in terms of yield strength and hydrogen embrittlement.

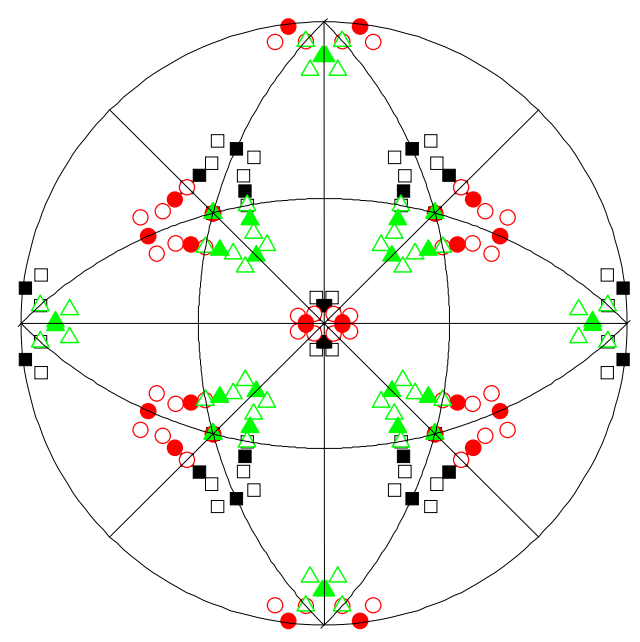

Fig. 4-10 Stereographic projection of $\{110\}$ pole figures for K-S and N-W related martensite in an austenite basis. All solid labels represent $\mathrm{N}-\mathrm{W}$ relationships while open ones represent K-S relationships. The squares, circles, and triangles represent the relationships belonging to Bain variant group A, B and C, respectively. ${ }^{[91]}$ 


\section{$\underline{4.7 \quad \text { Conclusions }}$}

The transformation strains associated with K-S and N-W orientation relationships have been calculated. Based on these calculations, the following conclusions have been drawn:

1. Introducing an invariant plane strain condition is a necessary requirement considering the elastic stress and energy induced during the martensitic transformation. There are three ways to accomplish this. Besides dislocation slip and mechanical twining which were discovered before, the coexistence of K-S and N-W relationships can also accomplish this condition.

2. Twenty-four K-S orientation relationships and twelve N-W relationships can be divided into three Bain variant groups based on the crystallographic theory and elastic strain calculations. Only those relationships from different Bain groups will create large angle misorientation between adjacent subvolumes leading to refinement of the effective grain size. Those from the same Bain group will relate to each other through a small angle rigid body rotation.

3. If the geometric constraint prevents the relaxation of elastic strain induced by transformation, relations from different Bain variant groups will be chosen in the adjacent structure domains in order to compensate for the induced strains. Therefore, introducing constraints to martensitic transformation on different levels through corresponding thermal treatments is an effective way to refine the effective grain size 
of lath martensitic steel. Different constraints will produce different effective grain sizes, such as packets, blocks, and individual laths.

4. Different mechanisms may have different meanings for the effective grain size. As a consequence, the thermal mechanism that will refine the effective grain size for cleavage fracture may have very little effect on refining the effective grain size for strength or hydrogen embrittlement. 
$<$ Appendix $>$

\section{$\underline{\text { Transformation of Interlath Austenite during Transgranular }}$}

\section{Fracture in Lath Martensitic Steel}

It is generally accepted that thermally stable interlath austenite can be beneficial to the toughness of lath martensitic steel at cryogenic temperatures. ${ }^{[100]}$ A series of thermal treatments have been developed accordingly on Ni-based ferritic steel for cryogenic service. For example, after intercritical tempering (L) for about $1 \mathrm{hr}$ at $600^{\circ} \mathrm{C}$, $9 \mathrm{Ni}$ steel exhibits a remarkable suppression of its ductile-to-brittle transition temperature (DBTT) for more than $100^{\circ} \mathrm{C}$ by precipitating austenite along lath boundaries with a dense distribution. Although this method has been widely used with commercial success for many years, the fundamental reason for its effectiveness remains an important and sometimes controversial topic of research. Early ideas that the soft austenite phase served to blunt a propagating brittle crack have been ruled out by the observation that all austenite transforms to martensite in front of a crack tip. ${ }^{[1,52]}$ It has also been suggested that the austenite serves as an "interstitial scavenger" and hereby promotes a cleaner and more ductile martensite. However, such theory requires large amounts of transforming austenite, which is generally not observed in an L-treated $9 \mathrm{Ni}$ steel. ${ }^{[56]}$

A transmission electron microscopy (TEM) study of Ni steel by Morris, et al. has showed that thermally stable interlath austenite will transform to those crystallographic variants that are different from the common orientation relationship in the neighboring laths. ${ }^{[53]}$ As shown in Fig. A-1, the orientation relationship of fresh martensite transformed under 
mechanical load from precipitated austenite is in fact the N-W relationship while the surrounding lath in the same packet has a K-S relationship with the parent austenite. This suggests that these variants are most compatible with the applied stress since the transformation is under mechanical loading. Thus, co-operative crack propagation along common cleavage planes in a packet of aligned martensite laths is disturbed by introducing these new variants of martensite. As a result, this impairment promotes more ductile-dimple fracture and displays better cryogenic toughness. In this appendix, based on linear elasticity theory, we will discuss why this is more favorable energetically than growing with the same variant as its surrounding laths.

As shown in the diffraction pattern in Fig. A-1, tempered martensite and fresh martensite transformed under mechanical loading have K-S and N-W relationships with the retained interlath austenite, specifically an orientation relationship of as $(111)_{\gamma} / /(011)_{\alpha^{\prime}}{ }^{\mathrm{K}-\mathrm{S}} / /(011)_{\alpha^{\prime}}{ }^{\mathrm{N}}$ and $[\overline{1} 10]_{\gamma} / /[11 \overline{1}]_{\alpha^{\prime}}^{K-S} / /[\overline{1} 00]_{\alpha^{\prime}}^{N}$. Therefore, the lath boundary is a close-packed plane in both FCC and BCC. Because of the special morphology of martensite lath (Length $>>$ Width >> Thickness), a propagating transgranular crack will pick the cleavage plane (001) or (010) to approach the lath boundary (011) plane in order to avoid the length direction and minimize the surface energy. Without losing generality, we can further assume that the cleavage plane is (001) and that the crack propagates along [010] direction. 


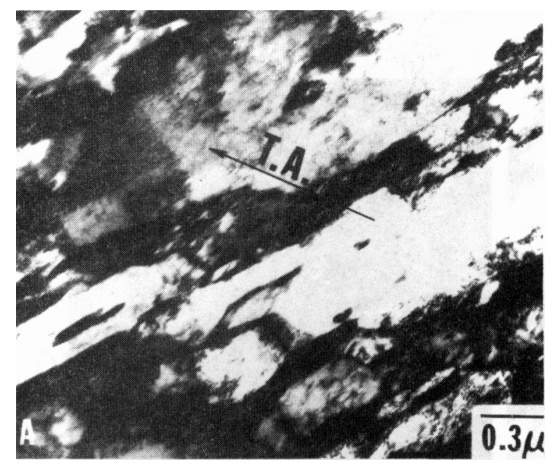

(a)

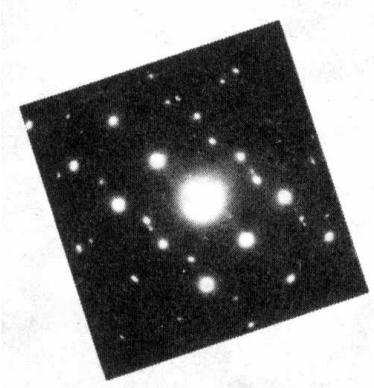

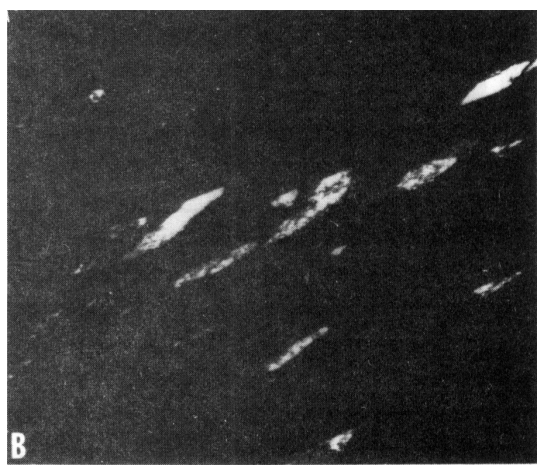

(b)

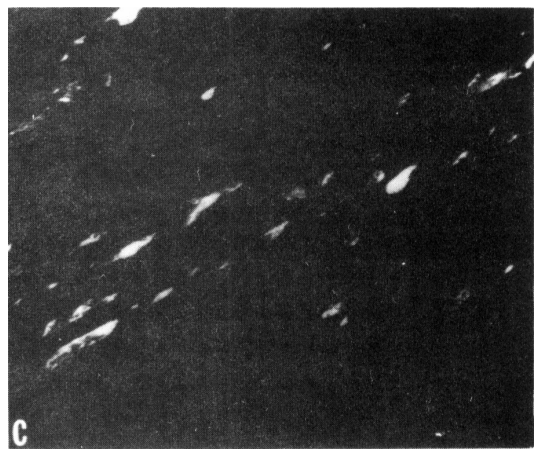

(c)

Fig. A-1 TEM micrographs of a 9Ni steel after mechanical loading: (a) bright field, (b) dark field of mechanically transformed martensite and (c) dark field of the retained austenite which still remains. ${ }^{[50]}$

As shown in Fig. A-2, if we ignore the thickness of the interlath austenite, which usually is very thin, the distance between the austenite and the crack tip can be expressed as

$$
r=r_{0} / \cos (\pi / 4-\theta)
$$

where $r_{0}$ is the closest distance at $45^{\circ}$. The stress state ahead of the crack tip can be assumed as a plane strain condition since the width of the lath is much larger than thickness. According to linear elastic fracture mechanics, the stress field ahead of crack tip can be described as: ${ }^{[7]}$ 


$$
\begin{aligned}
& \sigma_{x x}=\frac{K_{I}}{\sqrt{2 \pi r}} \cos \left(\frac{\theta}{2}\right)\left[1-\sin \left(\frac{\theta}{2}\right) \sin \left(\frac{3 \theta}{2}\right)\right] \\
& \sigma_{y y}=\frac{K_{I}}{\sqrt{2 \pi r}} \cos \left(\frac{\theta}{2}\right)\left[1+\sin \left(\frac{\theta}{2}\right) \sin \left(\frac{3 \theta}{2}\right)\right] \\
& \tau_{x y}=\frac{K_{I}}{\sqrt{2 \pi r}} \cos \left(\frac{\theta}{2}\right) \sin \left(\frac{\theta}{2}\right) \cos \left(\frac{3 \theta}{2}\right) \\
& \sigma_{z z}=v\left(\sigma_{x x}+\sigma_{y y}\right)
\end{aligned}
$$

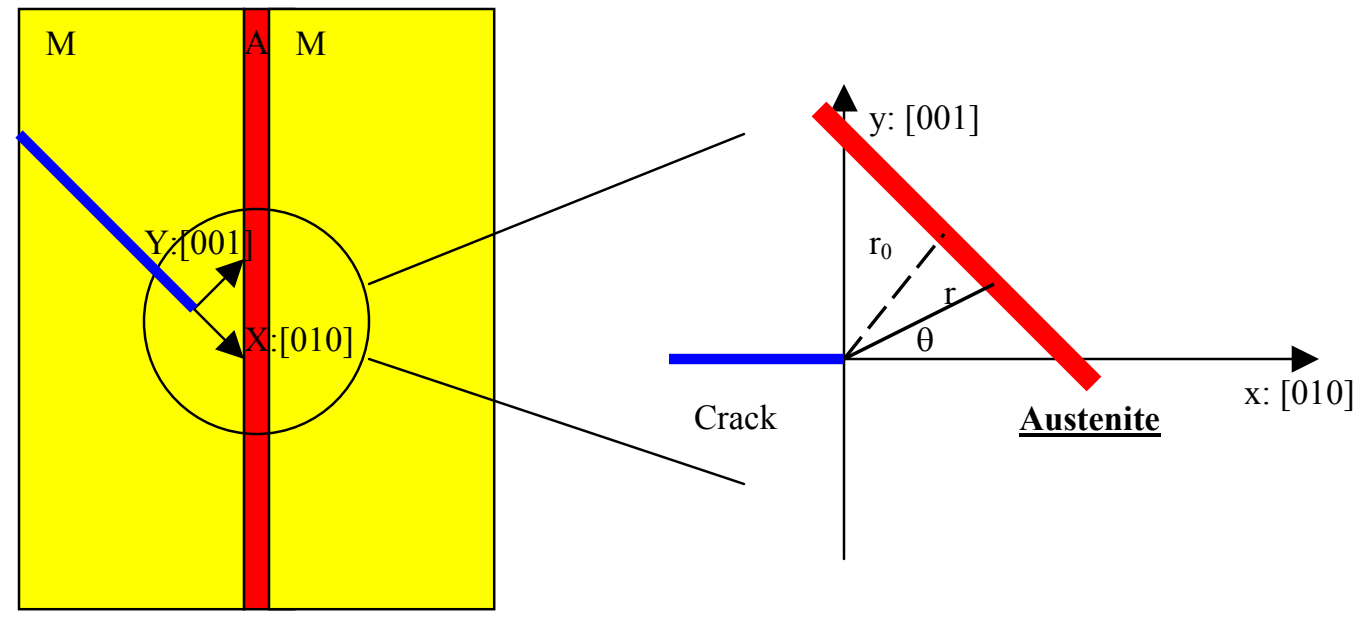

Fig. A-2 Schematic illustrations of retained austenite ahead of crack propagating along [001] cleavage plane of its nearby martensite.

Therefore the stress field in the martensite BCC coordinate can be written by substituting Eqn. A-1 into the stress field of Eqn. 2a-2c as following

$$
\sigma_{\mathbf{B}}=\left[\begin{array}{ccc}
\sigma_{z z} & 0 & 0 \\
0 & \sigma_{x x} & \tau_{x y} \\
0 & \tau_{x y} & \sigma_{y y}
\end{array}\right]
$$

This stress state can be transformed into the FCC coordinates of austenite since the martensite is related with it through a K-S relationship. The transformation between two 
coordinate systems can be obtained if the expressions of three non-coplanar vectors in both coordinates are known. In this case, $\mathrm{V}_{1}=0.577[1,1,1]_{\mathrm{F}}=0.707[0,1,1]_{\mathrm{B}} ; \mathrm{V}_{2}=0.707[-$ $1,1,0]_{\mathrm{F}}=0.577[-1,-1,1]_{\mathrm{B}} ; \mathrm{V}_{3}=0.621[-0.120,1.192,-1.072]_{\mathrm{F}}=[-1,0,0]_{\mathrm{B}}$ as obtained in previous chapter. Thus,

$$
R_{B \rightarrow F}^{K-S}=\left[\begin{array}{lll}
V_{11}^{\prime} & V_{12}^{\prime} & V_{13}^{\prime} \\
V_{21}^{\prime} & V_{22}^{\prime} & V_{23}^{\prime} \\
V_{31}^{\prime} & V_{32}^{\prime} & V_{33}^{\prime}
\end{array}\right]^{-1}\left[\begin{array}{lll}
V_{11} & V_{12} & V_{13} \\
V_{21} & V_{22} & V_{23} \\
V_{31} & V_{32} & V_{33}
\end{array}\right]=\left[\begin{array}{ccc}
0.074 & 0.983 & -0.166 \\
-0.742 & 0.167 & 0.650 \\
0.668 & 0.075 & 0.741
\end{array}\right]
$$

Therefore, the stress field in FCC coordinates of austenite is:

$$
\sigma_{F}=R_{B \rightarrow F} \bullet \sigma_{B} \bullet R_{B \rightarrow F}^{T}
$$

After knowing the stress field ahead of an approaching crack, what we want to investigate next is which variant the retained austenite will choose when it transforms to martensite under this external stress field. Since this is a transformation under external loading, the Gibbs free energy will be the criterion in thermodynamics to judge whether it picks up the same variant as its neighboring lath or a different one, for example, N-W relationship as shown in Fig. A-1. Hence, we have:

$$
G=G_{0}-V \sigma \otimes \varepsilon=G_{0}-V \sum_{i, j} \sigma_{i j} \varepsilon_{i j}
$$

where $\mathrm{G}_{0}$ is the energy difference between austenite and martensite phase, $\mathrm{V}$ is the volume of retained austenite under transformation, $\sigma$ is the external stress filed and $\varepsilon$ is the elastic strain induced by transformation, which, for both K-S and N-W relationships, have been calculated in Chapter IV as shown below.

$$
N W S=\left[\begin{array}{ccc}
0.137 & -0.0006 & 0 \\
-0.0006 & 0.137 & 0.0002 \\
0 & 0.0002 & -0.196
\end{array}\right] \quad K S S=\left[\begin{array}{ccc}
-0.196 & -0.0006 & 0 \\
-0.0006 & 0.137 & 0.0002 \\
0 & 0.0002 & 0.137
\end{array}\right]
$$


Therefore, the elastic energy term $(-\mathrm{V} \sigma \varepsilon)$ is plotted as a function of $\theta$ from 0 to $\pi / 2$ for both K-S and N-W related transformation as shown in Fig. A-3, indicating that at all locations (of the $\theta$ range), interlath retained austenite will transform to $\mathrm{N}-\mathrm{W}$ related martensite with much lower elastic energy and thus the total Gibbs free energy is less than that found when transforming to the same K-S variant as its neighboring laths.

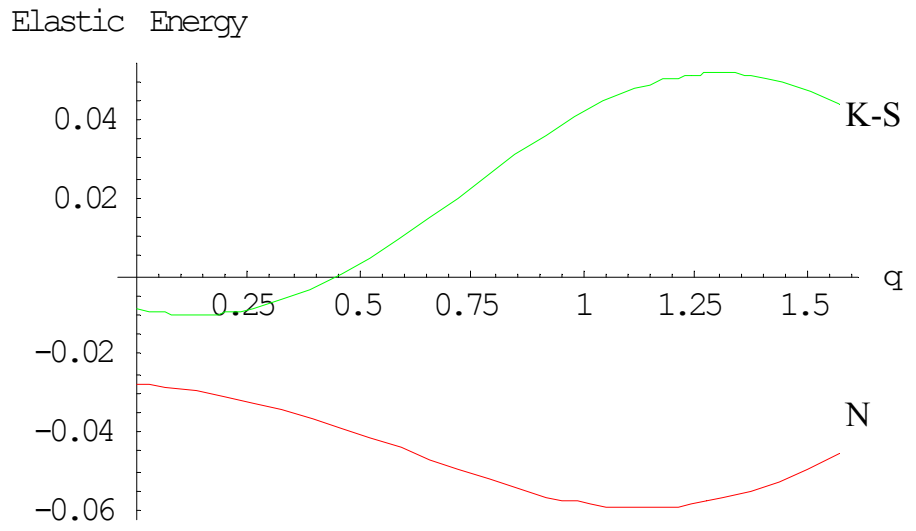

Fig. A-3 Elastic energy of transformation under external stress field vs. $\theta$ angle, showing that at every location the retained austenite will transform to $\mathrm{N}-\mathrm{W}$ related martensite rather than continuing the same K-S variant as its adjacent lath.

The crystallographic reason for this is actually very simple. No matter whether the crack is approaching along the $(001)$ or the $(010)$ cleavage plane, the two normal directions will always have a large tensile stress serving to open the crack according to linear elastic fracture mechanics. However, in the K-S related martensite lath, these two directions are in fact one in compression and one in tension, according to the Bain deformation in martensitic transformation (see Chapter IV). Therefore, if the retained austenite transforms to the same variant as its neighbor, it is energetically not favorable since one of these two directions has to be compressed although both are under high tensile stress. 
Therefore, the system would rather pick up another variant, in fact, a relationship from another Bain variant group in order to lower the elastic strain energy. In this case, it is a $\mathrm{N}-\mathrm{W}$ relationship that shares the same close-packed plane and close-packed direction of FCC as its adjacent K-S related martensite lath. In some other cases, twin-related K-S relationships have also been observed. ${ }^{[50]}$ Therefore, this is not an issue of $\mathrm{K}-\mathrm{S}$ or N-W relationships, but of Bain deformation. When a crack is propagating in a martensite lath, the retained austenite ahead of the crack tip will not continue the same variant in the nearby lath, nor those variants from the same Bain group. Instead, it will choose an orientation relationship from different Bain variant groups that can lower the total strain energy under the external stress field ahead of the crack tip.

In summary, the retained austenite along the lath boundaries ahead of a propagating crack will always transform to martensite with an orientation relationship from a different Bain variant group than that of its neighboring lath because of the external stress field in front of the crack tip. Thus, the common cleavage plane within a given packet will be disrupted by a different variant. Therefore, it will promote a more ductile-dimple fracture and demonstrate a better cryogenic toughness. 


\section{Reference}

1 A. Cottrell, Int. J. Pressure Vessels Piping, Vol. 64, 1995, pp171

2 V. F. Zackey, E. R. Parker, G. Thomas and J. W. Morris, Jr.: Mat. Sci. Eng., Vol. $16,1974, \mathrm{pp} 201$

3 J. W. Morris, Jr., Z. Guo, C. R. Krenn and Y.-H. Kim: ISIJ International, Vol. 41, 2001, pp599

$4 \quad$ J. R. Davis (ed.): ASM Special Handbook: Carbon and Alloy Steel, 1996 pp17

5 T. Uehara: Ph. D. Dissertation, University of Kyoto, Kyoto, Japan, 1998

6 K. Sato: Ph. D. Dissertation, University of California, Berkeley, 1998

7 T. L. Anderson: in Fracture Mechanics: Fundamentals and Applications, $2^{\text {nd }}$ edition, CRC Press, 1994

$8 \quad$ J. W. Morris, Jr.: MRS Symposium Proceedings, Vol. 539, 1999, pp23

$9 \quad$ S. K. Hwang and J. W. Morris, Jr.: Metall. Trans. A Vol. 11A, 1980, pp1197

10 M. Strum and J, W, Morris, Jr.: Adv. Cryo. Eng. (Materials), Vol. 34, 1987, pp371

11 J. W. Morris, Jr.: Earl R. Parker Symposium on Structure/Property Relationship, TMS Meeting, New Orleans, LA, March $3^{\text {rd }}, 1986$

12 T. M. Maccagno and J. F. Knott: Eng. Fracture Mech., Vol. 2/3, 1991, pp111

13 H. Ma: Int. J. Fracture, Vol. 89, 1998, pp143

14 J. F. Knott: in Fundamentals of Fracture Mechanics, Butterworths, London, 1973

15 R. O. Ritchie, B. Francis, and W. L. Server: Metall. Trans. A, Vol. 7, 1976, pp832

16 J. F. Knott: J. Iron Steel Inst., Vol. 204, 1966, pp104

17 J. W. Morris, Jr., Z, Guo and C. R. Krenn: Heat Treating: Steel Heat Treating in the New Millennium, Midea and Pfaffmann (eds.), ASM, 2000, pp526 
18 R. O. Ritchie, J. F. Knott and J. R. Rice: J. Mech. Phys. Solids, Vol. 21, 1973, pp395

19 R. M. McMeeking and D. M. Parks: ASTM STP 668, American Society of Testing and Materials, Philadelphia, 1979, pp175

20 J. F. Knott: Fracture, Vol. 1, ICF4, Waterloo Canada, 1977 pp61

21 B. L. Holian, R. Blumenfeld and P. Gumbsch: Phys. Rev. Letters, Vol. 78, 1997, pp78

22 P. Sumbsch and R. M. Cannon: MRS Bulletin, May, 2000, pp15

23 D. Roundy, C. R. Krenn, M. L. Cohen and J. M. Morris, Jr.: Philos. Mag. A, Vol. 81, 2001, pp 1725

24 J. W. Morris, Jr., C. R. Krenn, D. Roundy and M. L. Cohen: Mater. Sci. Eng. A, Vol. 310A, 2001, pp121

25 J. W. Morris, Jr. C. R. Krenn: Philos. Mag. A, Vol. 80, 2000, pp2827

26 J. Frenkel: Z. Phys., Vol. 37, 1926, pp572

27 E. Orowan: Rept. Prog. Phys., Vol. 12, 1949, pp185

28 D. Clatterbuck, D. C. Chrzan and J. W. Morris, Jr.: Phil. Mag. Letter, In Press

29 I. M. Mikhailovskii, P. Y. Poltinin and L. I. Fedorova: Sov. Phys. Solid State, Vol. $23,1981, \operatorname{pp} 757$

30 M. F. Doerner and W. D. Nix: J. Mater. Res., Vol. 1 1986, pp601

31 A. Hartmaier and P. Gumbsch: Journal of Computer-Aided Materials Design, Vol. 6, 1999, pp145

32 D. Frakas: MRS Bulletin, May 2000, pp35

33 F. R. N. Nabarro: Mater. Sci. Eng. A, Vol. 234, 1997, pp67 
34 H. M. Ledbetter and R. P. Reed: J. Phys. Chem. Ref. Data, Vol. 3, 1973, pp561

35 D. Clatterbuck: Unpublished Research, University of California Berkeley, 2001

36 K. J. Bowman, P.Miller, Z. Guo, R. Roeder, and K. P. Trumble: THERMEC'97. (Vol.2), Wollongong, NSW, Australia, July 1997. pp1169

37 E. O. Hall: Proc. Phys. Soc., B64, 1951, pp727

38 N. J. Petch: JISI, Vol. 174, 1953, pp25

39 A. H. Cottrell: Trans. AIME, Vol. 212, 1958, pp192

40 N. J. Petch: Phil. Mag., Vol. 3, 1958, pp1089

41 A. A. Griffith: Proc. Int. Congr. Appl. Mech., Vol. 55, 1924

42 N. S. Stoloff: Fracture, Vol. 6 Edited by H. Liebowitz, Academic Press, 1969, pp7

43 W. Y. Choo, POSCO Research Laboratories, Pohang, Korea, Private Communication, 2001

44 Kotobu Nagai, National Research Institute for Metals, Tsukuba, Japan, Private Communication, 2000

45 Z. Guo and J. W. Morris, Jr., Unpublished Research, UC Berkeley, 2000

46 J. W. Morris, Jr., J. I. Kim and C. K. Syn: in Advances in Metal Processing, Burke, Mehrabian and Weiss (eds.), Plenum Press, 1981, pp73

47 J. W. Morris, Jr.: in Mechanical Properties and Phase Transformations in Engineering Materials, Antolovich, Ritchie and Gerberich (eds.), TMS, Warrendale, PA, 1986, pp99

48 H. J. Kim, Y. H. Kim and J. W. Morris, Jr.: ISIJ International, Vol. 38, 1998, pp1277

49 Y. H. Kim, Ph. D. Dissertation, University of California, Berkeley, 1984 
Trans., Vol. 16A, 1985, pp2251

J. I. Kim, C. K. Syn and J. W. Morris, Jr.: Metall. Trans., Vol. 14A, 1983, pp83

C. K. Syn, S. Jin and J. W. Morris, Jr.: Metall. Trans., Vol. 7A, 1976, pp1827

B. Fultz and J. W. Morris, Jr.: Metall. Trans., Vol. 16A, 1985, pp2251

B. Fultz, J. I. Kim, Y. H. Kim and J. W. Morris, Jr.: Metall. Trans., Vol. 17A, pp967

57 J. I. Kim and J. W. Morris, Jr.: Metall. Trans., Vol. 12A, 1981, pp1957

58 J. I. Kim and J. W. Morris, Jr.: Proc. International Cryogenic Materials Conference, Kobe, Japan, Butterworth England, 1982, pp343

J. I. Kim, H. J. Kim and J. W. Morris, Jr.: Metall. Trans., Vol. 15A, 1984, pp2213

60 Z. Guo, K. Sato, T.-K. Lee and J.W. Morris, Jr.: in Ultrafine Grained Materials, Mishra, Semiatin, Suryanarayana, and Thadhanis, (eds.), TMS, Warrendale, Pa., 2000, pp51-62

61 Z. Guo, T. K. Lee and J. W. Morris, Jr.: Acta. Metall. Sinica, in press

62 Carpenter Technology, Inc. Data Sheet

63 M. Lacoude and C. Goux: C. R. Groupe 7, Vol. 259, 1964, pp1856

64 M. Lacoude and C. Goux: C. R. Groupe 7, Vol. 259, 1964, pp1117

65 Z. Nishiyama: Sci. Rep. Tohoku Univ., Vol. 23, 1934, pp637

66 S. Jana and C. M. Wayman: Trans. AIME, Vol. 239, 1967, pp1187 
67 K.-E. Thelning: in Steels and Its Heat Treatment, $2^{\text {nd }}$ Ed. Butterworths, London, 1984

68 D. A. Porter and K. E. Easterling: in Phase Transformation in Metals and Alloys, $2^{\text {nd }}$ Ed. Chapman \& Hall, London, 1992

69 G. Kurdjumov and G. Sachs: Z. Phys. Vol. 64, 1930, pp325

70 Z. Nishiyama: Sci. Rep. Tohoku Univ., Vol. 23, 1934, pp637

71 G. Wassermann: Mitt. K-W-I Eisenforsch. Vol. 17, 1935, pp149

72 Z. Nishiyama: in Martensitic Transformation, Academic Press, Inc., New York, 1978

73 M. Cohen, G. B. Olson and P. C. Clapp: Proceeding of International Conference on Martensitic Transformations (ICOMAT-79), Cambridge, MA, 1979, pp1

74 E. C. Bain: Trans. AIME., Vol. 70, 1924, pp25

75 C. M. Wayman: in Introduction to the Crystallography of the Martensitic Transformations, The MacMillian Company, New York, 1964

76 J. S. Bowles and J. K. Mackenzie: Acta. Metall., 1954, pp. 129

77 M. S. Wechsler, D. S. Lieberman, and T. A. Read: Trans. AIME J. Metals., Vol. 197, 1953, pp1503

78 J. W. Christian: in Theory of Transformation in Metals and Alloys, $2^{\text {nd }}$ Ed. Pergomon Press, New York, NY, 1975

79 J. D. Eshelby: Proc. Roy. Soc. A., Vol. 241, 1957, pp376

80 J. D. Eshelby: Proc. Roy. Soc. A., Vol. 252, 1959, pp561

81 A. G. Khachaturyan: Soviet Physics - Solid Sate, Vol. 8, 1967, pp2163 
82 A. G. Khachaturyan: Theory of Structural Transformation in Solids, Wiley, New York, NY, 1983

83 S. Wen, A. G. Khachaturyan, J. W. Morris, Jr. Proceeding of International Conference on Martensitic Transformations (ICOMAT-79), MIT, MA, 1979, pp94

84 P. Xu and J. W. Morris: Metall. Trans. Vol. 23A, 1992, pp2999

85 P. Xu and J. W. Morris: Metall. Trans. Vol. 24A, 1993, pp1281

86 P. Xu and J. W. Morris: Metall. Trans. Vol. 27A, 1996, pp1187

87 R. P. Reed and R. E. Schramm: J. Appl. Phys., Vol. 40, 1960, pp3453

88 L. Kaufman and M. Cohen: Trans. AIME, Vol. 206, 1956, pp1393

89 E. A. Owen, and Y. H. Liu: J. Iron Steel Inst., Vol. 163, 1949, pp132

90 P. Xu: Ph. D. Dissertation, University of California, Berkeley, 1993

91 C. S. Lee: POSCO Research Laboratory, Internal Report, 2001

92 K. Hoffman and R. Kunze: in Linear Algebra, $2^{\text {nd }}$ Ed. Prentice-Hall, Inc. Englewood Cliffs, New Jersey, 1971

93 T. L. Saaty and P. C. Cainen: in The Four-Color Problem: Assaults and Conquest, McGraw-Hill International Book Co., New York, 1977

94 H. Y. Yasuda, T. Sakata and Y. Umakoshi: Acta Mater., Vol. 47, 1999, pp1923

95 K.-K. Um, J.-K. Choi and W. Y. Choo: Technical Report, POSCO Technical Research Laboratories, Pohang, Korea, 2001

96 Sarikaya, Ph. D. Dissertation, University of California, Berkeley, 1982

97 Kobe Steel, Ltd., Welding Electrode Division, Tech. Report No. RDPD-7902

98 H. J. Kim and J. W. Morris, Jr.: Weld J., Vol. 62, 1983, pp210

99 Y. H. Kim, H. J. Kim and J. W. Morris, Jr.: Metall. Trans., Vol. 17A, 1986, pp1157 
100 C. W. Marshall, R. H. Heheman and A. R. Troiano: Trans. ASM, Vol. 55, 1962, pp135 